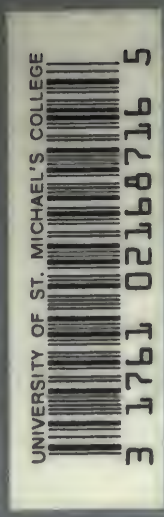

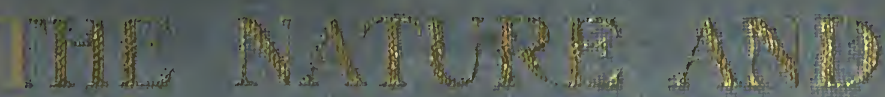

ORIOHN TOR KA:

Fand If WAMTho 


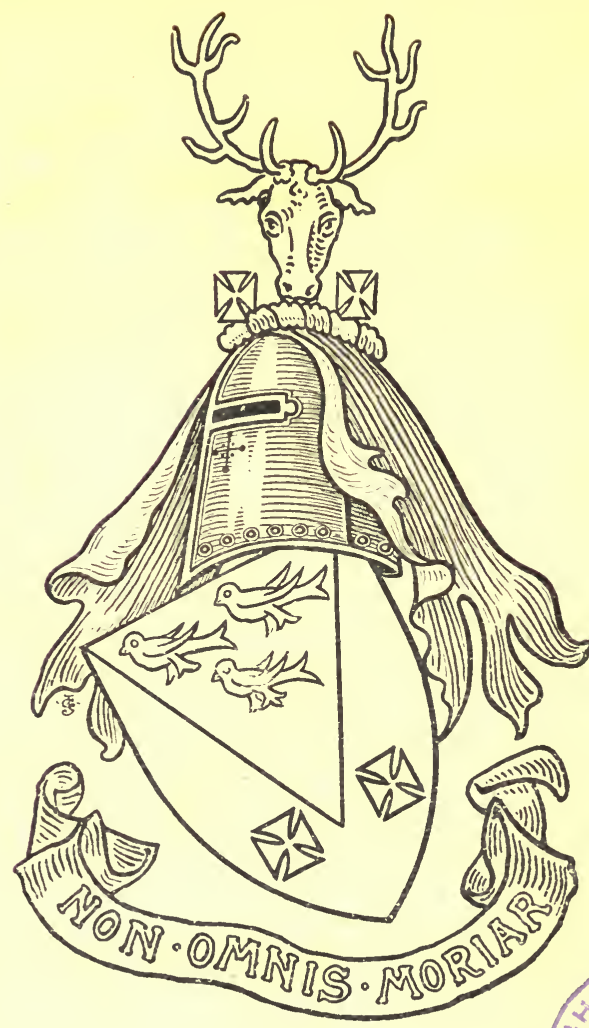

EX LIBRIS.

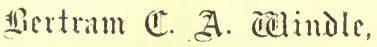

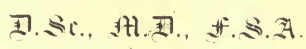


A TTEMPTS to explain the nature of life; to define Athe meaning of that term; to distinguish between living and non-living matter; of all these we have no lack, and the work on The Origin and Nature of Life, by Felix le Dantec (London: Hodder and Stoughton. I907), adds another to the series. We cannot say that the author leaves us any nearer a settlement of the question than we were before, but this at least can be said that he presents us with a very closely reasoned and interesting exposition of the subject from the standpoint of the confirmed and resolute mechanician. He has no doubt whatsoever that the effective synthesis which will produce living protoplasm in the laboratory will some day take place. When it does, "it will have no surprises in it, and it will be utterly useless. With the new knowledge acquired by science, the enlightened mind no longer needs to see the fabrication of protoplasm in order to be convinced of the absence of all essential difference and all absolute discontinuity between living and non-living matter" (p. 250). "This reads rather curiously from the same pen as a statement that a living being is not a machine made to accomplish one kind of work and that only, and that "circumstances so vary around any given animal, and the animal itself changes so quickly, that we may say without exaggeration, an animal never does twice the same thing in the whole course of its existence" (p. 67). If there is to be this constant change of method, one would conclude that there must be something to preside over and direct the changes, and one looks with interest to discover what that something is. It appears that we are to seek it in changes in the environment, including in that term the chemical characters and the changes of equilibrium in the colloids of the body itself. The author's argument, which is exceecingly imgenious, and as far as we are aware quite original, is very largely based upon the results obtained by the new science of serumtherapy, which we owe for the most part to the genius and discoveries of Pasteur, in whose Institute, if we are not mistaken, M. le Dantec has been a skilled and notable worker.

The author agrees with Lamarck that " the function creates the organ" (p. 74); but he assigns a meaning to the term organ which is perhaps wider than-certainly different to-the meaning which Lamarck would have attached to it. "' The definition of organ must be physiological, and the only possible definition is the following: 
An organ is the sum of the parts of an individual working together in the execution of a function " (p. 72). Thus it is inaccurate to speak of the liver as an organ. It is the liver plus those vascular, nervous and other parts of the body which co-operate with it in carrying out its function.

Now if a sheep be infected with virulent an thrax bacilli, the result will almost inevitably be its death, or we may say that the bacilli will assimilate the sheep. But let the sheep be first treated with an attenuated serum and then inoculated with the virulent bacilli. Then the circumstances of the case will be wholly altered, for the sheep will assimilate the bacilli. In le Dantec's phrase the sheep will have acquired an organ capable of destroying the bacilli, an organ not possessed by other unprotected sheep, and not possessed by itself until it had received the protective inoculations. Mainly on the lines of this class of observation the author builds up the argument which we have outlined, and maintains that there is no such thing as irritability as commonly understood, for "living bodies are inert just like others, that is, they are incapable of changing by themselves the state of repose or movement" (p. 158). Irritability of all kinds is the result of tactisms, whether we can recognize those tactisms or not.

After this nothing remains of the pretended spontaneity of movement in living bodies. An observer conversant with the results of all these experiments in tactisms knows that the movements he observes in living bodies through the microscope are due to the colloid and chemical reactions of the mobile beings and the medium.-p. 163.

We must content ourselves with indicating the line taken by the author in his book and by noting one or two points in connection with it. And first we may say that a great deal of the writer's work depends upon the changes in and behaviour of the colloids of the body, and that of colloid bodies in general, and of those of the human body in particular, we know, as the writer himself admits, as yet practically nothing. Hence a good deal of what he says may have to be very seriously modified in the future when these puzzling bodies have been more fully studied and are better understood.

Apart from this, however, we find ourselves wholly unconvinced by the argument of the book, namely, that the body is not only a mechanism, but that it is a fatally determined mechanism, and we are glad to find that in this conclusion we are joined by Professor Duncan, the 
The Jew Knowledge Series

Edited by Professor

ROBERT KENNEDY DUNCAN

editor of the scries, himself the author of an interesting work on the New Knowledge. In his introductory preface he agrees, as we must all do, that the living organism is a mechanism; " but there is a certain demonstration that the book does not contain, and that is, that because the living organism is a mechanism it is necessarily an automaton" (p. 7).

If life [continues Prof. Kennedy] is supposed to be a transcendental entity entering into or transacting the chemical processes, causing them in the sense of using energy, then there is no evidence that such an entity exists. But if on the contrary life is supposed to be a transcendental entity that is associated with the body, and in some measure interacts with it and guides it without interfer-

\section{THE NATURE AND ORIGIN OF LIFE}

ence (and that is by no means inconceivable since recent physics has plunged the ultimate nature of matter into such mystery) then there seems nothing in Professor Le Dantec's book to make unreant sonable a belief in the supposition,- - p. viii.

We must be allowed to dissent utterly from the writer's view that embryology is only the histology of very young beings (p. I I), for in our opinion such a statement is as inaccurate as would be the statement that history and geography were the same thing.

We must also protest against the issuing of a book such as this is, crammed with arguments and facts, without any trace of an index. Such an action is nothing short of an putrage on the reading public. _. B.C.A.W. 
Digitized by the Internet Archive in 2007 with funding from Microsoft Corporation 


\title{
THE NATURE AND ORIGIN OF LIFE
}

In the Light of New

Knowledge

\author{
By \\ FELIX LE DANTEC \\ Professor of the Faculty of Sciences at the Sorbonne
}

AN INTRODUCTORY PREFACE BY

R O B E T K. D U N C N

Autbor of "The Nerw Knowledge"

HODDER AND STOUGHTON

LONDON MCMVII 
Translated from the French by Stoddard Dewey

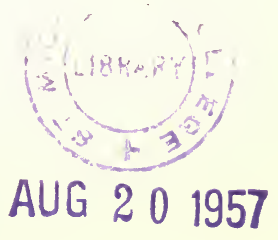

Butler and Tanner, The Selwood Printing Works, Frome, and London 
TO

TH. RIBOT

PROFESSOR AT THE COLLÉGE DE FRANCE DIRECTOR OF THE "REVUE PHILOSOPHIQUE" WITH REVERENCE AND AFFECTION

FROM HIS OLD FELLOW-WORKER

FELIX LE DANTEC

TY PLAD EN PLEUMEUR BODOU

June 17, 1906. 
" The notion that all the kinds of animals and plants may have come into existence by the growth and modification of primordial germs is as old as speculative thought; but the modern scientific form of the doctrine can be traced historically to the influence of several converging lines of philosophical speculation and of physical observation, none of which go farther back than the seventeenth century. These are :-

“1. The enunciation by Descartes of the conception that the physical universe, whether living or not living, is a mechanism, and that, as such, it is explicable on physical principles. . . ."

Huxley, Science and Culture, p. 290 ("The

Evolution of the Sum of Living Beings") 


\section{Editor's Preface}

Professor Felix Le Dantec, of the University of Paris, stands as perhaps the foremost champion of the mechanical theory of life, and in this book, written especially for the people of our speech, he has given us, in the simplicity of its expression, the clarity of its statement, and the keen logic of it, perhaps the best exposition of this subject extant. The conclusions that he arrives at seem coercive. The body is a mechanism, which in substance, energy, form and movements, proceeds absolutely in accordance with the laws of substance. In the demonstrations contained within this book, Professor Le Dantec has done great service in hastening what will be the inevitable conclusion of science.

But there is a certain demonstration that the book does not contain, and that is, that because the living organism is a mechanism it is necessarily an automaton.

In beginning his exposition of the phenomena of living matter by first casting out all consideration of phenomena "like that which I feel passing in myself" Professor Le Dantec permits himself to assume that the body is not only a mechanism (as he demonstrates so clearly) but that it is a fatally determined mechanism, a wholly different matter.

But the fact is that there are other things existing, perceptions, ideas, volitions and feelings, and while they do not necessarily enter into consideration in the proof that the 
living organism is a mass of matter acting wholly in the causal sequence, they must be reckoned with before they can be pronounced of no avail in the body's conduct. If life is supposed to be a transcendental entity entering into or transacting the chemical processes, causing them in the sense of using energy, then there is no evidence that such an entity exists. But if on the contrary life is supposed to be a transcendental entity that is associated with the body, and in some measure interacts with it and guides it without interference (and that is by no means inconceivable since recent physics has plunged the ultimate nature of matter into such mystery) then there seems nothing in Professor Le Dantec's book to make unreasonable a belief in the supposition.

Robert Kennedy Duncan.

University of Kansas,

1906. 


\title{
Contents
}

\section{BOOK FIRST-METHODS}

\author{
PART I
}

THE OBJECTIVE STUDY OF NATURAL BODIES

CHAPTER I

PAGE

The Principle of Continutty in Living and Not-Living Matter . . . . . . . . . . . 3

CHAPTER II

UntTy ANd Diversity In Living Bodies . . . . 7

\section{CHAPTER III}

Natural Phenomena Group themselves in Paraluel Series of Different Scales of Magnitude .

Examples : Vibratory, Oscillatory Movements-Chemistry, Colloids, Nebulae

CHAPTER IV

The Dimension of Life or the Place of Life in Nature

CHAPTER V 


\section{PART II}

\section{ANALYSIS OF NATURAL PHENOMENA}

CHAPTER VI

Dangers of too Hasty Analysis

\section{CHAPTER VII}

Artifictal and Natural Methods . . . . 34

Examples: Analyses of Audition-of Chemical Compound into Elements or Functions - of Bodies by means of our Sense OrgansMethod of Approximate Laws in Physies

\section{PART III}

FIRST METHOD OF ANALYSIS OF VITAL PHENOMENA-THE APPROXIMATE LAW OF ASSIMILATION

\section{CHAPTER VIII}

Artifictal Analysis as in the Physical Sctences

Chemical Equation of Elementary Life as Manifested-Destruction and Variation

\section{CHAPTER IX}

Useful Result of such Analysis in giving Life its Place among other Natural Phenomena

\section{CHAPTER $\mathrm{X}$}

Definition of Elementary Life in Chemical Language 
Various Conditions in WhICH a Living Body May Exist from the Chemical Point of View . . . . 61 Reserve Substances

$$
\text { PART IV }
$$

SECOND METHOD OF ANALYSIS: DECOMPOSITION INTO FUNCTIONS-STRICT LAW OF FUNCTIONAL ASSIMILATION

\section{CHAPTER XII}

Artificial Functions and Functions rationally defined

Organ and Function

\section{CHAPTER XIII}

Life is the Result of Struggle between Two Factors •

\section{CHAPTER XIV}

(Xeneral Applications of the Natural Method of Analysis

\section{CHAPTER XV}

Analysis by Means of Reagents of the same Dimension as LIFE

\section{CHAPTER XVI}

Protoplasmic Functions or Functions of Colloid MechanISM

Sheep Anthrax-Vaccination-Dead Colloids, Toxins, Foods 
Cases in which the Results of Functional Analysis are TRANSPORTABLE OUT OF THE BODY-SERUMTHERAPY • 95

Diastases, Excrementitial Substances: Physical Assimilation or Digestion

\section{CHAPTER XVIII}

Colloid States and Physiological Division of Labour $\quad 104$

CHAPTER XIX

Functions of the Mechanism as a Whole . . . 110

CHAPTER XX

Functional Assmilation a General Biological Law • 117

$$
\text { PART V }
$$

CONCORDANCE OF RESULTS OBTAINED BY THE TWO METHODS-AGREEMENT OF DARWIN'S SYSTEM WITH THAT OF LAMARCK

\section{CHAPTER XXI}

Natural Selection

\section{CHAPTER XXII}

Equilibrium and Habit . 


\title{
PART VI
}

\section{THIRD POINT OF VIEW-CONSERVATION OF ENERGY}

\author{
CHAPTER XXIII PAGE
}

The Study of Life and the Conservation of EnergyNew Proof that Life is a Phenomenon subject to the LAws of Mechanics $\quad . \quad$. $\quad . \quad$. 141

\section{BOOK SECOND—FACTS}

\section{PART VII}

PHENOMENA OF LIFE COMPARED WITH PHENOMENA OF NOT-LIVING MATTER

CHAPTER XXIV

Morphology of the Cell ANd Its Movements $\quad . \quad$. $\quad 149$

CHAPTER XXV

General Plan of Multicellular Beings . . 154

\section{CHAPTER XXVI}

Spontaneous Movement . 


\title{
CHAPTER XXVIII
}

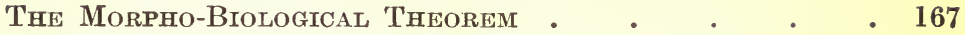

Merotomy-Morphogenic Function, importance of Colloid State

\section{CHAPTER XXIX}

Conduction Phenomena in Living and Not-Living Bodies

The Nervous System-Sense Organs, Sensorial Surfaces-Nerve Centres-Trophic Rôle of Nerves

\section{PART VIII}

\section{EVOLUTION IN LIVING AND NOT-LIVING MATTER}

\author{
CHAPTER XXX \\ Heredity in the Wide Sense and Individual Evolution

\section{CHAPTER XXXI}

Heredity of Acquired Characters a . . 204

Rudimentary Organs-Denial of Heredity of Acquired Characters 


\section{PART IX}

\section{BIPOLARITY IN LIVING AND NOT-LIVING MATTER}

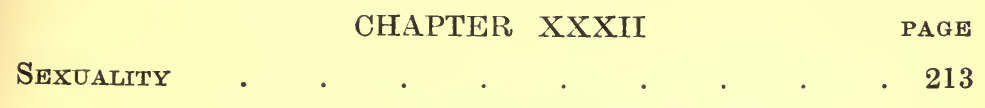

Artificial Parthenogenesis-Fecundation

\section{CHAPTER XXXIII}

Alternating Generation . . . . . . 221

CHAPTER XXXIV

Karyokinesis, or Indrect Cellular Division a . 224

\section{CHAPTER XXXV}

Hereditary Patrimonies in Fecundation

Secondary Sexual Characters

$$
\text { PART X }
$$

FORMATION OF SPECIES AND APPEARANCE OF LIFE

\section{CHAPTER XXXVI}

Continuous and Discontinuous Evolution . . . 233

Mutations

CHAPTER XXXVII

The Specres . . . . . 239 


\section{CHAPTER XXXVIII}

Genealogy of Present-Day Species

Fritz Müller's Principle

\section{CHAPTER XXXIX}

PAGE

243

Appearance of Life 


\title{
BOOK FIRST-METHODS
}

\author{
PART I \\ The Objective Study of Natural Bodies
}




\section{CHAPTER I}

\section{THE PRINCIPLE OF CONTINUITY IN LIVING AND NOT-LIVING MATTER}

BIology studies those characters which are common to all living beings whether animal or vegetable, and which, taken together, are uanting in not-living bodies. If we define life by taking the sum total of these common characters, it is evident that we ought never to find all of the characters in any not-living body. Otherwise our definition would be worthless.

Living beings have to be recognized among other natural bodies by this sum total of characters defining life, just as alcohols are known from other chemical bodies by their possessing all the characters which define the function alcohol. But this is not a reason why the differences which separate living bodies from not-living bodies should be more important than those which distinguish alcohols from aldehydes or amines. It may seem childish to insist on so evident a truth; but it is not useless because of the persistence, if not in science, at least in current language and literature, of old mystic ideas which preceded the advent of the scientific period of history and humanity.

When the study of living beings is carried out in a scientific manner, that is, by registering all their objective properties, we do not need to remember that we are alive ourselves; and we arrive at an objective definition of life as 
solid as that of the function alcohol or the standard metre. If, on the contrary, instead of employing this objective method, we define living bodies à priori as " bodies in which something passes like that which I feel passing in myself," we run up against assertions that are unverifiable and we do not obtain a scientific definition. This is what our ancestors did. Thanks to so defective a method, they were led to establish between living and not-living bodies a line of demarcation that could not be passed over and to consider life as something beyond the reach of experimental study.

Their method was bad because they really began, without suspecting it, by defining life from its objective characters; indeed it is only by objective characters that we can know whether bodies are living or not-living. Forgetting this first definition, which they used without suspecting it, they went on to give a second almost as unconsciously, defining a living body as we have said - " a body in which something passes like that which every one feels in himself." Such a definition is open to no further verification; and there is nothing in particular to show how it can be compared with that first and necessary objective definition if we are to be able to say that a body is living at all. Nevertheless they applied the second definition to bodies already defined by the first.

This is the origin of the anthropomorphic error, which locates a human mental quality in all bodies considered to be living; and one of the consequences is the belief that an abyss separates living from not-living bodies.

Learned men who were anxious to destroy so erroneous an idea tried to bridge over the abyss thus opened by anthropomorphists between living and not-living bodies; but they too kept to an à priori definition of life and came to the conclusion that life thus defined is universal-some- 
thing utterly unverifiable because the definition which they kept was purely subjective. Once more, a definition of life ought to separate living bodies from not-living bodies just as chemists separate alcohols from aldehydes. Such a result we obtain by limiting ourselves in our definition of life to the study of the objective characters of living beings. Now if we should accept a definition of life that can be applied to not-living bodies, the definition would be bad; it would not reach the end proposed; it would be like a definition of alcohols which could be applied to acetones.

The complete objective study of life is possible by the methods of the ordinary sciences of observation and experiment ; and to this must be limited our enunciation of the principle of continuity. It professes nothing more than this-between life and death the difference is of the same order as that which exists between a phenol and a sulphate, or between an electrified body and a neutral body. In other words, all phenomena which we study objectively in living beings can be analysed by the methods of physics and chemistry. In other words, again, life is not freed from the laws of universal mechanics.

If men had limited themselves from the beginning to objective methods of investigation, the question of the principle of continuity would never even have been raised. But an error of method has started up the problem and so it is of use to call attention to the following point. Although we define life by a sum total of characters, and consequently each body that possesses this sum total of characters is to be called living, other natural bodies without being living may also possess one or even several of these characters. Thus we may establish a classification of not-living bodies, in which we place nearest to living bodies those which possess a great number of characters in common with them (the enzymes, 
for example) and farthest away those which have but a small number of these characters. This is a new and more concrete and instructive form of the principle of continuity. It will help us to understand that living bodies may have come from not-living bodies by way of evolution.

When such objective study has been furnished-and only then-men may remember that they are themselves living beings; and they will verify the fact that the objective definition of life applies to themselves and to their fellows as well as to dogs, foxes and chestnut-trees. Then, verifying that they are also conscious, they may ask themselves whether other living beings are not so as well; they may seek to find out how it is that beings can be conscious and, for their mental satisfaction, they may imagine unverifiable hypotheses like that of universal consciousness. This is the hypothesis which has been mistakenly brought forward under the name of universal life. But, once more, if life is not to be defined so as to distinguish living bodies from not-living bodies, a man from his corpse, the word life will no longer have any meaning.

Moreover, if we are able to make a complete objective study of living beings without paying any attention whatever to this hypothesis of universal consciousness, it must be for the reason that such an hypothesis answers to nothing that appears objectively. The fact of being conscious does not intervene in the slightest degree in directing vital movements. Maudsley first and Huxley after him call this "the theory of epiphenomenal consciousness." Here we have only to limit ourselves to the purely objective study of living beings. 


\section{CHAPTER II}

\section{UNITY AND DIVERSITY IN LIVING BODIES}

Living bodies are interesting to the man of science from two points of view which, at first sight, seem contradictory : they strike us by their marvellous diversity; they astonish us by their remarkable unity.

Among scholars who choose to dwell in admiration on the differences whici: separate species, some-descriptive naturalists-aim either at the knowledge of forms and their classification (Descriptive Zoology and Botany) or at the comparison of forms with each other (Comparative Anatomy).

Others, considering each living being as a mechanism by itself, study the conditions and details of the mechanism's working (Physiology and Pathology of a living species) or apply themselves to bringing together the workings of the various specific mechanisms (Comparative Physiology and Pathology).

A last kind of these special researches attends to the genesis of a form or specific mechanism, either in the description of its development beginning with the egg (Embryology of a living species) or in the comparison of the phenomena of such development in different species (Comparative Embryology).

By joining the study of species of the present day with that of the fossil remains of species which have disappeared 
(Paleontology), we succeed in establishing between the two certain ties of relationship and descent; this is the special science of the Origin of Species.

The mere existence of "comparative" sciences (comparative anatomy, physiology, and embryology) along with the corresponding "descriptive" sciences is enough to prove that the diversity of living forms is not accompanied by absolute differences among the species. It allows us to constitute, in our general catalogue of beings, more or less extensive groups; and, among the various members of each of these groups, anatomical, physiological, or embryological comparison may be fruitful. Thus it is useful to compare, from the descriptive point of view, the dog, the horse, and the rabbit, which are mammals ; again it is interesting to compare the dog, cock, lizard, and trout, which are vertebrates ; while it would be fanciful to compare the anatomy of the rabbit and that of the sea urchin or earthworm. The latter have nothing in common; and the differences in anatomy become more strongly emphasized, if possible, when we pass from such animals to the chestnut-tree, the lily, and the mushroom, which are vegetable.

Yet man long since learned to unite under the common title of living beings bodies so little resembling each other as the dog, earthworm, fern and rosebush. Such bodies therefore must not only have something in common, but this something in common must be easy to find, since it did not escape men as ignorant as our ancestors. But we shall see that the common opinions regarding life are, to a certain degree, different from those which science establishes by means of instruments of research more powerful than those natural to man.

It is Biology which studies the characters common to all living beings, whether animal or vegetable-characters 
which, taken together, are wanting in not-living matter. $A l l$ these characters taken together ought to make up a definition of life, since it is their presence or absence which leads us to pronounce a body living or not; and if the definition is well made it ought to permit of a strict classification of all natural bodies-a classification in which no ambiguity should remain, so that a given body would fall necessarily under one or other of the defined categories, living or not-living. The comparative sciences of which we have already spoken (comparative anatomy, physiology, embryology), in the long run as living beings further and further remote are compared together, ought to permit of the building up of a Biology. For this it is enough to find in each living being something which remains common to all in the progressive extension of the groups we study, until we come to one group that comprises in itself the sum total of characters common to both the animal and vegetable kingdoms.

Now, with the old definition of the word "anatomy" it was evident that comparative anatomy could never lead to any notion common to all living beings taken together. Anatomy indeed was given up to the study of the different parts which could be distinguished by the naked eye or with the magnifying glass in animals and plants ; and there was no need of being a great scholar to remark the want of likeness between the bones, muscles, and nerves of the dog on the one hand and the stamens, pistil, and leaves of the strawberry plant on the other. Comparative anatomy was necessarily limited to restricted groups such as vertebrates or mollusks.

The perfecting of instruments of study, particularly of the microscope, has made it possible for students to penetrate more deeply and intimately animal and vegetable structures. The first important conquest of biology, due to the perfecting 
of the microscope, was the cellular theory, which was thus expressed :

Every living being is composed of one or several cells.

This was a general law which concerned the totality of animals and plants : it was therefore a chapter of Biology. We shall see what is to be thought, in strict science, of this unity of structure expressed by the cellular theory; but, from the start, it gives us an important idea concerning the dimension of the structural phenomena that are possible characters of life. The first structural phenomena which are really common to all living beings, as discovered by means of the microscope in going down the scale of measurable magnitudes, belong to the order of cellular dimensions, that is, they are already far below a millimetre. Without insisting here on the exact dimension, the mere discovery that such a dimension exists at once gave the idea that phenomena really characteristic of life take place within the neighbourhood of a certain degree of the scale of magnitudes. Anatomy, thus transported by the aid of the microscope into this particular region of measurable things, now took the name of Histology or Cellular Anatomy. Only this part of anatomy can furnish Biology with appreciable results.

It was easier for physiology to give results of a general order; questions of nutrition and respiration soon showed the importance of Chemistry in the realization of vital phenomena. One of the biological conclusions earliest known was the necessity of oxygen for maintaining life both in animals and plants; therefore there is chemistry in life, that is, phenomena that take place on a scale of magnitudes below that of cellular dimension, in the order of dimension of molecules and atoms. This second conquest of science helped to assign the place of life more exactly among the other phenomena of nature; we see from it that biological 
phenomena properly so called, while they are not found above the dimension of the cell, do extend to smaller manifestations of atomic or chemical order. We shall have to seek the lower limit of dimension of biological phenomena; it may be given us in the dimension of the least radiations of ether which are able to influence vital phenomena. We must be content with this first approximation for the present, keeping hold however of this very important fact-before everything else in the study of biological phenomena comes the question of a scale of magnitudes.

Some of the great biological laws, that of the heredity of acquired characters for example, at first sight seem not to fall under this necessity of being connected with phenomena placed within restricted limits of dimension. But if such laws are really biological, that is, general, we may be sure beforehand that the means by which they manifest themselves will belong to the order of magnitude of biological phenomena; and so heredity, a resemblance among beings as large as you please, will be realized by means of the egg, which is a cell.

Embryology gives no new biological results for the reason that, having only recently become a science and being the child of the cellular theory, it is still distinguished with difficulty from histology. Embryology is the histology of very young beings. 
NATURAL PHENOMENA GROUP THEMSELVES IN PARALLEL SERIES OF DIFFERENT SCALES OF MAGNITUDE

A SLIGHT consideration of the dimension of biological phenomena has given us a glimpse of the importance of questions concerning scales of magnitude in the objective study of nature. The principle of continuity as we have defined it allows us to look upon organic evolution as a natural sequence of inorganic evolution; it also obliges us to ask ourselves at what particular point of inorganic evolution we can reasonably place the appearance of life. But to do this we must pass in rapid review inorganic evolution or, to use language without any hypothesis, the general classification of natural phenomena.

The principle of continuity is wonderfully verified in the manifestations of inorganic activity taken altogether; but this is on condition that we do not try to bring everything into a single series. On the contrary, we must content ourselves with verifying several parallel series with different scales.

It is a strange thing that nature, whom we like to think so rich, has at her disposition so small a number of models ; and these we find so like each other in the corresponding terms of the parallel series that the mathematical formulae applicable to one of the series can be used for the others without any great modification. Even the modern theory 
of electrons finds in an atom a very minute representation of the planetary system, with a positive central sun and negative little planets turning around it. A few examples will help to understand this existence of parallel series of different scales; I choose them, of course, among those which have some immediate relation with life, so that the study of them may be of immediate use.

First Example: Vibratory, Oscillatory, Periodic Movements.

Vibratory motion, which occupies so considerable a place in science nowadays, was first verified in the phenomena of sound. The first vibratory movement known was that of a spring producing a sound. In the study of acoustics, scholars found a first continuous series of phenomena all comparable with each other and differing from each other by simple numerical coefficients. Every one knows this series-the series of sounds classified in the order of height and reaching from the highest-pitched sound to the lowest perceptible to the human ear. The study of such vibratory movements is no longer carried on by the ear, classifying the height of sounds, but by the eye which verifies the registering of sound movements on cylinders ; and this allows us to complete the series at its two ends by vibratory motion which is quicker than that of the highest perceptible sound, or slower than that of the deepest perceptible sound.

Here, then, we have a series which is complete and very interesting in itself; but it became still more interesting when the genius of Fresnel, taking up an idea of Descartes and Huyghens, found in it a model for those movements of the ether of physicists which constitute what we now call radiations. Clerk Maxwell has since modified this idea of Fresnel and substituted for the conception of an oscil- 
latory movement of the ether that of a periodic oscillation of its electro-magnetic state. However this may be, the original formulae are still valid; the model of vibratory motion has not changed.

In these vibratory movements of ether there is found a series parallel to the series of sonorous vibrations although entirely distinct from it, and infinitely more important. This series has for its primitive nucleus all those luminous radiations which form the spectrum of white light. It has been increased by the discovery of the infra-red and ultraviolet rays, and again by that of the $\mathrm{X}$-rays in the order of very rapid vibrations, and particularly, in the order of very slow vibrations, by the discovery of the wonderful oseillations of Hertz (the Hertzian waves of wireless telegraphy), which are like a tangible verification of Maxwell's theory.

This series is not yet completely continuous; certain degrees in the scale are wanting to it, for example, at the infra-red and ultra-violet ends; but the wonderful continuity which exists within the extent of visible spectra is a pledge of future discoveries.

It would be possible in an order of much larger phenomena, to find the same model of periodic movements in the planetary revolutions; but this would scarcely interest us from the point of view of the study of life, which has nothing to do, at least directly, with the ork its of the stars.

On the contrary, sound vibrations and light vibrations, although they belong to two parallel series entirely distinct, are very useful to us in locating life in nature; for living substances may be directly sensitive to these two orders of vibrations. Animals see ${ }^{1}$ and hear ; therefore they receive impressions from phenomena belonging to two series so

1 In unicellular animals without eyes we sturly their sensitiveness to light under the name of " phototactism." 
very different in their dimensions. And this simple observation already shows that life is not localized in one point of a series of natural phenomena, but occupies a considerable place in the general activities of the world. When we see a rainbow forming in the spray of a fountain, we find that the movement of liquid drops does not displace the luminous apparition; the second phenomenon is therefore absolutely independent of the first, whose rapidity belongs to the same order of magnitude as sound movements. Life, which is sensitive to movements in both these two parallel series, offers us in like manner two sorts of distinct activities corresponding roughly with the rainbow light and the spray of the fountain.

Second Example: Chemistry, Colloids, Nebulae.

Our first example was taken from oscillatory movements ; the second concerns structures, mechanisms, which are comparable, at least provisionally, to buildings constructed by men and owing their particular properties to the manner in which they are constructed.

Now that the atomic theory has conquered the world, we can define chemistry as " the science of molecular edifices built up with atoms, and the study of the conditions in which such edifices destroy and construct each other." Chemical phenomena, accordingly, are of the order of magnitude of atoms, or, at least, of the distances which separate atoms in the molecules or molecules from each other.

For some years the attention of scholars has been particularly drawn to special bodies called colloids, which are neither frankly solid nor frankly liquid; their name comes to them from a comparison between such bodies and a watery solution of glue. Theory, at first, and microscopic observation in diffracted light afterwards, have shown that these colloids result from the existence of particles 
held in suspension in a fluid called a solvent; often they may be taken as minute spherules formed of a different fluid. Here, too, is a phenomenon of structure; but it is of dimensions quite different from those of the molecular or chemical phenomenon; each particle in suspension in a colloid contains a very great number of molecules.

Finally, a nebula in the heavens may be considered a giant colloid; but nebulae have no direct relation with life, whereas molecules and true colloids, on the contrary, very particularly concern the biologist.

One of the first conquests of biology properly so called was due to Dujardin, who asserted that all living beings are formed of sarcode; later, the word protoplasm was substituted for sarcode, and its use has prevailed in science. But it must be acknowledged that the unity of the word protoplasm at first veiled an error; it was believed that protoplasm was the same in all living bodies, and so we had only to say that all living bodies were of the same statethe protoplasmic state.

The expression, also, was very vague. A few years ago, if a biologist had been asked to define the protoplasmic state-an expression of which he made daily and copious use-he would have had to answer that a state is said to be protoplasmic where we find substances living; but that there are many differences, even in mere look, between the protoplasm of amoebae and that of bacteria. The word protoplasm was, therefore, a synonym of "the particular phys:cal state of living substances "; and Dujardin's discovery amounted to this, which was not without value:- there is such similarity of state among different living substances that, from a structural point of view, we may bring them under a single denomination.

Recent science allows us to define more exactly that 
which Dujardin discovered intuitively. Nowadays we say-protoplasms are colloids-which amounts to asserting that every substance, while living, is in the colloid state. The interest of this simple proposition is beyond measure; it is the keystone of all biology.

Let us not forget, however, that one of the first conquests of comparative physiology was to establish the existence of many chemical phenomena among vital phenomena. Now chemistry deals with atomic or molecular dimensions ; the colloid state, on the contrary, relates to activities of a dimension far superior to that of molecular reactions; and so we find, for the second time, this remarkable character in biological phenomena, that they take place, so to speak, at one and the same time, along two different scales of magnitude. In fact, as we have already remarked, living substances which are sensitive to sound are also sensitive to light; in like manner, we now see that vital reactions are at one and the same time of chemical and colloid order ; and so by an hypothesis, which may seem bold, but which opens vast horizons before us, we may suppose that, if light acts on the chemical reactions of life, sound acts on its colloidal manifestations.

A most important conclusion which already seems to result from this very recent study of colloids - a conclusion which will become more and more clear in the following pages-is that, in many cases, the chemical reactions produced between the particles in suspension and the liquid solvent help to modify the nature of the colloids considered as colloids; in other words, they have an influence on the colloidal state special to the particular colloid. Reciprocally, if direct actions modify the colloidal state, there may be a result in chemical variations, molecular reactions, between the suspended particles and the solvent. 
This influence of the colloidal state on the chemical state, and the reciprocal influence of the chemical state on the colloidal state, seem to be the rule in living colloids. The fact may be expressed more clearly in the copious terms of equilibrium-terms which, since the admirable studies of Willard Gibbs, promise to become some day the language of universal mechanics.

Let us consider three spherules A, B, C, in suspension in the solvent $\mathrm{D}$ of a colloid. We may consider the equilibrium of the system, either from the colloidal point of view, that is, from the point of view of the relations of position

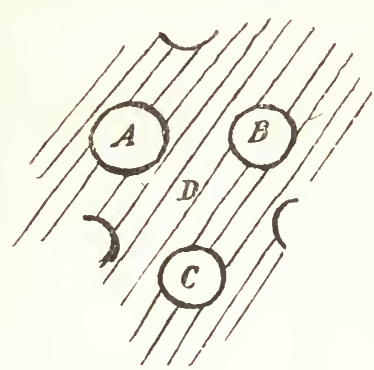

Fig. 1. of the spherules A, B, C with each other, or from the point of view of the osmotic and chemical exchanges which take place between each of the spherules and the solvent (Fig. 1).

If the chemical reactions take place between the particles and the solvent, the result may be this or that variation, of electrical order for example, bringing the particles nearer together, and so a modification of the chemical equilibrium will bring with it a corresponding modification of the colloidal equilibrium. Reciprocally, if some influence capable of modifying directly the colloidal equilibrium should force the particles A, B, C nearer together, this might result in a modification of the osmotic and chemical equilibrium existing between these particles and the solvent.

Any agent, acting directly on a colloid, may exert influence according to its own dimension, either on the colloidal equilibrium or on the chemical equilibrium existing between the particles and the solvent; but as these two orders of 
equilibrium are bound together by relations of cause and effect, it will happen in the long run that an external agent will be able to produce in a colloid a result of a very different dimension from its own. A sound vibration, for example, may determine secondarily a chemical modification from having set the suspended particles in motion; reciprocally, a luminous vibration may determine secondarily a modification of the colloidal state, from having directly produced certain chemical reactions. And yet sound vibrations are too great in dimension with respect to chemical phenomena, and light vibrations, on the contrary, are too small with respect to the particles of the colloids, to be able directly to produce that effect which they determine secondarily.

Thus not only does life, if I may use the expression, bestride two series of phenomena so widely separated as sound vibrations and luminous vibrations on the one hand, and on the other molecular and chemical reactions and particle or colloidal variations; but life also sets up a connexion between these two series of phenomena, which should seem utter strangers to each other. These are essential facts, and we shall have to come back to them. 


\section{CHAPTER IV}

\section{THE DIMENSION OF LIFE OR THE PLACE OF LIFE IN NATURE}

WE have now our tools for locating in part biological phenomena among the other phenomena of nature; we know that they have a place between the phenomena of equilibrium peculiar to colloids and the chemical phenomena of molecular equilibrium. Moreover, we shall be led to a more exact localization when we find what physical agents are capable of influencing living substances. For example, it interests us to know within what limits of vibratory velocity sound movements are capable of influencing protoplasms, and within what limits of wave-length certain vibrations analogous to light vibrations can modify the chemical substances which constitute protoplasm.

r. We do not yet know the essential biological phenomenon, but we can foresee its dimension.

There are chemical reactions outside of life; there are colloid bodies which are not living; therefore none of the characters so far considered will allow us to define life with relation to not-living substances. We only know that every living substance is in the colloid state and is the seat of chemical reactions, and that there is a relation of cause and effect between its colloidal equilibrium and its chemical activity-and this is a great deal.

There are measurable quantities besides those which come back to measures of length. Temperature, for example, 
is a quantity that we know how to estimate with great exactness. Now the biological phenomenon is very clearly localized in the scale of temperatures; we can assign a lower and a higher limit of temperature, limits outside of which no protoplasmic life can appear. It is very nearly between $0^{\circ}$ and $60^{\circ}$ Centigrade that all vital activities properly so called are manifested, which does not mean that living substances are inevitably killed outside of these limits ; on the contrary, at least under certain forms, certain living substances can resist very rigorous cold and temperatures higher than $100^{\circ}$ Centigrade (212 $2^{\circ}$ Fahrenheit). This already leads us to distinguish the property of "being alive " from the property "able to live"; the second is naturally more elastic than the first. But vital activity properly so called may be boldly placed within the limits of $60^{\circ}$ Centigrade and the neighbourhood of zero (freezing point).

Without some little reflection on the real value of these limits we might imagine that life occupies a large space in the scale of temperatures; we might even be astonished that life begins almost identically with positive temperature. The reason is that man, who is a living being, has chosen his scale of temperatures for his own use and in accordance with his own nature. He has taken as fixed points of his scale the extreme points between which, under the pressure familiar to him, water remains liquid ${ }^{1}$ and it is quite natural that life should be localized more or less between the same fixed points, since protoplasm is a watery colloid that needs liquid water for its existence. The whole history of life is bound up with that of liquid water ; life is an aquatic phenomenon.

1 Waters having salts in solution have not the same freezing temperature; this is why we have to give zero as being only about the lower limit which permits vital protoplasmic activities. 
Nowadays we can obtain in laboratories temperatures far lower and far higher than those known to our ancestors ; we can also recognize in certain stars the existence of temperatures higher even than that of the electric furnace. Hence life, limited to the temperature of liquid water, appears to occupy in the scale of temperatures as restricted an interval as it does in the scale of magnitudes.

In order that life should exist on the earth's surface, the latter must have limits of temperature very exactly fixed. This is why life has not always existed on the earth. It could appear there only when the surface temperature had become low enough for water to be liquid; it cannot endure when the temperature becomes too low. Life is only a surface accident in the history of the thermic evolution of the globe.

This question of temperature is essential in all that concerns phenomena which, like vital phenomena, comprise chemical activities. We know, indeed, that chemical reaction between two given compound bodies takes place only at a certain temperature; under different conditions it may not take place at all, or even turn out just the contrary. Now we have seen that a very important character of living colloids seems to consist in the fact that there is a relation of cause and effect between the colloidal equilibrium of these substances and the chemical equilibrium of their constituent parts. With these particular chemical constituents, therefore, the conditions of existence have to be such, at the temperature of life, that slight variations in the colloid state (electrostatic state, hydrostatic state, etc.) may intervene to set up chemical reactions among these constituents, or even to change the direction of reactions already existing. In other words, in the thermic conditions of protoplasmic life, at least a certain number of the chemical 
constituents of the protoplasm must exist at the temperature of dissociation.

The fundamental experiment of dissociation is well known. Above $960^{\circ}$ Centigrade carbonate of lime contained in a close vessel with carbonic acid and lime exists in equilibrium with these its two constituent substances, just as water in a close vessel is in equilibrium with its own steam. Increase slightly the pressure of the enclosing surface without change in temperature, and the carbonic acid will combine with the lime to form a new quantity of carbonate of lime : diminish the pressure, on the contrary, or, the pressure remaining constant, increase the temperature, and the carbonate of lime will dissociate itself into lime and carbonic acid. Every variation in temperature or pressure will thus be accompanied by a formation or a dissociation of carbonate of lime; the chemical equilibrium existing between the compound and its composing elements will therefore be governed by the physical conditions existing within the enclosed surface. Here we have chemistry and physics united by relations of cause and effect; and so inseparable are they that scholars have had the idea of coining a new term-chemical physics-to designate the science treating of these phenomena which stand astride on chemistry and physics.

We can sum up, therefore, what has been so far said, in a single formula-the study of life belongs to chemical physics. 


\section{CHAPTER V}

\section{REVERSIBILITY}

The fact of chemical dissociation above a certain temperature is often expressed by saying - corresponding phenomena are reversible.

Given two phenomena A and B (in our case, phenomenon $\mathrm{A}$ is the simultaneous presence of carbonate of lime, carbonic acid and lime within a closed space; phenomenon B is the existence in the same enclosure of a determined temperature and pressure), we can say that these two phenomena form a reversible whole under the following conditions :

From direct interference producing in phenomenon B a modification $b$ there will result in phenomenon A a modification $a$. Inversely, direct interference producing in phenomenon A a modification $a$ will result for phenomenon $\mathrm{B}$ in the same modification $b$ which had previously brought about in A the modification $a$. In other words, effects become causes and causes become effects.

The existence of this reversibility, which has been established with more or less completeness, between the colloidal state of protoplasms and the chemical nature of their constituent substances rules all biology.

Colloidal activity and chemical activity are exercised, as we have seen, at different degrees of a scale of magnitudes. Consequently, colloidal activity may be influenced by 
external phenomena of an order of magnitudes such as have no direct influence on chemical activities. But there is a repercussion of the colloidal variations on the chemical activities, and, vice versa, external phenomena which act directly on chemical phenomena may, secondarily, have a rebound in colloidal activity.

Now, the external phenomena belonging to an order of magnitudes able to act directly on colloidal activity comprise, as we shall see, what we properly call the acts of animals, that is, their movements-locomotion for example, which we can see directly with our eyes, with or without a magnifying glass according to the case. A first consequence is that the acts of animals, through the intermediary of the colloid mechanism of protoplasm, can influence the chemical equilibrium even of their constituent substances. In other words, the repercussion of these acts may be stored up in the intimate chemistry of living substances, just as the energy of a waterfall is stored up by the intermediary of a dynamo in the accumulator with its lead plates. The accumulator having been charged under the chemical form of lead peroxide is able under varying conditions to give back, at the expense of its provision of peroxide, a part of the energy of the waterfall.

Taking a great step forward and passing over the numerous intermediaries, we can draw from these general considerations an extremely interesting conclusion, though rather premature at present. If the hen fabricates the egg, the egg in its turn will fabricate the hen. We shall not therefore be astonished when we come to verify the marvellous phenomenon which governs the entire evolution of living beings : the heredity of acquired characters. 
Analysis of Natural Phenomena 


\section{CHAPTER VI}

\section{DANGERS OF TOO HASTY ANALYSIS}

IT might be thought that the previous chapters had prepared us to discover what we seek, namely-What are the esseritial biological phenomena, the characters, which distinguish living bodies from not-living bodies?

In point of fact, we are now aware that in biological phenomena there are chemical activities and colloidal activities. If we could only say what chemical activities and what colloidal activities, we should very likely be able to separate living bodies from not-living bodies.

Unfortunately, in the actual state of chemistry and physics, we cannot answer such questions. We cannot say by what chemical or colloid peculiarity the living being differs from its corpse. So far we can think of defining life only by its results. But we are certain that such a definition is possible, since in common life the most ignorant men do not hesitate in the greater number of cases to assert that one body is living and that another is not. There must therefore be objective characters, quite open to research, which answer the question.

We must analyse all that we know of the manner of being of living bodies, in order to find out that which belongs to them and to them alone; but we must choose our method of analysis so as to avoid errors which, by leading us into mistakes in language, would make all our efforts barren from the start. 
To show the dangers of too hasty analysis, the best example is found in two very celebrated theories which still encumber the field of biology and which, owing to errors of method, still weigh heavily on the student's shoulders. These two theories render quite valueless for purposes of research those two phenomena which best inform us of life. I mean, first, heredity, and, secondly, the fabrication of specific antitoxic serums.

The scholars responsible for such errors of method have, on the other hand and very justly, a very great scientific authority; and this has rendered their theories even more fatal. The first theory relates to heredity and is due to Darwin and Weismann: the second relates to antitoxic serums and is due to Ehrlich. In both cases the error of method is the same; it consists in representing by a name things which do not exist.

The problem of heredity, which we have to study later on, may be stated as follows: How is it that a herring's egg, developing amid the hazards of the sea, gives birth to a herring ? Evidently there is something in it which distinguishes it from the egg of a sea-urchin, since the two eggs developing under similar conditions give birth to such dissimilar animals. It is this unknown something which we may call the specific heredity of the herring's egg.

Darwin, when he wished to explain the mysterious nature of such heredity, most likely reasoned as follows : If I had to reproduce a herring myself, I should need a complete description of all its characters; in the same way the egg must possess, if not a description, at least a representation of all the characters which are necessary to constitute the adult herring that is to come out of it.

Such reasoning is not wanting in logic ; but we must come to an understanding about the word character. What we 
call the characters of a living being or not-living body are the elements of the analytical description which we make of them for our personal needs and according to the caprice of our fancy. Such characters, therefore, exist only inasmuch as we create them for our use. We can describe the herring in a hundred thousand different ways, by cutting it up in small cubes or in thin slices, the contents of which we analyse successively. Darwin chose the division into cells; and he consequently supposed that each cell of the herring's body is represented in the egg by an invisible gemmule which is its representative particle.

Although the division into cells has a more biological look than a division into thin slices or parallelopipedons, Darwin none the less chose it at random. Consequently it had few chances to be exactly that analysis of the herring which is represented in the egg; and, previous to the cellular theory, some other Darwin might have imagined a quite different representation. A lover of simplicity might even reduce the analysis of the herring to a greatly condensed expression by asserting that the herring has only one character, namely, herringhood, or the property of being a herring. Then it would be sufficient that this single character should have a determinate existence in the egg; ard this we are sure it has since it is the very expression of the phenomenon of heredity. Darwin for a single herringhood substituted several million cellhoods, quite as mysterious and much more hypothetical.

Every one agrees as to this fact-the egg contains the elements necessary to determine the existence of the adult under those conditions in which its development takes place. But this precisely is the one important thing to know -What are these necessary elements? In other words, by what process must we analyse the herring in order that its 
reproduction, due to an egg issuing from it, should be comprehensible to us? Darwin, and Weismann after him, advocate cellular analysis; but there are any number of other analyses having just as many philosophical reasons why they should be good-and chance will never make us find the best.

Weismann aggravates the error of Darwin's method in his desire to make it strictly exact. He imagines in the cells themselves characters represented by particles still more minute than the gemmules; and he does not see that to represent a character by a particle amounts to giving to the character, which is only a product of the analyst's fancy, an absolute existence. This giving of names to things which do not exist is beyond remedy ; for the names go on existing in language and people cannot believe they mean nothing. Weismann's language nowadays is used by the majority of histologists.

The mistake made by Ehrlich is of the same kind and quite as fatal in the realm of general pathology. When you inject into a living animal a colloid taken from another animal or from microbes (poison, toxin) the animal, if it remains alive, undergoes a particular modification. This is shown by the fact that its serum acquires the property of neutralizing henceforward the action of the toxin or poison.

Without asking whether in this conflict of colloid substances the phenomenon produced belongs to the colloidal or the chemical order, Ehrlich straightway accepted as a fact that, in the serum of the animal receiving the injection, there appears a chemically defined substance-and precisely that substance which is the antidote of the toxin, the latter being also considered a chemical agent.

This manner of presenting facts bestows quite gratuitously on the animal's cellular elements a veritable genius 
for chemistry, enabling them in the presence of thousands of chemical poisons always to fabricate the specific antidote. But Ehrlich's theory is also objectionable because it renders barren one of our most powerful means of biological investigation.

It is not mere chance that has led me to choose these two examples of typical errors of method. Heredity and the process by which specific antitoxic serums come into existence are to be for us the two fundamental phenomena of biology. 


\section{CHAPTER VII}

\section{ARTIFICIAL AND NATURAL METHODS}

When we have to study a fact taken by itself we are free to choose that method of analysing the fact which pleases us ; the best is that which is clearest for us as observers. From such a point of view all methods of analysis are artificial.

It is not the same when we have to study one object with reference to another object, with which it has relations. Then our method of analysis of the first object must be such as to bring out precisely those elements of that first object which are in relation with the second.

To go back to our example, if there had been question simply of describing an adult herring, we should have had no reason beforehand for chocsing one mode of analysis rather than another. We should naturally choose the most convenient among the several descriptive methods, all of which would be equally artificial.

But when there is question of a herring's heredity, that is, of the herring considered in reference to the egg which it produces and which will produce it, there must be a natural method; and this will consist in bringing out clearly in the herring just those among its characters which were determined in the egg-and no others. In such a problem the discovery of the natural method amounts to solving the problem itself.

There are some celebrated examples which will bring 
home to us this question of artificial and natural methods.

First Example: The Analysis of Audition by Helmholtz and by Pierre Bonnier.

The first example and the most suggestive of all occurs in the analysis of sounds. According as we study sounds in themselves, as vibratory movements, or in reference to the human ear which perceives them, we find ourselves in the first or in the second case already indicated, that is, with absolute freedom for artificial analysis or forced to a natural method of investigation.

For the analysis of very complicated sounds, such as the human voice, two methods have chiefly been in use :

1. The graphic method, which consists in registering on a turning cylinder a sinuous line traced by a stylus attached to a plate made to vibrate by the voice. This method has accredited itself, since the invention of the phonograph allows us to reproduce the human voice thus registered on a cylinder.

2. The method of resonators, which consists in decomposing a complex sound into simple sounds, just as by the prism white light is decomposed into simple lights. Helmholtz successfully applied this method to the study of the human voice. He arranged in a room a great number of resonators, each giving a determinate simple sound; these resonators were covered with a light layer of dust and a singer placed near them uttered the sound of the vowel A, for example. The figures traced in the dust on the resonators made known which of them had vibrated ; the corresponding simple sounds were noted and thus it was discovered which simple sounds by their superposition produce the vowel A. Morecver, it was easy to verify the validity of such an analysis by making all the resonators noted vibrate together-for this reproduced the vowel A. 
These two methods of analysis-the method by inscription on a cylinder and the method by Helmholtz's resonators -were therefore equally scientific so long as sound was only to be studied objectively as a vibratory movement. We need not say that the method of Helmholtz was more satisfying to the human mind.

Quite different is the problem of explaining the mechanism of audition, that is, the relations of sound with the human ear. There is no longer any question of knowing which kind of analysis is more satisfactory to the human mind, but which will adequately analyse the function peculiar to our auditive apparatus. Making a mistake which was like that of Darwin and Weismann, Helmholtz took it for granted that the analysis of sounds by our ear is made by the method most satisfying to our mind ; and so he assigned to the fibres of Corti in the internal ear the office of resonators, each of them being able to reproduce a simple sound. It has been demonstrated that this hypothesis cannot be accepted, yet it is so pleasing that it is still taught everywhere.

Pierre Bonnier, on the contrary, put forth the hypothesis that the analysis of sound by the ear is made in a way analogous to that of registering on a cylinder. $\mathrm{He}$ considered that the extremities of our auditive nerve receive the impression from the form of the vibration of the air-a form transmitted by a series of pressures to the intra-uricular liquids, just as it is transmitted to the vibrating plate from which the stylus transcribes it on the registering cylinder of the phonograph.

Pierre Bonnier's theory seems preferable to that of Helmholtz, but it is not the place here to discuss the relative value of their systems : I merely wish to show that there is no reason why that analytical process which is more pleas- 
ing to the human mind should be chosen by our ear in collecting sounds. For the sake of consistency in this question of methods, I will even say that, although Pierre Bonnier's theory seems acceptable to me, it is possible that the ear uses neither the method of resonators nor that of registering sonorous forms, but some third method not yet discovered which, though perfectly adapted to our auditive mechanism, would probably be of little use in teaching acoustics in school.

Second Example: Analysis of a Chemical Compound into its Elements or Functions.

In the study of a chemical compound the end proposed may be either to describe it so completely as to be able to recognize it afterwards wherever and whenever it is found, or to characterize its behaviour toward this or that other compound previously chosen.

In the first case, any and every means of analysis will surely be good if exact. You measure the density of the body you study, its boiling and freezing temperature; you estimate as closely as possible its colour, taste and smell; you make its chemical analysis into its elements and establish how much carbon it contains, how much sulphur, how much hydrogen, and so on. In a word, you gather together all the characters you need; each may be useful in a given case, especially in pharmacies where the only need is to be sure of the nature of a product. But if you only gather up in this chance way all the descriptive characters of the body you are studying, you will in no wise be fitted to foresee how the body will behave in presence of sulphuric acid, for example, for the analysis of the body from the sulphuric-acid point of view has probably nothing in common with its analysis from the human point of view. On the other hand, a very simple process will enable you to resolve 
this new problem; you set up a reaction between a certain quantity of the body you are studying and sulphuric acid -and you note what happens. This is what the old chemistry did; you will find in the books of twenty years ago a description of each body, first of its distinctive characters, and then of its reactions with a certain number of chemical agents chosen beforehand.

Advances in atomic chemistry enable us to condense in few words or in stereochemical formulae, whatever it is possible to foresee with regard to the reactions of one body with all other possible bodies. Now that this new kind of work has been accomplished, we say that the body has been analysed into its functions. The excellence of the method is this-such an analysis is not made from the point of view of the reactions of the body studied in connexion with a single body chosen beforehand, but with a view to all other chemical bodies whatever.

For example, in this new language, we say that glycerine is a triatomic alcohol having the primary alcohol function twice and the secondary alcohol function once. This enables a chemist to foresee its reactions with a host of other bodies ; but clearly it will be more convenient for a druggist to know that it is a syrupy liquid, colourless, with a certain taste and other qualities easily recognized.

Analysis into functions after this fashion is of priceless value to us in the study of life.

\section{Analysis of Bodies by Means of our Sense Organs}

Among the properties by which the chemists of other days recognized certain definite bodies, there stood apart as being easily used the organoleptic properties, that is, properties recognized by the direct use of our sense organs. Taste, colour, smell are in the front rank of these 
organoleptic qualities; it is easily understood that such qualities concern the relations of chemical bodies with man and not their relations with this or that definite compound. Accordingly such properties are useful only in man's direct knowledge of substances. We ought to remember that all our means of knowledge are, to a certain degree, organoleptic. Even when we measure rigorously by means of the most exact apparatus, when in other words we use science in its strictest methods, we are always decomposing the descriptions of things into elements which strike our senses. Length and thickness measured in centimetres, temperature measured by the thermometer and the rest are so many descriptive elements that concern our sight or sense of touch ; and, possibly, they may not be directly concerned in the relations between the body which we study and some other body than ourselves.

On this account we must distrust what is called the simplicity of things. A phenomenon which is simple to us may, on the contrary, be complicated in the extreme in relation to some other phenomenon with which it struggles in nature.

Reciprocally, and this especially is important to us here, a phenomenon very complicated in human analysis may have very simple relations with some other phenomenon of nature. For example, we shall see that, in a struggle between two different colloid states, phenomena appear which can be summed up in a simple formula, whereas the colloidal state itself appears to us men something so complicated that, in the present state of science, we are not yet able to give a direct definition of it.

So, when we study a new phenomenon, it is always best to abandon for the time being our human knowledge of the phenomenon and to seek for the other phenomena of nature 
with which it comes into relations capable of being expressed in a simple formula. In particular, we should understand the advantage there is in studying the struggle of phenomena with other phenomena of the same dimension as themselves -for example, of living bodies which are colloids with other colloid bodies living or not-living.

\section{The Method of Approximate Laws in Physics}

Though quite the opposite of that genuinely natural method of investigation which consists in the search after simple laws, the method of Approximate Laws is also of undoubted use in many cases. Its theoretic model is Helmholtz's Resonators, by which phenomena complex from the human point of view are decomposed into several other phenomena whose superposition reproduces the first, while each of them, taken separately, is susceptible of scientific analysis.

Suppose we draw a violin bow across a brass wire so stretched that it vibrates its whole length and produces what is called a simple sound. A similar wire, but this time of silver, would give the same sound in the same conditions. And yet the two sounds would differ by a special qualitytheir timbre.

To assert the identity of such sounds is to lay down an Approximate Law. The two sounds may be identical in pitch and in amplitude, and yet we distinguish one from the other because they have not the same timbre.

Helmholtz's method applied to the two cases enables us to discover that the fundamental sound in each is accompanied by different harmonics. But suppose we did not know how to use the resonators-we should be forced to assert that we had discovered an Approximate Law.

We do the same thing for a stone falling into a well. 
First we establish a simple law for the fall of a body in vacuo; but such a law can be only approximate the moment there is question of falling in air. Then we try to find another formula to express retardation from the friction of the air; and by this we correct our first formula.

In each case, for a real and single phenomenon which is complicated only in our analysis, we have substituted an artificial superposition of two imaginary phenomena, each of which it is easy for us to represent by formulae. This is the same fanciful method of analysis of which I spoke before ; it is useful only in the human study of a fact.

Mariotte's Law gives us an example of another Approximate Law; and in this case men of science have not yet been able to find a complementary formula to correct, for each gas, the inexactness of the law.

In spite of its imperfections, we shall make use of this method in studying life because it pleases the human mind. We shall even begin by it and devote the next part of our work to the application of this artificial method of Approximate Laws in seeking for the essential biological phenomenon. It will not upset readers who are accustomed to the method in their studies of physics and chemistry; and by means of it we shall discover the approximate law of assimilation or heredity.

In the next part we shall enter into the heart of our subject by applying the more rational method of studying the relation of living bodies with other colloids; and then we shall establish a law, this time rigorously scientific, which we may call functional assimilation, habit, or heredity of acquired characters. 


\section{PART III}

First Method of Analysis of Vital Phenomena

-The Approximate Law of Assimilation 


\section{CHAPTER VIII}

\section{ARTIFICIAL ANALYSIS AS IN THE PHYSICAL SCIENCES}

IN the present state of science we are able to define exactly neither the chemical structure nor the colloidal state peculiar to living substances-and yet this, doubtless, would be sufficient to characterize life. Hence we are obliged to look elsewhere. Naturally we think first of the cellular structure which was the first descriptive character that seemed common to all living beings. Indeed, such cellular structure is of considerable importance.

A character that seems quite as general as cellular structure is the presence in the living substance of each cell of two distinct colloid masses - one central, called the nucleus ; the other peripheral, called cytoplasm. The general existence of these two distinct masses, behaving differently in presence of one of the reagents used by us (basic aniline colours), makes us think of a mechanism in which the coexistence of two distinct agents, like copper and zinc in the Voltaic pile, would be necessary to realize the phenomenon of life.

Moreover, experiments which we shall have to describe later (merotomy) confirm our idea that the phenomenon of life does not take place when one of these agents is removed. So it is evident that we have here something fundamental ; the diagram (Fig. 2) is applicable to all living elements or to nearly all.

Unfortunately, here too we are stopped by a penury of 
means of investigation with regard to chemical structure and the colloid state. If we kill a cell by means of osmic acid or mercury bichloride, the existence of the two distinct colloids-the cytoplasm and the nucleus-becomes still plainer. In other words, we find the same structural character in the dead cell as in the living cell. From this we must not conclude that this structural character is not characteristic of life, but rather that, with our imperfect means of research, we cannot distinguish the living whole "cyto-

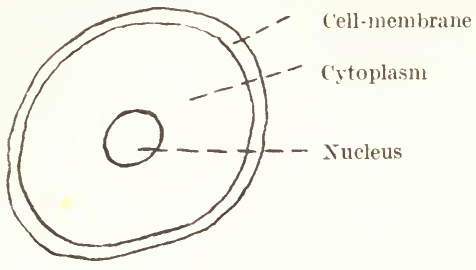

Fig. 2. plasm-nucleus" from the dead aggregate "corpse of the cytoplasm and corpse of the nucleus."

Let us seek elsewhere.

It was long believed that spontaneous movement was characteristic of life. It

has been found necessary to give up this opinion, first, because the pretended spontaneousness has been shown to be a result of chemical reactions and osmotic changes, and, then, because in dead substances such spontaneous movements have been discovered (Brownian Movement).

This question of the movement of living bodies has to be studied later; it is enough for the present to know that from its study we can derive the elements of the definition of life.

The living being grows, develops, dies, say the most ancient definitions. In this first character-growth-we find that which discriminates life; and this is all the more singular that the character is not observable in those beings which for us are, as it were, the very type of life, namely, adult men and animals.

In return, this character of growth is evident in familiar 
plants and also in animals, provided that we study the latter not at the very special moment when they are adults, but during the first time of their individual existence-precisely during what is called their period of growth. The calf grows and becomes a bull, the kid grows and becomes a goat, the child grows and becomes a man.

But you will say that the snowball grows by rolling, the alum crystal grows in a solution of alum, and yet we do not consider the alum crystal and the snowball living !

The case is not the same, for the alum crystal grows at the expense of a solution of alum, the snowball grows at the expense of a layer of snow, whereas the calf grows by eating hay, clover or lucern. In other words, the animal or plant develops at the expense of substances different from its own, whereas in the cases of growth observed in not-living bodies the increase in size is at the expense of substances identical with that which grows (or, at least, by means of elements which are always the same; for example, in the case of carbonate of lime which, at the temperature of dissociation, may increase under the influence of increased pressure at the expense of carbonic acid and lime).

Here, then, is a character which seems common to all living bodies, at least during a portion of their life.

It must be acknowledged that this character presents itself to us in very crude outlines; man is a grown-up child, but how different from the child! The bull is a grown-up calf, but not only his form, his substance even, his flesh, seem to us very different from the form and flesh of the calf. So we perceive how approximate only is this result : the calf from grass and lucern and clover produces the substance of the calf.

Yet beneath this crude proposition lies concealed the character by which we shall define life. It will be easier 
for us if we consider cases in which the identity of the original substance and of the final substance appears with greater evidence.

I sow a grain of wheat; I get from it a wheat ear with forty grains; then I sow these forty grains and get sixteen hundred ; thus, by two years' cultivation, I realize a production from the original one grain of wheat, at the expense of soil and atmosphere, of sixteen hundred grains whose substance seems to me very close to that of the original grain.

A more perfect example may be found in bacteria-culture as it is practised in laboratories. When a given bacterium has been well studied, when we know all its needs in respect to food, air, temperature, we succeed after minute care in obtaining a prodigious multiplication of the bacterium ; and we can preserve in its descendants, with the utmost exactness and detail, all the characters which had been brought out in the laboratory experiments.

In such a case the law appears without any vagueness of outline. The bacterium has assimilated its culture-medium, has transformed parts of this culture-medium into bacteria like to itself. If, then, we pass over the consideration of the form of the bacteria, if we attend solely to the substances which constitute the bacteria, we find a result of chemical order-a chemical reaction novel in its results and belonging to no not-living body.

Not-living bodies, not-living chemical compounds, are always destroyed by reacting chemically with other compounds. If we agree to take account only of the quantities of substance which have effectively undergone reaction, an equation of chemistry will always present itself, roughly, as follows :

$$
\begin{aligned}
& \mathrm{A}+\mathrm{B}=\mathrm{C}+\mathrm{D} \\
& 2 \mathrm{NaCl}+\mathrm{H}_{2} \mathrm{So}_{4}=\mathrm{Na}_{2} \mathrm{So}_{4}+2 \mathrm{HCl}
\end{aligned}
$$


That is, in the second member of this chemical equation you find none of the chemically defined bodies which appeared in the first member. $\mathrm{C}$ and $\mathrm{D}$ are always different from $\mathrm{A}$ and $\mathrm{B}$.

On the contrary, in the example of bacteria, the substance of the original bacterium which effectively reacted, since it gave the specific character to the reaction, is found, and considerably increased at that, in the second member of the chemical equation which represents the phenomenon.

To write symbolically this equation of a bacterium's multiplication, we must observe that such multiplication is made at the expense of certain nutritive substances which have disappeared from the culture-medium during the reaction; these substances I represent by the letter $Q$. At the same time that the bacteria multiply, there also appear in the medium new substances which we may call substances accessory to the assimilation, and which, always the same, are produced whenever the same bacterial species multiplies in the same conditions. I represent by the letter $\mathrm{R}$ these accessory substances of assimilation.

Now let $a$ be the sum total of the active substances of the bacteria, and suppose the reaction to stop when a number of bacteria has been formed (this number $\lambda$ being so much the greater as the reaction has continued longer, possibly equal to two, to four, eight, sixteen and soon, but always greater than one): the chemical equation representing the production of these new bacteria will be in accordance with the law of the conservation of matter:

$$
a+\mathrm{Q}=\lambda a+\mathrm{R}
$$

I call this the chemical equation of elementary life as manifested. I wrote out this equation for the first time ten 
years ago ${ }^{1}$ and it seems to me a handy expression for the law of assimilation.

Such a law of assimilation is rigorously exact in the very special case of a bacterium cultivated with all the precautions that prevent it from varying, but it is only approximate in other cases. Still, as Helmholtz has done for the timbre of sounds (see page 40), we can try to keep it scientifically exact by discovering what phenomena are superadded to the phenomenon represented by our equation; for these prevent it from corresponding with precision to what passes in living nature.

\section{Destruction and Variation}

Our success is assured if we observe that, even in inorganic chemistry, a definite compound is not limited to one way of reacting in presence of a certain number of other compounds equally definite ; the reactions which are produced in a mixture of bodies depend on the conditions realized. There is no reason beforehand why living substances should, from this point of view, differ from ordinary not-living substances. In our example of a bacterium which multiples without undergoing variation, we take precisely all the precautions necessary to avoid the production of a single reaction other than that which we wish to bring about. We realize experimental conditions analogous to those of a body falling in vacuo, and we obtain a formula as rigorously scientific as the mechanical formula of the fall of bodies.

But, on the other hand, we know that by treating a bacterium by other bodies than its culture-medium-by sal-ammoniac or mercury bichloride, which are also chemical substances-we no longer obtain a multiplication, but a

1 Théorie nouvelle de la Vie. Paris, Alcan, 3rd edition. 
destruction of the bacterium we are studying. This means that, in certain conditions properly chosen by a skilful experimenter, the bacterium by assimilation shows its quality as a living being; in other conditions, on the contrary, it behaves like ordinary chemical bodies and is destroyed while reacting. This destruction which the bacterium undergoes may, moreover, be brought about in any number of ways, according to the reagents used and the conditions in which they are used.

Finally, it must be further observed that destructive reactions are necessarily much easier to bring about than the constructive reaction; if you are given haphazard a bacterium and a liquid, and if you plunge the bacterium into the liquid, you are very much more likely to see it die than to see it live and prosper. Assimilation is the exception, destruction is the rule. A living body given up to change is much more likely to die than to live. No wonder that when we place it in the natural conditions where it is able to live, that is, to grow and multiply, it finds along with favourable conditions other harmful circumstances, save in the altogether exceptional case where I suppose the bacterium multiplying without variation.

Here we find with no great difficulty the cause of the mistake which generally makes the law of assimilation a merely approximate law. Destructive reactions peculiar to the living body under consideration, but not characteristic of it as a living being, are found in nature superposed with its assimilative reactions.

When the assimilative reactions are stronger than the destructive, the living being grows. When they balance each other exactly, the living being does not change perceptibly in dimensions, and we say that it is adult. But when, finally, the destructive reactions carry the day against assimilative 
reactions, the living being decreases and finishes by disappearing.

We are accustomed to say that a being is alive as long as assimilative reactions, no matter how slight, go on in it. Now what we have been saying proves at once that observation will often be unable to establish directly any visible growth. Growth can be verified only during the individual's period of growth; it disappears completely in the adult state and its place is taken by diminution during the period of decay which leads to death.

Thus, during the period of growth, assimilation is an approximate law tempered in its scientific rigour by destructive reactions ; in the adult state the corrections to be made in the law are as important as the law itself; and they become more important all through the period of decrepitude which leads to death. To try to find in this last period an example of the law of assimilation would be the same thing as to apply Marriotte's Law to a saturated vapour.

In certain cases it has been possible to separate experimentally the phenomena of assimilation from those phenomena of destruction which are regularly superposed in nature. This was done, for example, in the case of the bacteridia of anthrax; it is a fact to be remembered, as it helps us to an important idea.

1. At $35^{\circ} \mathrm{C}$, in a proper culture-medium, the bacteridia of anthrax multiply without any modification. This is an example of the rigorous application of the law of assimilation.

2. If you add to the culture-medium a small quantity of phenic acid or permanganate of potash, the bacteridia will still multiply but more slowly, and they undergo certain changes; a part of these we are able to study under the name of attenuation of virulence. This is an example of 
the approximate law - a phenomenon of destruction superposed with a phenomenon of assimilation.

3. The next step is even more interesting. In pure water, to which the same quantity of phenic acid is added as to the culture-medium in the preceding experiment, you sow the anthrax bacteridia-and the assimilative reaction is suppressed. Nothing remains but destruction, and the destruction is entire at the end of a certain time. But if you stop the destruction before the death of the bacteridia, you verify that the partial destruction of the bacteridia has produced the same attenuation of virulence as in Experiment No. 2. We can say, therefore, that, in Experiments 1 and 3, we have artificially separated the phenomena of assimilation and of destruction, the superposition of which gave us the result obtained in Experiment No. 2. This is Helmholtz's method of decomposing a complex sound into several simple sounds.

In this particular case, by exact experiment, we have caught in the act assimilative and destructive reactions superposing themselves. Hence we can assert with more confidence our approximate law of assimilation in cases where we are not able experimentally to separate the destructive reactions which accompany constructive phenomena.

This experiment of the attenuation of the virulence of anthrax bacteridia teaches us yet another thing.

The presence of phenic acid in the culture-medium not only slackens the multiplication of the bacteridia-something easily understood, because phenomena of destruction were added to the phenomena of assimilation. But, more than this, the bacteridia which we obtain have properties different from the original ones-they have varied. And, more still, if you next transfer them to a fresh bouillon, they will multiply with their acquired variation; and 
they will preserve it henceforward, until some other new variation is produced.

This wonderful fact of the conservation of acquired variations presents the greatest interest and will have to be studied later. Quite apart from it, we must here and now verify that, in living beings as simple as bacteria, partial destruction brings variation along with it. Such verification obliges us to acknowledge that the bacterium is not formed of a single and homogeneous substance, but of several elements susceptible of separate destructive attack. Otherwise, there would indeed be a diminution of the quality of bacteridia produced in the phenicated culture, but there would be no variation. A body's change in qualities under the influence of partial destruction of its substance proves that the qualities of the body are not inherent in a single substance, but in an aggregate of elements susceptible of separate destruction. An immediate consequence of this seems to be that variation in the qualities of a living being must result from variation in the respective qualities of its constituent elements. Our second method of investigation will enable us, in the fourth part of our book, to sift this idea more completely. 


\section{CHAPTER IX}

\section{USEFUL RESULT OF SUCH ANALYSIS IN GIVING LIFE ITS PLACE AMONG OTHER NATURAL PHENOMENA}

THE artificial analysis, which we have just tried by the method of approximate laws, is manifestly powerless with regard to certain problems which we shall soon resolve by a more rational method. But, at least, it has the advantage of bringing unity into language and enabling us to speak of biological facts in terms of chemistry. We have limited ourselves to verifying results without trying to find out how the results are got at. We could not do more in the course of our analysis, because we have not yet once occupied ourselves with the colloid state and morphological structure of living beings. We have only considered quantities in living substance, and we have only verified a result that concerns such quantities; that is, we have kept aloof from the particulars of the facts.

The same remark has to be made in every order of science ; the more general the language, the less adapted it is to the complete analysis of a specialized group of phenomena.

To our vital phenomena we have applied language adapted to the description of all chemical phenomena; and the fact that such language is sufficient to characterize life goes to prove that the particular essence of life is of chemical order. It gives life its place in chemistry; and this is already an important result. But it is also evident that 
such language, by the very fact that it applies to a thousand not-living phenomena, is not the language best adapted to the description of vital phenomena. In the case of alcohols we can also describe everything in the general language of chemistry; but if we wish a concise study of alcohols taken by themselves, we use the language of the function alcohol, which applies to alcohols alone. In the same way, the fourth part of this book will give us a language of functions - a truly biological language-which will enable us to reduce to a few words the general expression of all vital phenomena.

The approximate law of assimilation, by setting life in its place among other natural phenomena, satisfies us at first; but we have to acknowledge in many cases that its definition is very platonic. It enables us to recognize life in beings by means of long-continued observation, but even then only during the period of growth. In the adult state or in the period of decrepitude the phenomenon of assimilation, though we cannot doubt that it is still very real, is hidden by antagonistic phenomena.

If I see a cat, motionless and eyes ablaze, watching a sparrow, I know very well that it is alive; I recognize it by certain signs which do not deceive. And yet I have no means of assuring myself that phenomena of assimilation are going on in the cat's interior. The approximate law of assimilation helps me to know only the results of such phenomena taken as a whole; but they are accompanied by manifestations, at sight of which I recognize life without being able to define them exactly. It is also true that I may be deceived in my observation; a very clever taxidermist may have given the illusion of life by properly arranging the dead bodies of a cat and a sparrow. Possibly science may some day bestow on us an apparatus enabling 
us to distinguish by simple inspection the physical characteristics of substances which are alive. But, until such a bioscope becomes a reality, we shall have to be content with recognizing life by means certain only when observation has been long continued. 


\section{CHAPTER $\mathrm{X}$}

\section{DEFINITION OF ELEMENTARY LIFE IN CHEMICAL LANGUAGE}

IMPERFECT as may be the results obtained by our artificial method of analysis, they already enable us to give a partial definition of life. Clearly, this partial definition will comprise nothing that relates to colloidal or morphological phenomena, since it is made quite apart from any consideration of the form or structure of living bodies. Yet the definition will contain that which is essential, since outside of living beings we shall never find a phenomenon to which it is altogether applicable. It therefore fulfils one of the conditions by which we know a good definition.

We can give the name of elementary life to the special property of living beings which brings out clearly the specific reaction-assimilation-under proper conditions. A body is endowed with elementary life when at least some of its constituent substances, by reacting effectively in determinate conditions, are capable of quantitative augmentation while keeping their identity. And this, in other words, is when they are capable of assimilating foreign elements, taking the word " assimilate" in its etymological meaningto make like to oneself.

This partial definition refers to elementary life-a chemical property which, like all chemical properties, may or may not manifest itself according to the case.

With many living species we do not know the living 
substance in chemical repose; the living substance manifests itself to us only in uninterrupted activity, an activity that is assimilative or destructive according to the conditions. But with many other species, on the contrary, we can get the living substance in the state of chemical repose just as we get in chemists' bottles sulphate of soda or antipyrine.

These little morsels of living substance in chemical repose go by the name of spores. The spores of bacteria or of mushrooms are capable of remaining very long in chemical repose in a dry spot without loss of their elementary life, or at least losing it by very slow destructive reactions. But does not the same thing often happen to ordinary chemical substances preserved in a chemist's bottle? Duclaux was able to bring about germination in spores contained in air-dust filtered by Pasteur on dry cotton thirty years before, when he was experimenting with spontaneous generation.

With the higher animals we do not generally come across the living substance in chemical repose ; we find the tissues always under way of assimilation or destruction. Yet rotifers, little worms fairly advanced in organization, can be dried and, like spores, will keep for a long time the faculty of coming to life when furnished with water.

At the beginning of this chapter it was remarked that the definition of elementary life, as a chemical property deduced from the verification of quantitative chemical results, could have no direct reference to structural manifestations, of which no account had been taken in drawing up the definition. We might guess from the start that, elementary life being common to all living beings, it is likely that the existence of such a chemical property is related to the structural manifestations which are also 
common to all living bodies. But it does not follow from the definition. It is a theorem to be demonstrated by experiment - the morpho-biological theorem. Such a theorem we shall develop later.

On the other hand, the fact neglected in seeking for a chemical definition of elementary life-the phenomena of colloidal structure-makes us think that the approximate law of assimilation, which is so far purely chemical, may turn out more approximate than it seems when we take into account differences of colloidal state capable of masking it. When we spoke of the substance of calf or goat, we used expressions clearly inexact; for in the calf as in the goat there are liver and lights, muscle and nerve. But suppose for a moment what I hope the future will clearly show usthat liver and lights, muscle and nerve, are only different colloid states of identical chemical substances (the fact that the same tissues exist in all mammals is sufficient to make us consider the hypothesis plausible). Then the law of assimilation becomes almost as evident in the development of a child as in the multiplication of a bacterium. 


\section{CHAPTER XI}

\section{VARIOUS CONDITIONS IN WHICH A LIVING BODY MAY EXIST FROM THE CHEMICAL POINT OF VIEW}

We have accepted a definition of elementary life; and now we shall have to establish that, theoretically at least, the living substances of a living being may be found existing in three states, under three different conditions :

Condition No. 1.- The sum total of circumstances in which the elementary life of living substances manifests itself by strict assimilation without any destructive reaction or variation. We can rarely realize this condition perfectly, but we succeed in a few particular cases.

Condition No. 2.-One of the very numerous cases in which the living substance is the seat of destructive reaction. When these destructive reactions are produced alone, or if they win the day over the assimilative reactions, the fatal result is complete destruction, the death of the individual studied. In these reactions, moreover, living substances do not behave like living substances, but like dead or not-living substances which are always destroyed in reacting. The phenomena of Condition No. 2 are, therefore, phenomena of death.

Condition No. 3.-More or less perfect chemical repose, in which, when there is any reaction at all, it is never assimilative, but always a more or less slow destructive reaction accompanied by variations equally slow.

In reality, as we have seen, Condition No. 1 is very 
seldom fully realized in nature. For the most part it is accompanied by circumstances that superpose destructive reactions on the assimilative reactions and thus cause variations.

Strictly speaking, therefore, we do not often meet in nature with a phenomenon of elementary life manifesting itself, at least, not in its purity. In a being that lives there are at the same time phenomena of life and phenomena of death ; and the being remains alive only so long as the phenomena of death do not carry the day over the phenomena of life. In other words, the phenomena which take place in a living being can but rarely be translated into the exact formula :

$$
a+\mathrm{Q}=\lambda a+\mathrm{R} \text {. }
$$

For an exact account of what takes place we have to combine with this first formula of elementary life as it manifests itself one or more formulae of reactions destructive of the form:

$$
a+\mathrm{B}=\mathrm{C}+\mathrm{D} \text {. }
$$

Of course, this is factitious decomposition; but it is convenient in analysing the complex facts of life. We shall see later that, with a higher class of beings in whose life we observe periods of work and repose, there is not always superposition, but the alternation of Conditions No. 1 and No. 2. And, although it is contrary to a very widespread opinion, Condition No. 1 corresponds with the periods of work, whereas the greater number of physiologists admit with Claude Bernard the uear of living substances in their work-functional destruction. This error will be made clear in the next part of our book by using the rational method of decomposition into functions; it results chiefly from the existence, in the midst of living substances that are really active in the biological 


\section{METHODS}

phenomenon, of other not-living substances, called reserve substances.

\section{Reserves}

In the equation of elementary life as manifested we specified carefully that by the term $a$ we represent solely the really active substances which effectively intervene in the reaction we are studying. In fact, it is not enough for a substance to increase by the fact of a being's life in order that it should itself be living. For example, the substances of the term $\mathrm{R}$ in our equation-substances said to be accessory to assimilation or excrementitial substances-increase in proportion with the vital reactions, in which they have no part and which their accumulation may even prevent. This actually takes place with alcohol, which is an excrementitial substance of beer-yeast.

But it is not only the products of Condition No. 1 which are capable of increasing under the influence of life. Suppose one and the same Condition No. 2 to be constantly realized for a certain time without importance enough to arrest altogether the development of the living being we are studying; the products of this particular Condition No. 2, if not soluble and diffusible, will accumulate in the midst of the protoplasm. They are reserve substances, starch, fats, etc.

These substances, which are not living, are so incorporated with the protoplasm that their wear has given the idea it was the protoplasm which was wearing away. Now these reserve substances may commonly be used as $\mathrm{Q}$ substances, that is, food substances in elementary life as it is manifested in living elements.

A muscle which, by working, consumes its fat, grows thin, but it develops in so far as it is muscular substance. 
'The embryo contained in a grain of wheat consumes while germinating the starch reserve which was the result of a destruction of the living tissues of albumen, and uses it in fabricating the living protoplasm of the young wheat plant. Thus the destructive reactions of Condition No. 2, while they retard protoplasmic development, are not therefore lost for the individual; their result serves directly as food in future assimilations.

The action of chlorophyl, which is often mistakenly called assimilation, is a phenomenon of Condition No. 2 produced in green plants under light. A potato grows much faster in a cellar than in sunlight; but in the sunlight its tissues are charged with reserves which it will utilize later. 


\section{PART IV}

Second Method of Analysis : Decomposition into Functions-Strict Law of Functional Assimilation. 



\section{CHAPTER XII}

\section{ARTIFICIAL FUNCTIONS AND FUNCTIONS RATIONALLY DEFINED}

WHEN we wish a complete anatomical description of the body of a living being, we can separate it into parts in any number of ways. Just so, there is no reason why we should limit the number of methods by which we analyse the vital activity of the same being.

A living being is not like an industrial machine manufactured with the design of accomplishing a certain kind of work and able to do no other. A locomotive can exercise only the locomotive's function. On the contrary, a dog, a duck, a serpent, are able to manifest in a thousand different ways according to circumstances their specific activity as dog or duck or serpent. Now, circumstances so vary around any given animal and the animal itself changes so quickly that we may say without exaggeration an animal never does twice the same thing in the whole course of its existence.

Physiologists, however, when comparing the animal to an industrial machine, are accustomed to describe its activity by a decomposition into a certain number of functions which, from the descriptive point of view, are simpler than the total activity of the individual. Just so, in a locomotive we deduce from the backward and forward movements of the piston under the influence of steam pressure the transformation of the movement to and fro into a movement of rotation by the play of connecting rods and 
cranks and so on; and it would seem difficult for two engineers, each having to describe the locomotive's working, to decompose it in two different ways. Besides, the locomotive is an instrument conceived by man and executed by him with a view to a certain function.

On the contrary, when we have to decompose the total activity of a living being into partial and simpler functions, we should think there might be very many different ways of doing it-and so there are. But plyysiologists for the most part agree in using the same method and have thus created a language of analysis adopted by all.

To tell the truth, in an animal like man we find certain elements of action always comparable among themselves in the execution of a great number of different acts; they are like so many wheels in an industrial machine. Such, for example, are rigid bone segments, articulations, muscles, tendons, veins and arteries, nerves and the rest. Whenever a man's body executes a movement, we can decompose the movement into several parts corresponding to each of these elements of action. In such a case anatomy guides the physiological description of the phenomenon observed.

For the analysis of a locomotor act such decomposition is not only useful, it is indispensable. Yet it has many drawbacks. It leads the analyst to consider the different segments of the apparatus he is studying as independent of each other except in their relations with the mechanism, just as connecting-rods and cranks and wheels in the locomotive, each of which exists by itself, are independent of each other except in their relations with the mechanism. Now bones, muscles, nerves, are the living elements of one and the same organism and share in conditions of existence common to all. When a muscle contracts, it draws together two bone segments fixed to its two ex- 
tremities; but its contraction at the same time, and quite apart from this phenomenon which is obvious to the eye, acts on all conditions of equilibrium taken together $\overline{a s}$ realized in the whole individual. In other words, in a living being like man, there is not a single phenomenon, however local in appearance, which has not a general rebound in the whole organism. If I close my fist without executing a single other movement, I have no right to think that nothing takes place in the rest of my body relatively with this local movement. Consequently, if I decompose some obvious activity of the whole into a certain number of obvious local activities, I can be sure that my analysis is incomplete. I have neglected the rebound of each partial activity in the organism; and I can be sure that the sum of all the partial activities I am considering does not strictly represent the total phenomenon which I wish to analyse. So my analysis is bad, whereas with a locomotive a similar analysis would be satisfactory.

Nevertheless, this descriptive analysis of human activities is indispensable on account of the immense complexity of such activities; but we ought to be careful not to give it any absolute value. It is impossible to describe completely the flexion of the forearm without taking into account the state of the whole organism in which the flexion has its rebound, while the whole organism, in turn, has its rebound in the conditions of the local phenomenon.

It is the custom in treatises of physiology to study separately digestion, respiration, circulation, secretion and all the rest, 'although it is very certain that not one of these functions is exercised without the help of the others and without some influence over them. Such a method of analysis is, therefore, factitious, yet it renders great services for its own special need. In the preceding part of our 
book we have seen the conclusions which might be drawn as an approximate law from the application of this artificial method. But we have now to seek whether a natural method of decomposition into functions may not give us a more rigorously exact result from the biological point of view.

This idea of reaching a genuinely biological result-something truly general and applying to all living beings without exception-is to guide us in our search. Indeed, when we describe a phenomenon, if we bring in the flexion of the forearm, we shall certainly find nothing equivalent to it in an earth-worm or a sea-urchin having no articulated forearms. Physiologists aim precisely at this when they examine the great functions-respiration, circulation, digestion, etc.-found in all living beings without exception.

We have seen that such a method is artificial. That it is also susceptible of generalization merely proves that we can apply one and the same artificial method to the physiological study of all living beings. We ought not to separate from each other the different phenomena which take place at the same moment in a given animal. We must therefore resign ourselves to studying them all at once-something which demands a special synthetic language.

A dog, a duck, a serpent, as I have said, are capable of manifesting in a thousand different ways, according to circumstances, their specific activity as dog or duck or serpent. Yet their activity remains specific, that is, it goes on according to the peculiar structure of their organism and, under the same circumstances, a dog acts like a dog and a duck acts like a duck. It would be convenient, at least as a beginning, to create verbs corresponding with these different specific activities. For example, we would say a $\operatorname{dog} d o g s$, a duck ducks and so on ; and the question would be to know 
what are the different ways of dogging or ducking with relation to this or that circumstance.

For such odd verbs we may substitute the verb "to function," on condition that it be understood the word comprises all the specific activity of the being considered, under the circumstances considered. This manner of speech will give to the word function a meaning quite different from that of the physiologist. There will no longer be question of an artificial decomposition of the total activity of an individual into several simultaneous parts which we have no right to separate from each other. But it will be the decomposition of a succession of total activities, each of which is the result of two factors, the state of the animal we consider and the surrounding conditions taken all together at the moment we consider it.

Each function thus defined will be singular, differing from all others in the world. There are, indeed, too many elements in each animal and too many also in the circumstances which determine its acts, for a being of one species ever to find itself twice identically the same in identical circumstances. So it would be an illusion to look for something common in the sum total of functions $d o g$ defined in this way; they will vary ad infinitum. It would be even more impossible to find anything common to functions $d o g$, functions lizard, and functions pear-tree. Not even in the nature of these functions can we find a general law; but we may discover one in the linking together of the successive functions of one and the same individual and in the consequences to a given individual of its exercising a given function.

We have not at our disposition the imaginary bioscope which might enable us to recognize from mere inspection the physical state peculiar to living substances. Here too we 
must come back to observation of phenomena during a certain time. We do not study how the being lives-that is beyond our present means of investigation-but we do seek how the living being continues living. And in this search we hope to find a general law characterizing life.

\section{Organ and Function}

If our definition of function is accepted there will result from it a corresponding definition of orgun. The word organ has been abused in current language; and certain authors have not feared to express by the word any anatomically described part in the animal body, to say, for example, that the hand is an organ. In spite of all this, it is clear that the definition of organ must be physiological and the only possible definition is the following: an organ is the sum of the parts of an individual working together in the execution of a function. Those who believe in the existence of partial functions, of local phenomena having no rebound on the whole of the individual, may also believe in the existence of partial organs comprising only a part of the tissues of the animal. But if we go to the bottom of things, we verify the correlation uniting among themselves every instant all the regions of the living body. We are forced to say on the one hand-any organ whatsoever comprises the whole organism; and on the other hand-the rôle of this and that part of the individual has most importance in the constitution of a given organ.

Moreover, if our definition of functions considered as the total successive activities of one and the same living being is accepted, we ought to define an organ as the successive states of the organism corresponding to each function. And so life is a succession of functions ; the living being is a succession of organs. 
Let us designate $A_{1}, A_{2}, A_{3} \ldots$ the successive states of an individual, its successive organs, according to our definition; and $\mathrm{B}_{1}, \mathrm{~B}_{2}, \mathrm{~B}_{3}$... the successive sums total of surrounding circumstances which intervene in determining the activities of the individual. We shall have to consider any activity of our individual, any function whatever, as the result of two factors, its state A at the moment considered, and the sum total $\mathrm{B}$ of the corresponding circumstances.

In other words, any moment whatever of an individual's life may be represented by the symbolic formula:

$$
\mathrm{A} \times \mathrm{B}
$$

Life, all told, will be a succession of functions ${ }^{1}$, each of which corresponds with a formula :

$$
\begin{aligned}
& A_{1} \times B_{1} \\
& A_{2} \times B_{2} \\
& A_{3} \times B_{3}
\end{aligned}
$$

But $A_{2}$ is the structural state of the organism after it has accomplished the function $\left(\mathrm{A}_{1} \times \mathrm{B}_{1}\right)$. The body, therefore, passes from the state $A_{1}$ to the state $A_{2}$ under the influence of the exterior conditions $B_{1}$, which have determined it to execute the function $\left(A_{1} \times B_{1}\right)$.

Thus the general biological problem comes back to this: what is $A_{2}$ with relation to $A_{1}$ influenced by circumstances $B_{1}$ determining the function $\left(A_{1} \times B_{1}\right)$.

1 This must not be taken as equivalent to the well-known formula of Herbert Spencer-the adjustment between internal and external relations-which, indeed, is a way of considering the phenomena, a descriptive method applicable to not-living matter as well as to living beings. The true definition is to be found in the result of function, in the law of functional assimilation, which is a theorem demonstrated by experience; the analysis into functions is an à priori method. 
We can already foresee the result of our investigation, since there is an aphorism summing it up ever since Lamarck. We have, indeed, been led by necessity of language to define the organ by the function; the same thing might have been done for a not-living mechanism. The formula "the function defines the organ" is therefore a general à priori formula, which has nothing to do with biology. But to the definition Lamarck added a verification-the expression of a fact of observation and experiment-when he said :

"The function creates the organ."

We may already divine under this very general formula the law we seek-the law which shall establish a relation between the successive states $A_{2}$ and $A_{1}$ of the organism, it being given that circumstances $B_{1}$ have determined in the individual the function $\left(A_{1} \times B_{1}\right)$. For a closer study of this fundamental law we must apply ourselves to the observation of cases in which-all things otherwise being the same-we shall be able to vary in the sum total B of circumstances exterior to the individual a factor or a group of factors easy to determine and even to measure. To the variation of this factor or of this group of factors we shall have to attribute the modification observed in the organism we are studying.

Before undertaking this essential study, we must bring out clearly an important result of our previous deductions. 


\section{CHAPTER XIII}

\section{LIFE IS THE RESULT OF STRUGGLE BETWEEN TWO FACTORS}

A Function, that is, in the language which we have adopted the activity of an organism at a given moment, may be represented by the symbolic formula:

\section{$\mathrm{A} \times \mathrm{B}$.}

The life of an individual being the succession of functions thus defined, we are obliged to say that, at each instant, life depends on two factors, one of which is the sum total of surrounding circumstances, the other the individual's actual structural state. In other words, no being bears life within itself. It transports with itself, wherever circumstances lead it, one of the factors of life, the factor A ; it meets every instant and everywhere the complementary factor B which determines in it, at each instant, the corresponding activity $(\mathrm{A} \times \mathrm{B})$. Its consequent state naturally depends on its antecedent state and on the phenomena of which it has since been the seat, that is, its state $A_{2}$ depends on $A_{1}$ and on $\left(A_{1} \times B_{1}\right)$.

Thus $B$ intervenes every instant to modify A; the series of factors $B$ determines the evolution $A_{1}, A_{2}, A_{3}$. . . But, while $A$ is modified under the influence of $B, B$ is also modified under the influence of A which, for example, consumes its oxygen, absorbs its radiations, and so on. Only, as $\mathrm{B}$ is not a living being its evolution $\mathrm{B}_{1}, \mathrm{~B}_{2}, \mathrm{~B}_{3}$. . does 
not concern us here; and moreover the intervention of $\mathrm{A}$ counts for very little in B's evolution.

However all this may be, the two factors $A$ and $B$ intervene every instant to modify each other reciprocally; and the phenomenon which brings them together-the life of $\mathrm{A}$ -must be considered as the struggle between these two factors.

We shall find this a handy expression ${ }^{1}$ in describing experiments in which we vary at will some measurable part of the sum total B. We say that we introduce a new enemy, or suppress a pre-existing enemy, in the struggle sustained by $A$ against exterior circumstances.

So long as A remains alive, we declare that it triumphs in. the struggle, even though it undergoes modification; and it is these modifications which we have to determine. The study of them will give us our great biological law.

It may seem odd that we should give this same name of enemy to substances which, like oxygen or food, are indispensable to the conservation of life in the individual, and to substances like poison, toxins, venoms, whose ordinary result is to induce death. But the interesting part of our researches is precisely to verify the generality of results obtained and to enable us to gather up into a single formula conclusions relating to the struggle with foods or against poisons, provided the individual survives. This will also enable us to extend the law which we discover to all variations of the factor $B$, even when such variations are too complex to be subjected to experiment.

1 This expression has already been employed in certain cases; we say, for example, that such a condition of the environment provokes such a reaction of a certain organism. 


\section{CHAPTER XIV}

\section{GENERAL APPLICATIONS OF THE NATURAL METHOD OF ANALYSIS}

THE formula established in the preceding chapter, namely, life is the result of a struggle between two factors, shows us at once where we shall find the natural method of investigation.

Indeed, we have already had occasion to say: when we have to study one object in reference to another object with which it has relations, our method of analysis of the first object must be such as to bring out precisely those of its elements which are in relation with the second.

In the present case, although life results from the struggle between the two factors which we call $\mathrm{A}$ and $\mathrm{B}$, we are forced to follow out the struggle in only one of the two-in the body of the living individual. We have therefore to study the modifications of A at each separate instant, but not at random. We ought to bring out clearly the relation of each modification of A to the corresponding modification of $\mathrm{B}$ that causes it. In other words, under exterior conditions $B$, when we vary experimentally some particular element, it is with reference to this particular element that we should study the modification obtained in A. In this way we shall arrive at simple laws.

Example: a healthy sheep is living in good hygienic conditions; into these conditions of life I introduce a new 
condition without changing anything in those already existing-I inoculate the animal with anthrax bacteridia-and I verify the struggle of the sheep against the bacteridia. Suppose the sheep is cured; no physiologist, clever as he may be, unless he has at his disposal a culture of anthrax bacteridia, will be able to find how the sheep that has been cured differs from the sheep before it was diseased. No direct chemical or physiological analysis of the modifications which have taken place in the sheep can be made in the present state of science. Let the observer be as conscientious as you please, while limited to our present processes of investigation he will have to assert that the sheep has not changed. And yet it has undergone a profound transformation, but it is with relation to the anthrax bacteridia. It has become refractory to the disease called anthrax; a new inoculation of the malady will not bring back the disease. We say it has acquired immunity with relation to anthrax bacteridia.

This example enables us to explain clearly the method which we are led to use. Into the exterior conditions B of the life of individual A we introduce a factor $b$; it is this factor $b$ which will henceforth have to serve us as a reagent in studying variations which its own influence determines in A. In this way we reach a simple law by a natural method, whereas if we had wished to analyse the variations of A without the help of $b$, we should run up against difficulties equivalent to impossibility.

This natural method of analysing facts has not yet, I believe, been proposed as a general method of investigation ; but it has already been applied, and fruitfully, in the field of the physico-chemical sciences. It has led up in particular to the Law of Lenz:

"The displacement of an electric current in the neighbour- 
hood of a closed circuit develops in it an induction current which tends to oppose itself to the displacement."

Also to the more general Law of Le Chatelier-a law which has been given a complete exposition in the admirable work of Willard Gibbs :

"In a system of bodies in the state of equilibrium, the modification produced by the variation of one of the factors of the equilibrium is of such nature that it tends to oppose itself to the variation determining it."

Here are two very general laws, both of extreme simplicity. They owe their simplicity to the fact that they have been established by the natural method which I propose and which we are now to apply in the domain of biology. Doing this, we are not guilty of novelty; long since the wisdom of the nations, without saying it in so many words, employed a method so fruitful in results by the proverb-Fit fabricando faber-that is, by often repeating an act you become fitter to do it again. The modification introduced into an organism by the repetition of a given operation cannot be analysed chemically. But it is very simple to take the operation itself as the reagent in the modification realized; through it we become fitter, habituated - to live is to habituate oneself. This is what the wisdom of the nations teaches us and in its inexhaustible treasure Lamarck found his law of habit_-"The function creates the organ." 


\section{CHAPTER XV}

\section{ANALYSIS BY MEANS OF REAGENTS OF THE SAME DIMENSION AS LIFE}

We will not start with the most general problem. That would consist in the study of the consequences to a living being of any variation whatsoever set up in the conditions of its life. We must begin by limiting ourselves to some very particular case in which the variation shows itself in a definite body carrying its own properties along with it just as chemical reagents do. We bring together an animal and a given reagent and, since all other conditions remain the same, we shall be able to assign to the reagent used and to this reagent alone the modifications we observe.

When we have a living being very low in organization, for example a unicellular being, we content ourselves with adding the given reagent to the liquid in which the being lives. I have already referred to an experiment of this kind : it consisted in adding a small quantity of phenic acid to the bouillon in which anthrax bacteridia were cultivated. But we estimated the result only by our first and artificial method of analysis; we verified that there was a variation with reference to a reagent other than phenic acid-the variation which we called attenuation of virulence. Such a result is not of general order ; it is only the expression of a particular fact.

When we have a living being of high organization such as 
man or any mammal, we introduce our reagent into that portion of the environment which is most directly in contact with the living substances of the given animal-that which we call the interior medium of the individual. In fact, we find in all these higher beings that the individual may be considered a closed sack traversed by a digestive tube-something like a lady's muff. This closed sack (Fig. 3) contains a colloid, in which bathe all the living elements of the cells; it is this not-living colloid which is called the interior medium.

In the relations existing between the living being and the environment, there are always two stages: 1. Exchange between the environing medium and the interior medium; 2. Exchange between the interior medium and the living elements. To avoid the first stage, which is itself a biological phenomenon, and to be undisturbed by the superposition of two

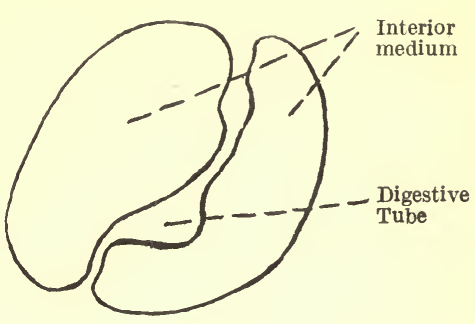

Fig. 3. different phenomena, we employ the method of subcutaneous injections. By breaking through the enclosing surface of the sack, we introduce our reagent directly into the interior medium of the individual. Then we wait for the effect of the reagent. It concerns us only when the individual survives the operation; it is the passage from the living state $A_{1}$ to the equally living state $A_{2}$ that particularly interests us. When the individual dies, biology has no longer to study it.

What we have said in the first part of this book with regard to the scale of phenomena makes us think that it will particularly interest our studies to use reagents of the 
same dimension as life. But what is the dimension of life? We know that vital phenomena are at once colloid and chemical; in our experiments, therefore, we shall have to use colloid reagents and purely chemical reagents; and we shall make still surer that we are using reagents really of the same dimension as life if we use living reagents.

The number of experiments made in the past twenty years by this method of hypodermic injection of well-determined reagents into living animals is something tremendous. It is safe to say that in no branch of science has like activity been shown; and the results have been in proportion. Even from the practical point of view this line of research has given us vaccination and serumtherapy-and birth to scientific medicine.

We have said that experiments should be made with chemicals, with colloids, and with living substances. The results obtained by means of the two latter groups of substances are by far the most important. Those due to the injection of a non-colloid solution of chemical substances, such as the injection of alkaloids or of chemically defined salts, concern us much less; they tell us nothing new and only verify the law of habit. This would seem to prove that, while life is at once colloid and chemical, it is, if I may say so, more accessible to experiment on the colloid side. We must, therefore, apply ourselves chiefly to experiments having to do with the injection of living or not-living colloids in order to establish the simple laws we seek. 


\section{CHAPTER XVI}

\section{PROTOPLASMIC FUNCTIONS OR FUNCTIONS OF COLLOID MECHANISM}

BY adopting this method of injection into the interior medium we evidently limit our studies to functions exercised in the interior of the tissues-to protoplasmic functions. It is not by shaking arms and legs that a man or an animal can struggle against a poison introduced into his blood. We shall have to see later whether laws discovered for protoplasmic functions apply equally to functions visible to the eye, like those which demand the help of the locomotor apparatus. We begin by the study of protoplasmic functions, because it is far easier and at once gives general results.

Whatever be the colloid substance injected, whether living or not-living, when the animal resists the injection without dying it seems not to have changed, that is, just so long as we do not use as verifying reagent precisely that colloid with which the experiment was made. Consequently, when the use of such a reagent is neglected, we might believe that the law of assimilation established in the third part of our book was rigorously exact: we should even be obliged to think that the animal, surviving as it does, has purely and simply assimilated, transformed into its own substance, the colloid which was injected into it and must have served it as a food. The variations therefore, if there are any, must be of little importance ; and indeed, from a 
certain point of view, the law of assimilation might seem exact enough.

It is no longer the same thing when we use the injected colloid as a reagent. Then we can always verify that the animal has undergone a modification with reference to the nature itself of the colloid, a modification which is so specific with reference to this colloid that it is commonly irresponsive to any other colloid.

A few examples are needed to establish so general a law, which seems, at first sight, to put aside and finally to condemn the law of assimilation pure and simple.

\section{Sheep Anthrax}

A first example may be taken from the history of the sheep cured of anthrax. This sheep, inoculated with anthrax bacteridia, was diseased (the period of struggle against the microbe we call disease). But, at last, after several days, all the bacteridia with which it had been inoculated disappeared from its interior medium. Now they had not been eliminated by the kidneys or other organs of excretion-care was taken in the laboratories to make sure of this. Therefore they were killed and assimilated by the sheep, for which they served as a food-a food not agreeable, dangerous even, but, in spite of all, a food, since the bacteridia were transformed into the substance of the sheep.

But after the cure, in spite of appearances, the sheep is not what it was before; it has become refractory to anthrax. If after a certain time you inoculate it again with anthrax bacteridia, it will not get sick as before; it will assimilate the bacteridia and not be troubled by them. Now it is only with reference to anthrax bacteridia that it has acquired such immunity. It is still liable to any other disease whatsoever that is peculiar to the species sheep. This fact may 
be summed up as follows : the sheep has become habituated to assimilating anthrax bacteridia; and this special habit expresses the modification wrought in the sheep by an injection of anthrax bacteridia, which it resisted.

Oftenest, if you select your sheep by chance and take the anthrax bacteridia from the body of a sheep that died of anthrax, the bacteridia with which the sheep is inoculated kill it after a few days; and then we can verify that the bacteridia have multiplied beyond measure in the interior medium of the sheep. In this case it is the bacteridia which, developing at the expense of the sheep's substance, assimilate the sheep.

Have they really and strictly assimilated the sheep's substance? Do they remain identically the same as they were at the start? For the most part we find that they too have been modified-and their modification relates to sheep. We say that they have increased their virulence for sheep. This means that they have become fitter to survive and prosper in a new sheep and to kill it. The only case in which they are not modified is when the bacteridia with which the inoculation is made already have the maximum virulence for sheep. In the same way, when the sheep wins in the struggle, it possibly suffers no modification, that is, if it already has the maximum immunity from anthrax. There is a limit to acquired habit and when the limit is reached, there is no longer room for further habituation. ${ }^{1}$ In both cases, when the sheep resists and when the bacteridia win the day, we are concerned only with the victor. It is only in the being which continues to live that we can establish the general law of habit.

The history of the struggle between anthrax and sheep

1 Then, as we shall see, the law of assimilation pure and simple becomes applicable. 
is interesting because it shows us systematically two antagonists, each of which, if we do not know their previous history, has unknown chances for victory. Our language will be still more symmetrical if we use for the sheep with reference to anthrax the expression we use for anthrax with reference to sheep : the refractory sheep is virulent to anthrax, or the virulent anthrax is refractory to sheepwhich is one and the same thing.

In this symmetrical struggle the side of the anthrax bacteridia is of more immediate interest to us ; first, because the bacteridia have not the histological complexity of the sheep and, consequently, our conclusions in regard to them take a more simple form; and then, because the bacteridia are in the sheep and for them the sheep realizes all the exterior conditions of existence. We have just noted that the life of a being $\mathrm{A}$ could be represented symbolically by the product of two factors $(A \times B)$. For the anthrax bacteridia, $B$ is the sheep.

On the contrary, when we study the sheep, we see that the anthrax with which it is inoculated is, indeed, one of the factors of its medium which play an important part in the life of the sheep (A) ; but it is only one of the factors. The factor $B$ comprises also atmospheric air, the grass of the field, the carnivorous wolf and so on. Even though attacked by anthrax, the sheep may be asphyxiated from lack of oxygen or may be eaten. The life of the sheep (A) is therefore not entirely determined by the fact of the presence of bacteridia in its interior, whereas the life of the bacteridia finds in the sheep which contains them all the elements of the factor B determining every instant their particular activity.

For the bacteridia, therefore, the function as defined is symbolically translated by the formula:

Bacteridia $\times$ Sheep. 
This protean mechanism-the bacteridium or any living cell which continues to live-directs all its activity against the exterior conditions of its environment. When it finds itself in the interior of a sheep, it directs its struggle against the sheep; it has become a definite mechanism-a bacteridium struggling against a sheep-and thus differs from that other definite mechanism-a bacteridium struggling against a phenicated bouillon.

Here we come to the delicate part of the exposition of this fundamental question.

Suppose some scholar endowed with knowledge wanting to us were able to verify completely, at any given moment, the entire structure, colloid and chemical, of a bacteridium considered precisely at that moment. The mechanism cannot be compared to that of a locomotive ; for the latter, when it works, works always the same. In this bacteridium, on the contrary, a great number of mechanisms may be verified, a great number of functions are possible. These functions are not determined beforehand in the structure of the bacteridium ; they can be determined only by the sum total $\mathrm{B}$ of the exterior circumstances which intervene in its working. In other words, take two anthrax bacteridia identically the same from every point of view at a given moment and with each of them I can realize a different mechanism by steeping the first in a phenicated bouillon and by inoculating a sheep with the second. In fact, the two mechanisms (Bacteridium $\times$ Phenicated Bouillon) and (Bacteridium $\times$ Sheep) have descendants that differ. Those of the phenicated bouillon undergo certain variations which we can scarcely appreciate directly, since the bacteridium never habituates itself completely to the phenicated bouillon; but the variations, if we stop the experiment soon enough, show themselves by a diminution of virulence 
for sheep. ${ }^{1}$ On the contrary, the bacteridia of the sheep, if they triumph, acquire an increase of virulence.

As we do not know the structure of a bacteridium, we may imagine provisionally that it comprises different active parts and that these parts arrange themselves differently in case of a new struggle, so as to realize a mechanism fitted to the struggle; or again that, of the different constituent parts of the bacteridium, some do more than others to realize the mechanism made necessary by the sum total $\mathrm{B}$ of exterior conditions. If it were not a fault in method, we might compare the bacteridium, a simple being, with man, a complex being. When man performs an act or exercises some function determined by circumstances, the man's different parts do not intervene equally in the execution of the act. To fix our ideas, we may suppose that the same thing takes place in a bacteridium $\mathrm{A}$ which the sum of circumstances $B$ leads to exercise the function $(A \times B)$.

Without further reference to such hypotheses, we can verify that, under the prolonged influence of a sum of circumstances determining in the bacteridia the function (Bacteridia $\times$ Sheep), there appears in the conquered sheep a race of bacteridia which have acquired exactly the character corresponding to the execution of the function, namely, resistance to the sheep.

We have named organ all those parts taken together which work in union with each other in the exercise of a function. The total function of the bacteridia enclosed within the sheep is determined by the nature of the sheep. So we can consider, at each successive instant, the actual mechanism of the parasite bacteridium as the organ of the struggle against the sheep. We also verify, after a certain number of genera-

1 Also by the loss of the faculty of giving off spores. 
tions when the sheep has died from the attack of the multiplied bacterıdia, that all these bacteridia have in the highest degree precisely the aptitude to struggle against sheep. In other words, that which multiplied in the sheep was bacteridian organs of struggle against the sheep. There was, indeed, an assimilation of the sheep's substance by the bacteridia, but it was not any and every assimilation. The bacteridium's assimilation in the sheep does not give the same results as the same bacteridium's assimilation in phenicated bouillon; the assimilation is relative to the function performed or, more briefly, it is functional assimilation.

This is the great biological law : it is the literal translation of Lamarck's principle "The function creates the organ." Indeed, the function defines the organ as we have seen; and if circumstances are such that the same function is exercised for a long time, the corresponding functional assimilation transforms the given organism into the organ itself of the function.

We have noted that habit has limits; beyond a certain degree no individual can acquire habituation to given conditions. This means that the parts of the mechanism which are less useful in executing a function long repeated are not on that account completely destroyed; enough remains for other functions to be possible. No individual ever adapts itself so exclusively to one kind of life as to become incapable of all others. ${ }^{1}$

An experiment of Pasteur, Chamberland, and Roux, is of great interest to us in this connexion.

1 There are, however, parasites very strictly adapted to their hosts-for example, the parasite of malaria in man's blood. But even this parasite, delicate as it is in its needs, adapts itself to the conditions found in the body of mosquitoes of the genus Anopheles. 
By artificial means, such as culture in phenicated bouillon, they obtained anthrax bacteridia which no longer have the slightest virulence for sheep, that is, bacteridia which, if any sheep were inoculated with them, would be assimilated without rendering the sheep diseased. But the organ of resistance to the sheep was not on that account destroyed in these bacteridia. A favourable occasion came to show it. It happens with anthrax bacteridia that the organ of resistance to sheep is defined in the same way as the organ of resistance to the rabbit, guinea-pig or mouse. The least virulent bacteridium for a sheep, if it lives at all, has always been a sufficient organ to struggle at an advantage against a young mouse a day old; by the time it has killed this first mouse it has become capable of killing a mouse a week old; and, having killed this mouse a week old in its turn, it will kill an adult mouse, and then a guinea-pig-and then a sheep. But you must give it time to recuperate its atrophied qualities. It is more difficult for a man who has worked with his brain twenty years to become a blacksmith or for a blacksmith to become a professional thinker; but it is not impossible. The exaggerated development of one organ in an individual does not totally destroy the constituent elements of other organs. Between individuals of the same species, even when they are subjected to different regimens, there are never other than quantitative differences: all have the same parts, only more or less developed in each. We shall see this even in the definition of species in biology.

\section{Vaccination}

On account of the practical interest of the matter, I note the fact that Pasteur proved, from such phenomena as we have been studying, the general method of vaccination. 
By empirical processes we can determine in a pathogenic microbe a modification causing a diminution of virulence with respect to a given species; by inoculating an animal of this species with an attenuated microbe, we produce in it a light form of disease, of which it is easily cured; the animal comes out hardened and resists a second inoculation from a more virulent microbe. Finally it becomes refractory to the attacks of the most virulent individuals of the pathogenic species.

When the sheep has been cured of anthrax we can say of it what we said of the anthrax when it triumphed over the sheep ; it has developed within itself the organ of resistance to anthrax by functional assimilation. But since at the same time it has breathed and grazed and excreted, it has also developed the other parts of its organism along with the mechanism of resistance to anthrax. The case of the sheep is less simple than that of the anthrax; but once the latter is understood, the former becomes easily intelligible. To speak with rigorous exactness, as we have already noted, we ought not to separate the organ of resistance to anthrax from that of respiration, mastication or excretion; if one of these partial functions should happen to fail, the others also would cease. In the very general language we are now using, we ought to speak solely of a function of the whole-the function sheep-which in the present case comprises resistance to anthrax. The division of physiological labour will come up for study later.

\section{Dead Colloids, Toxins, Foods}

Instead of injecting into an animal a living being like the anthrax bacteridium, we may try what will happen if we introduce into the animal's interior medium some dead substance. 
The results thus obtained from purely chemical, noncolloid substances are of little interest; yet we know that men accustom themselves to increasing doses of morphine and that Mithridates reached a point where he swallowed with impunity large quantities of different poisons. The introduction of colloids taken from other living beings -for example, elements from other animals (milk, blood, liver, etc.) or filtered microbe cultures (toxins, diastases)-is infinitely more instructive.

As only one of the two antagonists in this case is living, we occupy ourselves only with an animal that survives the injection. At the end of a certain time all the injected colloid has disappeared, assimilated by the living animal which uses it as a food. The animal, after assimilating the colloid, has apparently undergone no modification; and we might think that it was all the same to feed the animal this or that colloid, provided it did not die.

Nevertheless, when the injected colloid is what we call a toxin or a venom, we observe a modification in the animal into which it has been injected. The words toxin, or venom, are expressions altogether relative ; if they apply to the species of animal studied, it is for this reason-the injection of such substances, in sufficient quantity, sickens or slays a normal individual of the species. Suppose the animal does not die; when cured it will be able to receive a new injection of the same toxin or venom without sickening again, at least not so much as before. Finally, it may become refractory to the toxin or venom and support very large doses without suffering from it.

Here, again, there has been functional assimilationthe development of the organ defined by the function of resistance to the venom or toxin. 
Instead of venom or toxin, let us inject into an animal quite harmless substances like milk, calf's blood or beef liver. To all appearances we shall have no means of knowing if the animal has been modified by the injection. If not disturbed at all by the first injection, it cannot be less disturbed by the second. And yet it is hard to say that a colloid substance can be quite harmless; injected in sufficient doses, it will clearly kill the patient-by indigestion. But the symptoms of uneasiness after the injection of a reasonable dose are so slight that it is impossible to observe a diminution of them in the second injection.

Luckily, at least in certain cases, a very important peculiarity allows us to verify indirectly a variation of the organism even with colloids which seem quite harmless. This remarkable peculiarity has to be studied in the next chapter; it is the starting point of a fruitful method known for some years back under the name of serumtherapy.

First, there is an observation useful to make.

Habit has limits, as we explained. Once an animal is habituated to the maximum under given conditions, it is able to undergo these conditions without experiencing henceforward any variation. When, therefore, a living being has been long subjected to circumstances which have not varied, the law of functional assimilation gives way to the rigorously exact law of assimilation, which we verified as an approximate law in the third part of this book. This is how we were able to verify assimilation without variation in an anthrax bacteridium watched by a skilful experimenter in its bouillon. We might say the same thing of a bacteridium which has already killed several sheep and consequently acquired the maximum virulence for sheep, should it multiply without variation in a new sheep. The law of functional assimilation is relative to the periods of change 


\section{THE NATURE AND ORIGIN OF LIFE}

in the circumstances; it is consequently very general-for circumstances ordinarily change in nature. But if, during such periods, the law of assimilation is only approximate, it is rigorously exact whenever conditions are realized for a long time and remain constant. 


\section{CHAPTER XVII}

\section{CASES IN WHICH THE RESULTS OF FUNCTIONAL ANALYSIS ARE TRANSPORTABLE OUT OF THE BODY-SERUMTHERAPY}

So far function, as we have defined it for the needs of the analysis of biological phenomena, seems only a convenient manner of speech. But an altogether unexpected discovery, which is bound to have vast influence in science, shows cases where the decomposition into functions is something more than a form of words.

Take, for example, a rabbit, into whose peritoneum has been injected cow's milk; the milk is assimilated and the rabbit is none the worse for it. Yet he has undergone a change with respect to cow's milk-a fundamental observation made by $J$. Bordet. The serum of this rabbit which has assimilated cow's milk will henceforward give in vitro a precipitate with cow's milk and with cow's milk only.

Thus we are sure of two things. The injection of cow's milk into a rabbit gives the rabbit a specific modification with regard to cow's milk-and this we might foresee by analogy with injections of toxins and venoms. Secondly, a result of this specific modification can be transported in vitro with the serum, which is a dead colloid extracted from the rabbit. The function (Rabbit $\times$ Cow's Milk) is no longer a mere matter of words; we can carry about its result in a bottle in the form of a serum; and this serum enables 
us, in particular, to distinguish cow's milk from all other milks. The specific serum thus obtained gives a precipitate neither with asses' nor woman's nor sow's milk.

In like manner inject into the peritoneum of a guineapig red blood globules from a goose. We find that these globules are little by little digested and assimilated by the guinea-pig, and that henceforward the guinea-pig's serum has the property of dissolving in vitro the blood globules of the goose.

This result has been generalized for a great number of tissues. Each of them, when assimilated by a given animal, develops in the animal's blood a transportable quality which is specific in relation to the tissue assimilated.

We can understand all the practical importance of such a discovery; it contains all serumtherapy in itself. If you habituate a horse to assimilating diphtheritic toxin, its serum will be capable of destroying the toxin in a child attacked by the terrible malady, provided the serum of the horse is so prepared as not to be itself a poison to the child. This is the general method discovered by Behring and Kitasato, and made applicable by the efforts of Emile Roux.

To tell the truth, we already knew of an analogous transportability of functional properties by dead colloids. A filtered culture of anthrax bacteridia, if a sheep were inoculated with a sufficient quantity of it, might bring on fatal disease with symptoms analogous to those of bacteridian anthrax. But at the beginning this result of observation was not interpreted as it is now. It was thought that, in the disease caused by anthrax, the excrementitial substances produced by the bacteridia played the principal part-an opinion that will also have to be discussed later.

In fact, when we filter a culture bouillon of anthrax 
bacteridia, we obtain the result of the following function (Bacteridium $\times$ Bouillon) and not that of the function (Bacteridium $\times$ Sheep). The fact that this filtered liquid is nevertheless harmful to the sheep proves that the two functions do not differ greatly from each other. We have already seen that the functions (Bacteridium $\times$ Mouse), (Bacteridium $\times$ Guinea-pig) and (Bacteridium $\times$ Sheep) are all of the same order. The bacteridium has not specific actions well characterized.

It is quite different with another microbe which is also well-known-the rouget of the hog. The function (Rouget $\times$ Rabbit) antagonizes the function (Rouget $\times$ Pigeon), since the rouget's habituation to killing the rabbit diminishes in virulence with the pigeon.

Whatever their importance in scientific medicine, such facts of transportability of functional results in dead liquids have far greater importance in biology.

Suppose that to each function clearly defined in a living species there should correspond a specific functional activity transportable in a dead colloid. We should then be able to preserve in labelled jars every possible function of a living being in the form of colloids able to perform, at least in part, each of these specific functions. That would be a real analysis of the vital activity of the species studied. Such an analysis might be more or less complete, but it would be indeed real.

One of the first results obtained along this line was the fabrication of alcohol in a sugared must by means of dead colloids furnished by yeasts. This occasioned the assertion that such dead colloids are partially alive-which, at least in this form, seems absurd. What is true is that we can decompose a greater or less part of the vital activity of a living being, and perhaps all its activity, into partial activi- 
ties transportable in dead colloids; and this is enough to prove that vital phenomena are not essentially different from other natural phenomena. Only we must take as living only that body which has all the attributes of a living being.

\section{Diastases and Excrementitial Substances: Physical Assimilation or Digestion}

The foregoing example-alcohol fabricated in a sugared must by means of a dead colloid coming from a yeastbrings up at once a question that seriously complicates the method of analysis by decomposing the total vital activity into partial activities transportable in colloids.

At one and the same time two processes are going onassimilation by a living substance, either in the strict sense in a constant medium, or functional assimilation or adaptation under new conditions of the medium ; and the production of accessory substances of the assimilation. The latter are oftenest called excrementitial; they are indicated by the letter $\mathrm{R}$ in the equation of elementary life as manifested (p. 49). They are chemically defined substances and are produced always the same just so long as the conditions of life in the species studied remain the same. Such, for example, is alcohol, an accessory to the assimilation of sguared must by beer yeast.

There is not the slightest comparison possible between this excrementitial substance alcohol and the dead colloid which, extracted from a culture of beer yeast, is able to produce the alcohol in a sugared must.

Speaking generally, diastases or enzymes are the dead colloids able to transport with themselves a part of the vital activity of a living being. The diastases we know only by their effects; and these are precisely the expression of the 
living being under observation relatively to the substance on which the diastases act. Now they are mixed in the culture bouillon with excrementitial substances (substances $R)$; and so, when we study the effect of a filtered culture ${ }^{1}$ of a microbe or yeast on an animal or any reagent whatever, we cannot distinguish what is due to the diastase and what to the excrementitial substance. This is a hindrance in the study of the diastases.

As we have said, the diastases are dead colloids. Probably their activity is due to their peculiar colloid state; and this in fact, manifests itself for the most part by variations introduced into the colloid state of other substances. We know that, secondarily to these colloid variations, chemical reactions resulting from them must often be produced; but that does not prevent our believing the action of the diastase to be of colloid order, even though we can verify only the secondary result, which is chemical.

Sucrase is an example; it is a diastase produced by yeasts and other moulds; and it has the property of inverting cane sugar. This simply proves that the solution of cane sugar, as a chemically defined compound or saccharose, is stable only in a colloid state differing from that of sucrase. There is a relation of equilibrium between the chemical and physical states of this particular substance.

This idea of the purely colloid action of diastases suggests a general interpretation of their origin. A body living in a must, bouillon, or any other colloid, multiplies by assimilating the medium, that is, by fabricating at the medium's

1 Filtered or centrifugated; for certain diastases do not pass through the filters, and so it is preferable to separate the bodies of the microbes by means of an apparatus acting centrifugally like that used in removing the cream from milk. 
expense protoplasm identical with its own. In such assimilation there are two distinct phenomena. The protoplasm fabricated is in reality identical with that which produces it, both from the chemical point of view and from that of the colloid or physical state. There are therefore in reality two assimilations. One of them may be called physical or colloid assimilation; it consists in setting up an equilibrium between the colloid state of the living body and that of the substances of its medium with which it is struggling. The second is assimilation properly so called, or definitive, and is probably subordinate to the first; but it takes place only within the limits of the living body itself, within its protoplasm, in a word. Physical assimilation, on the other hand, when more or less advanced, may trench on the surrounding medium.

Thus what we call secretion of sucrase by yeast is only the progressive influence of the colloid state of the function (Yeast $\times$ Saccharose) on the medium containing the saccharose. The different diastases secreted in a medium containing various active substances by a living element $A$ must be colloid verifications of the partial functions $\left(A \times B_{1}\right),(A \times$ $B_{2}$ ), in which $B_{1} B_{2}$, . . , are the different active substances whose sum constitutes the totality $\mathrm{B}$ of exterior conditions. To separate such diastases one from another amounts, therefore, to analysing the total function $(A \times B)$ into its partial elements $\left(\mathrm{A} \times \mathrm{B}_{1}\right),\left(\mathrm{A} \times \mathrm{B}_{2}\right), \ldots$. constituting an effective analysis of the total vital activity of the species $A$ under conditions $B$.

This remarkable physical assimilation has long been known incertain particular cases under the name of digestion; but, as the properties of colloid bodies were unknown, digestions were commonly looked on as dissolutions. For example, meat digested by gastric juice was looked on as dis- 
solved $^{1}$, whereas in reality it simply passed from one colloid state to another colloid state in equilibrium with that of the stomach cells. This new way of looking at things explains quite naturally a paradox that has been the occasion of many an odd explanation.

"Why does not the gastric juice digest the walls of the stomach?"

The answer is direct:

"Because the gastric juice's work is to bring the colloid states of the ingesta into equilibrium with the stomach cells, whereas the stomach cells are naturally in equilibrium with themselves."

In the same way astonishment is felt that the venom of scorpions and serpents should be inoffensive to themselves. The notion of physical assimilation explains it all.

Physical assimilation, in certain cases, is carried on both inside and outside the living protoplasm; but it is not wonderful that, for the mostpart, it should be much stronger inside the active cells and be exercised in the surrounding medium with an intensity decreasing in proportion to the distance from the centre of activity. For this reason we are obliged in many cases, in order to procure certain diastases, to triturate mechanically the cells which have produced them. It is also possible that certain activities should become localized inside the living cells and have no diffusion outside; relatively to such activities our effective functional analysis would be incomplete.

The history of physical assimilation is very clearly summed up in the phenomena which take place in an amoeba vacuole (Fig. 4). The amoeba, which comes up for study again with

1 When milk is digested by rennet its physical assimilation is made known by a more solid state (curdled milk), and not by a more liquid state. 


\section{THE NATURE AND ORIGIN OF LIFE}

relation to movement, is a little protoplasmic mass of variable form enclosing foreign bodies within its own substance in a vacuole containing water. Suppose the ingested body to be a living body. Every living body has its own personal colloid or protoplasmic state, which is the condition sine qua non of its life. In the present instance, therefore, the physical equilibrium of the liquid in the vacuole has to undergo two antagonistic strains from two different protoplasms, of which one is enclosed within it while the other surrounds it. One of the two wins the day; the other dies and is physically assimilated or digested by the first. Here is a struggle between two protoplasmic activities through the intermediary of an interposed liquid, the liquid of the vacuole. When the amoeba carries the day, we say that the vacuole, having filled itself with the

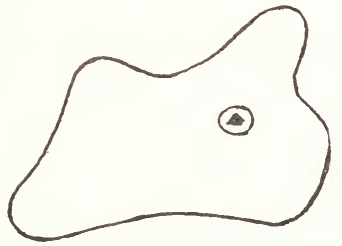

FIG. 4. amoeba's digestive juices, has digested and assimilated the ingested animal.

We can narrate in the same way the history of a bacteridium injected into a sheep ; only here the interior medium of the sheep takes the place of the amoeba's vacuole. 'The thing is to know whether the bacteridium will assimilate the sheep or the sheep assimilate the bacteridium. But, once the struggle is over, it is plain that the interior medium of the sheep will contain the transportable activity which won the day. As we have seen, this is the origin of serumtherapy.

The same story may be told when the ingested or injected body is a dead colloid. Such a colloid, inasmuch as it is a definite colloid, owes its existence to its particular physical state. The thing is to know whether the injected colloid will win the day over the interior medium of the animal, on 
which it will impose its own state, or whether it will be vanquished and assimilated by the animal. In the former case we say the colloid is toxic ; in the latter that it is a food in relation to the given animal. When the animal A conquers, the colloid $\mathrm{B}$ must have determined in the animal the function $(\mathrm{A} \times \mathrm{B})$, the trace of which will be found in its serum ; and the animal will consequently develop the organ corresponding to the function. In this way, when the battle is over and the colloid B has been conquered, the animal A will continue to produce in its interior medium the diastase defined by the function $(\mathrm{A} \times \mathrm{B})$. This, indeed, is currently verified. A horse into which diphtheritic toxin has been injected continues for a long time to furnish an antitoxic serum; and when the function weakens it is revived by giving the animal a new injection of the same toxin. 


\section{CHAPTER XVIII}

\section{COLLOID STATES AND PHYSIOLOGICAL DIVISION OF LABOUR}

WE have been making great use of the expression " colloid state," and we are unable to define it. At least, in the present state of science, we cannot describe the arrangement of particles and solvent in a given colloid, nor the electric state of the parts, nor establish by figures the characteristics of any particular colloid state. In other words, we cannot make an artificial analysis of a given colloid to satisfy the human mind. But on the other hand - and this precisely is the natural method of biological studies-we can characterize a colloid with reference to another colloid.

We recognize a colloid by its origin and its effects. For us it is defined by the function $(\mathrm{A} \times \mathrm{B})$, in which $\mathrm{A}$ and $B$ are known. For example, we defined sucrase by the function (Yeast $\times$ Saccharose) or (Aspergillus $\times$ Saccharose), and we recognize it by its inverting cane sugar. Perhaps even we are wrong in saying Sucrase simply, defining it by its effect, for it is probable that the Sucrase of yeast is different from the Sucrase of Aspergillus. When the colloid is a toxin we have a contrary tendency to define it solely by its origin and to say, for example, "tetanic toxin," whereas the function (Tetanus $\times$ Sheep) is perhaps different from the function (Tetanus $\times$ Guinea-pig). 
However all this may be, we recognize one colloid by its action on another colloid; and most of the time we can verify directly that colloids follow a determined order according to the foreseen result of their struggles, one against another.

For example, the rennet of the calf's stomach curdles milk (the rennet's victory imposing its own colloid state on the milk); gastric juice digests meat (victory of the gastric juice); and so on.

In some cases the colloid used as a reagent is a living animal ; tetanic toxin kills man by producing in him certain characteristic lesions. We can even say there is no colloid reagent as sensitive and exact as living beings.

But all these colloids which we call diastases or toxins, when compared with the colloids which they are capable of conquering by imposing their own physical state, have to be considered as foods in relation to the animals which digest them. Rennet injected into a rabbit is assimilated by the rabbit, which develops the function (Rabbit $x$ Rennet), and the result is to produce in the rabbit such a modification that its serum will henceforth annihilate in vitro the effect of rennet on milk. There are certain yeasts that feed perfectly well on tetanic toxin. A colloid, therefore, cannot be taken absolutely either as a toxin, a diastase or a food: it is diastase for certain colloids and food for others. Finally, there are other colloids indifferent to each other, that is, capable of existing together without reciprocal influence.

All the facts which we have established since we began studying living beings by the natural and genuinely biological method of investigation prove what I asserted at the start. While vital phenomena bestride the chemistry and physics of colloids, they are most accessible on the colloid side. 
Chemical actions between two colloid bodies take place for the most part indirectly, by a first colloid battle ; and its results are realized in a definitive colloid equilibrium and henceforward direct the chemical reactions attached to such an equilibrium of the colloids. Serumtherapy (and nearly all pathology) consists in phenomena of colloid struggles.

Two facts chosen among a thousand others prove the importance of colloid phenomena with relation to purely chemical reactions in the field of biology.

1. If you inject into animals increasing doses of the soluble alkaloid morphine, you produce in the animals the habit of the poison; but the serum of morphinized animals does not carry with it in vitro any anti-morphine property. The morphine being really dissolved, there was at first no colloid equilibrium between it and the living being. The phenomenon of habit must have been produced only in the midst of the tissues; probably it was a directly chemical phenomenon. The explanation of this particular case will occupy us again.

2. Two living beings, differing greatly in species and consequently having very different chemical structures, may nevertheless be in colloid equilibrium. The serum resulting from the function (Mammal $\times$ Serpent's venom) may be utilized against tetanic toxin.

Facts of the same order explain why the hedgehog is refractory to the bite of vipers, and so on.

\section{The Division of Labour}

The language we have been using authorizes us in speaking of the division of labour, even with the simplest living beings like bacteria or yeasts. Synthetically, we have 
verified that the activity of a living body $\mathrm{A}$ in the sum total of circumstances $B$ can be summed up in the function represented by the symbolic formula $(\mathrm{A} \times \mathrm{B})$.

But if we are able to decompose the sum total $B$ into several terms, $B_{1}, B_{2}, B_{3}, \ldots$, we can analyse the total function $(\mathrm{A} \times \mathrm{B})$ and decompose it into partial functions $\left(A \times B_{1}\right),\left(A \times B_{2}\right),\left(A \times B_{3}\right), \ldots$ We know, of course, that when we make such an analysis we risk being incomplete, for we may neglect important phenomena resulting from the rebound of each of these partial functions on the others.

Nevertheless-especially when a diastase that can be isolated corresponds with each partial function $\left(A \times B_{1}\right)$, $\left(\mathrm{A} \times \mathrm{B}_{2}\right), \ldots$ - . we find a great advantage in using this artificial analysis.

Take the case in which the factors $B_{1}, B_{2}, B_{3}$, are colloids transportable with their properties. According as we bring into conflict with $\mathrm{A}$ a dominating quantity of one or other of these substances- $B_{1}$, for example-we shall define and develop by functional assimilation what we should call the organ corresponding to the function $\left(\mathrm{A} \times \mathrm{B}_{1}\right)$. The result would be the appearance of the diastase of this function in the surrounding medium and the production of the diastase would continue - the organ which fabricatesit being developed - even when we cease introducing the factor $B_{1}$ into the medium.

When we have studied as completely as possible the species A with reference to all the substances which may enter into conflict with it, we say, for example-this species is capable of secreting three well-defined diastases, one that digests fats, another that digests sugars, and another that digests albumens. And in any new functioning of this living being A with relation to some complex substance we 


\section{THE NATURE AND ORIGIN OF LIFE}

shall be able to find diastasic secretions of these three kinds, in proportions varying according to the case.

For so very simple an example, the division of labour will be reduced to the possibility of an exact definition of three organs corresponding with three well-determined functions.

But let us suppose that, in a living body of large dimensions (Fig. 5) the form itself of the body brings habitually into conflict with the different enemies $\mathrm{B}_{1}, \mathrm{~B}_{2}, \mathrm{~B}_{3}$, the regions $\mathrm{M}, \mathrm{N}, \mathrm{O}$ of the body; then the function $\left(\mathrm{A} \times \mathrm{B}_{1}\right)$, defined at the point $\mathrm{M}$, will develop more particularly at that point the corresponding diastasic secretion.

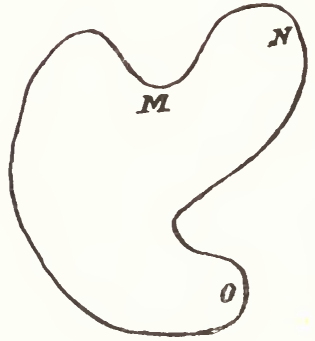

Fra. 5. There will be, to a certain degree, localization of functions and, if it lasts long, the points $\mathrm{M}, \mathrm{N}$ and $\mathrm{O}$ of the body will end by acquiring particular characters in relation with the particular conditions to which they are subjected. Functional assimilation will develop at each of these points parts of the mechanism which will result in producing precisely the necessary diastases; the body of the individual will become, by the fact, heterogeneous; and the division of labour may become anatomically verifiable, for the colloid states of living substances influence their morphology.

When, instead of a continuous protoplasmic mass (which our reasoning in no wise supposes), the organism of Fig. 5 is made up of an agglomeration of cells, then the differences realized by the conditions at points $\mathrm{M}, \mathrm{N}, \mathrm{O}$ of the body will manifest themselves by different aspects of the cells placed at those points. The division of labour will create histological differentiation. 
How far can such differentiation go ? We have already said that habit has limits. Ought we to think that each cell of a body comes to a point of differentiation where it is capable of but one function exclusively-the function $\left(\mathrm{A} \times \mathrm{B}_{1}\right)$, for example? Or ought we, on the contrary, to believe that differentiation is limited to the development, in each cell, of certain organs rather than others which still exist there, although now of little importance? This is a disputed question.

The chicken comes from an egg which has neither muscles, nor nerves, nor bones; but we have an idea that functional assimilation develops differently for each tissue certain peculiarities, all of which existed in the egg. In other words, the egg is somewhat muscle, somewhat nerve, somewhat bone ; the question is to know whether muscle, nerve, bone are also somewhat egg, that is, whether, along with the organ corresponding to their special function, they contain rudiments of organs corresponding to the functions of other tissues.

However this may be, the law of functional assimilation, from the start, prevents us from being astonished at one thing, namely, that in so complicated a mechanism as man each tissue should be exactly adapted to the needs of the place it occupies. The law of functional assimilation has been established for cases in which exterior circumstances $\mathrm{B}$ are reduced to transportable colloid elements-and this limits us here to the functions of protoplasmic mechanism. But it is easier for us, after what we have learned, to extend this general law to functions of the mechanism as a whole. 


\section{CHAPTER XIX}

\section{FUNCTIONS OF THE MECHANISM AS A WHOLE}

Ат the beginning of the fourth part of our work, we established the fact that the decomposition into functions of the total activity of a complex individual like a man or dog may be made in any number of ways as the observer wishes. Such functions, thus fancifully defined, have no other than a descriptive interest. Moreover, their definition is itself a danger, for the sum of all these functions separately defined will not represent the total activity of the individual analysed unless we take into account the inevitable relations which unite each of the partial functions to all the others.

Nevertheless, we have drawn a certain advantage from considering functions of protoplasmic mechanism as defined, each on its own account, by a factor taken by itself in the environing circumstances. This has enabled us to bring under a general formula the numerous experimental facts gathered during the past twenty years in laboratories of experimental pathology.

The animal organism can be considered as a mechanism from several points of view, according to several scales of dimension. There pass in it chemical phenomena and colloid phenomena; and we know that the colloid or protoplasmic mechanism has its influence on the chemical 
mechanism. Reciprocally, the chemical mechanism has its rebound in the colloid mechanism. Analogous relations exist between the mechanism as a whole and the colloid or protoplasmic mechanism. For example, movements of locomotion, which are the easiest to observe among the movements of the mechanism as a whole, are attached by relations of cause and effect to the protoplasmic state of the tissues which compose the limbs. The movement of arms or legs is inseparable from muscular contractions and nervous currents, which are functions of protoplasmic mechanism. In the functions of the mechanism as a whole we shall, therefore, as before have to consider the corresponding functions of protoplasmic mechanism; and it is very certain that, here as there, the exterior circumstances will determine the particular activity of the organism. The only difference is that, instead of acting directly on the protoplasmic mechanism as did the colloid factors $B_{1}, B_{2}$, $\mathrm{B}_{3}, \ldots$, the external factors of the action under consideration act on the colloid mechanism only through the intermediary of the whole mechanism, which belongs to a higher scale.

Example.-There is a bowl of milk on the table. If I inject the contents of the bowl of milk into the peritoneum of a guinea-pig, I determine directly in the guinea-pig's protoplasm the function (Guinea-pig protoplasm $\times$ Milk) -a function of protoplasmic mechanism. But a cat arrives before the bowl of milk. The odour arising from it, the light issuing from it, influence the olfactory and visual organs of the cat. The sensorial impressions thus created (by a protoplasmic mechanism which will have to be studied later) transmit a modification to the colloid state of the nerve cells of the brain. This modification is distributed in the different nerve centres along the lines of least resist- 
ance existing in these centres at the given moment; and it ends along the centrifugal nerves in a great number of locomotor muscles which contract successively. As a result of all this, the cat approaches the bowl of milk, lowers its head and begins lapping up the contents.

Here we have something of infinite complexity-an activity which it is very difficult to analyse as a whole and which was determined in the cat by the bowl of milk. We have still to observe that the bowl of milk is not alone in this determination; the state of the surroundings and the state of the cat both play an important part. If the cat had just been fed or saw some one near the bowl of milk armed with a stick, the movements determined would have been quite different.

In any case, we are forced to verify the fact that the whole cat takes part in the execution of the function we are considering. We cannot assign to the exercise of such a function any organ that does not include the whole cat. The function studied is a page in the history of the cat's activity ; the corresponding organ is the corresponding page in the history of the cat's structural evolution. In the cat, at the given moment, there is a well-defined mechanism different from that which was defined there a moment previous and accomplishing a different thing. In other and rigorously exact language, the living cat is a succession of different organs, all of which we call by the same name "cat" and each of which is a mechanism apart.

Returning to our notation, we can say that the cat, being a succession of organs $A_{1}, A_{2}, A_{3}, \ldots$, exercises successively in conditions $B_{1}, B_{2}, B_{3}$, the successive functions $\left(A_{1} \times B_{1}\right),\left(A_{2} \times B_{2}\right),\left(A_{3} \times B_{3}\right), \ldots$ And the accomplishing of the function $\left(A_{1} \times B_{1}\right)$ transforms cat $A_{1}$ into cat $A_{2}$, which straightway executes the function $\left(A_{2} \times B_{2}\right)$ 
and so on indefinitely as long as there is life-for Biology has no Statics.

The only relation which direct observation enables us to verify between the cat's successive states $A_{1}$ and $A_{2}$ is this : when the cat often exercises the function $(A \times B)$, it becomes fitter to exercise the same function again. Always the same law of habit which Lamarck took from the wisdom of the nations-the function defines the organ; the function often repeated creates the organ.

Let us take this observation into the field of colloid mechanism. If at a point $M$ of the body a protoplasmic mass is made to execute several times in succession the same operation of the mechanism as a whole, we understand that it will be so modified by the operation itself as to become fitter to exercise the same operation afterwards. The case may be compared with that of protoplasmic elements struggling against a determined toxin or food; only, in the present case, the special function of the protoplasmic mass is defined indirectly by the whole mechanism of which it is a part.

But in the adult animal protoplasmic masses localized at different parts of the body are not subject, in their relations with the whole mechanism, to such wide variation as in their relations with toxins or foods. ${ }^{1}$ We have seen the same protoplasm successively fabricate antitetanus, antidiphtheria, etc., that is, in each case carry on a specific struggle against a new enemy while acquiring a new organ. It is not the same thing in the case of protoplasmic masses considered as constituent elements

1 It is true that, according to M. Metchnikoff, it is not the fixed or constructive tissues which carry on the antitoxic defence, but rather migratory elements, or phagocytes, having nothing to do with the mechanism of the animal as a whole. 


\section{THE NATURE AND ORIGIN OF LIFE}

of the whole mechanism; each element, situated at a fixed point of the adult mechanism, can execute only one kind of protoplasmic function. Muscle is muscle, nerve is nerve, and we never find a muscle transforming itself into a nerve. Here, then, there is no longer question of variation in the nature of the mechanism of each cell, but simply of a more or less considerable functioning of the cell according to a model traced beforehand in each element as histologically defined. In other words, a given element situated at the point $\mathrm{M}$ of the body always does the same thing, no matter what may be the function of the mechanism as a whole determined in the animal by circumstances. Only, this same thing which it can do it does a great deal, or little, or not at all, according to the manner in which the organ that the animal is at the given moment happens to be defined.

Besides, we do not know if the elements of tissues under observation here and now in an adult animal have come to the absolute term of their differential evolution. We said a little while ago that we never know whether to-day's muscle is not still a trifle egg, that is, whether it does not still contain rudiments of functions which are characteristic of other tissues. If this were so, we ought to expect an application of the law of functional assimilation as previously established-by dint of acting as a muscle (by muscle-ing) the muscle becomes more muscle. In any case we should remember that this happens in the evolution of species which, by progressive adaptations, has given to each present species its own exact anatomy and histology.

Suppose the muscle becomes more muscle by dint of muscle-ing; the case is exactly the same as with the protoplasmic elements which struggle against toxins or microbes - the colloid organ is created by repeated colloid functions.

On the contrary, suppose that, in adult animals, each 
definite tissue has come to the term of its differential evolution. There is no longer any variation possible in the protoplasmic nature of each element, but only quantitative augmentations or diminutions without functional variation. Now, in an adult, the destructive reactions of the tissues counterbalance their constructive reactions, since the animal remains the same, like to itself ; and for each tissue which no longer varies we have to speak of assimilation pure and simple, and not of adaptive assimilation. The only question is-What are the conditions determining in the tissues now assimilation and now destruction?

The same element M, which can work only according to its nature $M$, is successively a part of different organsfor a man or a cat never does twice in succession the same thing. According to the successive functions into which the history of the vital activity of a man or a cat is decomposed, the element $M$ exercises its proper function M a great deal, or little or not at all. If it destroyed itself in accomplishing its function, it would be the frequent repetition of the function which tended to make it disappear - contrary to the law of habit. The smithing qualities of a blacksmith are developed in proportion as he works. On the contrary, an idle man's muscles are destroyed as muscle and loaded up with fatty reserves which, as we have seen, are products of destruction.

Consequently when, from the descriptive point of view, we are uncertain as to the peculiar function of any tissue in an organism, we have to study the conditions in which the tissue develops quantitatively while still remaining like to itself.

We are now able to express in all its generality the law of functional assimilation-the fundamental law of all biology. Relying on Claude Bernard, most physiologists 


\section{THE NATURE AND ORIGIN OF LIFE}

have preferred the law of functional destruction. This will have it that every tissue that works destroys itself, to assimilate afterwards in repose. It is clear that Claude Bernard laid down such a law à priori and without mature reflection; it renders incomprehensible the law of habit and the adaptation of the organ to the function. 


\section{CHAPTER XX}

\section{FUNCTIONAL ASSIMILATION A GENERAL BIOLOGICAL LAW}

LET us take a living body $\mathrm{A}$, formed of parts $a, b, c, \ldots$, each capable of multiplying on its own account, but incapable of variation in its own peculiar nature, because each has arrived at the term of its differential evolution. Such an example is, perhaps, to be found in certain histological elements of mammals. Any definite function in the body A has to define a mechanism composed of the parts $a, b, c$, but in such way that some of the parts $a$, some of the parts $b$, and some of the parts $c$, will be at work effectively in the mechanism, each according to its own peculiar nature, while, on the contrary, other parts $a, b, c$, will be in functional repose. The law of functional assimilation expresses the fact that the former are the seat of phenomena of assimilation, while the latter are the seat of phenomena of protoplasmic destruction.

If the living body $\mathrm{A}$ is a unicellular being, we cannot recognize the parts called $a, b, c, \ldots$, which compose it and which are capable of independent multiplication and destruction. Otherwise we should be able to apply to such unicellular beings, decomposed into tissues, the law as we have expressed it. Our ignorance of the independent parts $a, b, c, \ldots$, obliges us to lay down the law under a different form, as follows: When a sum total of circumstances $\mathrm{B}$ defines in the body $\mathrm{A}$ the function $(\mathrm{A} \times \mathrm{B})$, the 


\section{THE NATURE AND ORIGIN OF LIFE}

body $\mathrm{A}$ develops and multiplies as an organ of the function thus defined, so long as the sum total of conditions B which define the function $(\mathrm{A} \times \mathrm{B})$ remains the same. We have already had examples of this law in the increased virulence of victorious bacteria.

Among the histological elements of higher animals, along with those which have terminated their differential evolution (the constructive elements of the mechanism as a whole), there are others, such as phagocytes, which are capable of variation in the same way as the unicellular organisms of which we have just spoken. To the latter we have to apply the law of functional assimilation in the second form indicated, whether the function which they exercise is directly defined by a personal colloid enemy or indirectly through the intermediary of the mechanism as a whole constituted by the entire animal.

In the case where the animal observed can be decomposed into tissues capable of multiplying independently but without variation, the strict law of functional assimilation leads us to apply the law of assimilation pure and simple to the parts of the whole during the periods of functioning. In the fifth part of this book I shall try to show that the two methods of investigation employed in the third and fourth parts lead to concordant results, provided only we apply the language of the first method to elements capable of independent multiplication, but not of variation-elements into which we can always either effectively or theoretically decompose the mechanism of the more complex beings, to which the second method directly applies.

This agreement in the results obtained by the natural and the artificial methods of investigation has the very great advantage of bringing into agreement the two great schools of biologists - that of Lamarck and that of Darwin. 


\section{PART V}

Concordance of Results obtained by the Two Methods-Agreement of Darwin's System with that of Lamarck 


\section{CHAPTER XXI}

\section{NATURAL SELECTION}

There is one capital difference between the two methods as we have explained them.

In the first we consider assimilation pure and simple as an approximate law, on account of different variations due to any destructive actions whatsoever and superposing themselves in any manner whatsoever on the results of assimilation taken strictly.

In the second, on the contrary, we do not separate the assimilation from the variation; we verify a result as a whole, a synthesis comprising the whole activity of the living body under the influence of all the surrounding circumstances considered together. We also verify that results thus obtained are subject to the law of functional assimilation, the immediate result of which is the adaptation of organisms to the environment.

With the first method we consider individual A as multiplying in its own likeness and then undergoing superposed variations, caused by the influence of the conditions of the environment included in the term B. With the second method we know nothing about the organism A in itself; but the organ $\mathrm{A}$ of this organism, as defined by the function symbolically represented in the formula $(\mathrm{A} \times \mathrm{B})$, multiplies by functional assimilation as the organ so defined and conse- 
quently, from the start, must give a result adapted to the environment $\mathrm{B}$.

In order to employ the first method we had to keep to considerations of results of the whole expressed in the chemical language of the quantities of substances, without paying any attention to the existence of a colloid mechanism; and yet this always serves as the intermediary between external actions and internal chemical phenomena. On the contrary, in the second method we always consider the environment as acting solely on the mechanism and determining secondarily, by means of this mechanism, chemical reactions directed ipso facto in the sense of adaptation.

In the two cases we have studied the same facts. We ought, therefore, in spite of the divergence between the methods employed, verify some agreement in the results. We succeed in doing this, thanks to a process of reasoning due to Darwin and called natural selection.

Darwin limited his study of the living world to the verification of the variations accompanying the multiplication of living beings, without attending to the manner in which the variations are produced. Lamarck, on the contrary, connected variations with the law of habit, as we have done in the fourth part of this book. In this way, by trying to bring into agreement the results of our two methods of investigation, we at the same time establish the concordance of the two great schools of Evolution-that of Lamarck and that of Darwin.

Let us take the same example-anthrax bacteridium-a type which has been thoroughly studied and is easy to explain. Under condition No. 1 (see page 52), in a proper culture bouillon at $35^{\circ} \mathrm{C}$, the anthrax bacteridium A multiplies by strict assimilation in its own unvarying likeness. Under condition No. 2, in pure water to which phenic acid is 
added, bacteridium $\mathrm{A}$ is little by little destroyed through variations in its properties. In a phenicated bouillon there is superposition of these two phenomena, that is, multiplication and variation at the same time. And if you transfer the bacteridia which have varied into a bouillon realizing condition No. 1 for the species, they will multiply in their own likeness, indeed, but with the variations acquired during the period of destruction.

Now the general fact which serves as a basis to all Darwinian explanation is as follows: Subject a species to any conditions whatever allowing it to continue to live; it multiplies with variations which, as soon as the cause of trouble is suppressed, are preserved identically like to themselves until some new destructive reaction intervenes.

When bacteridia develop in a dead medium like culture bouillon, we have no strictly exact means of studying the variations which they undergo. To verify such variations we must use the only precise reagent at our dispositiona living animal organism - with reference to which we can define a really precise property of anthrax bacteridium ; this property we call the bacteridium's virulence for that animal.

Let us take any culture at all of bacteridia in which, without our knowing how, variations in different senses have been produced. Inject this culture into a sheep. This is a real Darwinian case, for the conditions in which the bacteridia varied in the previous dead media have no direct relation with the sheep's fitness to survive. Therefore, even if you agree with Lamarck that these variations were directly adaptive to the conditions in which they were produced, they were without relation to the interior medium of the sheep, in which different conditions are realized.

Here the sheep plays the part of a sieve. Those bacteridia which, after undergoing the variations, chance to be 


\section{THE NATURE AND ORIGIN OF LIFE}

virulent to the sheep, that is, fit to survive in the sheep, will develop in the interior medium of that animal. On the contrary, those which, after variation, chance not to be virulent to the sheep will die in the interior medium of the animal because, as the definition of non-virulence implies, they are not fitted to multiply in that interior medium. The virulent bacteridia develop alone and kill the sheep ; and if afterwards we search in the dead sheep's blood, we shall find only virulent bacteridia. The sheep, once more, plays the part of a sieve which lets pass only the virulent individuals fitted to prosper within it.

Here, indeed, is adaptation after the fact. Darwin gave the name of natural selection to this sieve function which keeps back all unfitted individuals and destroys them, and lets pass only the fit. Herbert Spencer, to the same phenomenon, gave the name of survival of the fittest-an equivalent of the other. It is clear that the example chosen is a case of pure Darwinism : it verifies, in fact, natural selection by a sum total of conditions differing from that in which the variations to be selected were produced. The variations among which selection is made are, therefore, really chance variations with relation to the sieve that chooses the individuals to survive.

But it is also a factitious example. Generally speaking, there are not in nature experimenters who take it on themselves to sift in a living sheep's body the bacteridia which have varied by chance in a culture bouillon or other dead media. We have been studying a case of artificial selection after the fact; but the case is none the less interesting since it clearly lays down the Darwinian doctrine at a point where the most convinced Lamarckian would be unable to deny that the variations were fortuitous in relation to the selectionapparatus used. 
Genuine natural selection is realized in the case where the conditions $\mathrm{B}$, in which the variations have been produced, are at the same time the instrument of selection. In conditions $B$, if we accept the Darwinian doctrine, the descendants $A_{1}, A_{2}, A_{3}, \ldots$, of a living body $A$ undergo variations which, no matter of what kind they be, are evidently due to the reactions produced by the medium $B$ and yet have no direct relation with fitness to survive in such conditions B. Among these descendants some chance to be unfitted to survive in conditions B and consequently die. Others, on the contrary, chance to be fit to survive in such conditions and multiply in their own likeness so long as the conditions are not changed.

Thus the result is the same as in the Lamarckian doctrine since the only individuals that remain alive are adapted to the conditions in which they live.

Anthrax bacteridium gives us an example of such genuine natural selection in a celebrated experimental case. In the experiment of Pasteur, Chamberland and Roux, already narrated (page 89) in Lamarckian language, a bacteridium had been deprived of virulence for sheep or, at least, had too little to flourish directly in a sheep, but was still capable of living in a new-born mouse and was able to kill it. It was passed on to an older mouse, and then to an adult, and so on, and its descendants in the long run were again able to kill sheep. Now, in Darwinian language, this experiment is told as follows :

The bacteridium, as it passes through the mice, new-born, older, adult, everywhere finds the same sieve - mouse - a sieve that is finer and more precise in proportion as it is an older mouse. The bacteridium multiplies under these conditions, undergoing variations without order and in every directionvariations which have no reason to be directed in the sense of 
increasing virulence rather than in that of decreasing virulence (this, of course, is the Darwinian thesis). But among the bacteridia thus multiplying all those which chance to have undergone variation in the sense of diminished virulence are stopped short by the sieve, mouse, which destroys them, whereas those which, equally by chance, have undergone variations in the sense of increased virulence pass through the sieve mouse and survive. And so virulence increases in proportion as generations of bacteridia pass through the mice, that is, on meeting the same sieve which keeps on realizing the same selection.

The result-increased virulence-is therefore the same as in the Lamarckian thesis for all surviving descendants of the original bacteridia. In the experiment which we have described there is nothing to make us choose one interpretation rather than the other, since in both we only verify the final result of things.

In the case of the bacteridia a very great number of individuals have been produced without our being able to tell the number of those which have died as a consequence of being ill-adapted. And so we have no answer to give to Darwinians when they assert, on the faith of their system of interpretation, that many bacteridia chance to be ill-fitted for the struggle and succumb in the course of our experiments.

But the case is not the same when, instead of the bacteridia, we make our observations on the sheep which is their host. Suppose, in fact, that, quite contrary to what has passed in the previous experiment, the inoculated sheep should resist the anthrax. It must have digested and assimilated anthrax bacteridia, which proves by definition that the bacteridia with which it was inoculated were not virulent in its case. But this sheep which has been cured of a 
first benign attack of anthrax can victoriously resist a second inoculation of bacteridia more virulent than the first. It issues from this second battle more hardened than before and, at last, it will be able to resist an inoculation of the most virulent bacteridia we can produce after they have successively conquered non-refractory sheep.

Here there is but a single sheep-the same sheep-which, attacked by bacteridia more and more virulent and triumphing over them successively, has become better able to resist even the most virulent bacteridia in existence. Here, then, must be an instance of personal adaptation for the struggle against bacteridia. We cannot look on the refractory sheep as one chosen out-after the fact-from the great number of sheep, of whom some, in the chances of variation, were found less fit to resist anthrax and so died under the repeated attack of the bacteridia, whereas this one which the chances of variation rendered fit to resist survived. Not only this way of looking at things is inadmissible in the case, since there is but a single sheep concerned in it, but we can see how improbable also it would be, even in the case of several sheep, because of the precise character of immunity with regard to anthrax. There are just as many special immunities as there are different microbian maladies; and we should have to admit that chance always gives certain sheep just those precise qualities.

We can get out of this difficulty, and keep to the Darwinian explanation, by considering the sheep, not as a single and indivisible individual, but as an aggregate of cells, each of which can be studied in the language we have used for the bacteridia. The struggle will then no longer be localized between sheep and microbe, but between the cells of the sheep and the unicellular microbes inhabiting it. In this case, if we suppose all the cells of the sheep to be, like the 
bacteridia, subjected to chance variation, those which chance renders fitter to struggle against the anthrax will triumph and be preserved, thanks to the sieve anthraxbacteridia, which, on the contrary, will destroy in proportion as they are produced all those elements of the sheep which chance renders unfitted for the struggle against the bacteridia. And so, if the sheep survives, as we have supposed, it will be made up of histological elements sifted out by the influence of the anthrax bacteridia. It will, therefore, be more fitted to resist a new inoculation of the terrible disease.

This strict application of the Darwinian method of interpreting the facts, fantastic as it may appear, sets us on the way of a more reasonable explanation. The moment we try to explain the individual adaptation of an isolated being to new conditions of existence, we no longer consider the being as a unit but as an agglomeration of smaller units capable of independent multiplication and destruction. Then, by applying to these smaller units the explanatory process which is the proper language of Natural Selection, we arrive at a conception of the adaptation of the whole individual.

To apply this process we decompose the sheep into cells ; in reality we have no reason to consider the cells as being the least units susceptible of independent multiplication and destruction. Facts, as we know them nowadays, concerning the possible adaptation of an isolated unicellular being lead us to think just the contrary - that the cell itself, if you wish to apply Darwinian interpretations, will have to be considered an agglomeration of yet smaller parts, to which the Darwinian language will be also applicable. But in that case, the anthrax bacteridium itself will have to be considered as a mechanism and consequently as capable of individual adaptations resulting from variations in its structure by means of these smaller units. 
Consequently, if we apply the Darwinian language to the independent units which go to make up the cell, we shall find that the cell behaves exactly as it should in Lamarck's theory ; that is, having been long subjected to certain determined conditions, under whose influęnce it continues to live, it will fatally end by being adapted to these conditions just so far as its nature allows. There will take place in it partial assimilations and destructions which, guided by Natural Selection, will end by transforming its mechanism into the mechanism needed.

But this result will be obtained only in case the living body continues to live. When we subject a living being to new conditions we do not know beforehand that it will not die of them. All we can say is that, if it does not die, it will accustom itself to them just so far as its mechanism allows-no one does more than he can.

Thus, by a subterfuge, we apply the Darwinian method, not directly to the living individuals, but to the smallest independent units which go to constitute such individuals; and thus we patch up an agreement between Darwin's theory and that of Lamarck-between selection of chance variations after the fact and direct adaptation. But it is only a subterfuge and with it we in reality adopt whole and entire Lamarck's idea-that the medium acts on the inmost tissues of a living being by means of the mechanism which that living being is.

A great American paleontologist, Cope, without going into these considerations, has acknowledged that there may be two methods of variation in the living being-cinetogenesis and physiogenesis.

The first-genesis of variations by movement-comprises those cases in which the influence of the surrounding medium first sets going the entire mechanism of the individual and 
only secondarily, by the intermediary of this mechanism, acts on the smallest independent units of the individual, that is, on its chemical constitution.

This is Lamarckian variation.

The second-direct genesis of variations-comprises those cases in which the influence of the surrounding medium is directly exercised on the smallest independent units without passing by the intermediary of the mechanism. Such variations may, therefore, be no matter what so far as the mechanism is concerned ; some may be favourable to it, others unfavourable, as chance may have it.

This is Darwinian variation, in which selection intervenes only after the fact-a secondary adaptation by the disappearance of unadapted individuals.

A rough example will bring out the difference between the two. Take a rat-trap with the spring set: if I loose the spring the trap works as a trap, manifests its mechanism; I have acted on the spring in a way corresponding to the cinetogenesis of Cope. If, on the contrary, without touching the mechanism, I apply a coat of paint to the different pieces of the apparatus, I treat it as I would no matter what object that is or is not a rat-trap; I act according to physiogenesis. The example is bad because the rat-trap is not alive ; once it has worked as a trap it is unfitted to begin over again of itself, whereas a living mechanism after working is still fit to work again. ${ }^{1}$ But we find such apparatus endowed with functional assimilation nowhere outside the living world.

To tell the truth, if we search for examples of physiogenesis

1 A rat-trap would be alive if, while exercising its normal function of loosing its spring, it should impress on its constituent substances a chemical activity whose result would be a tension of the spring tighter even than before. 
in nature, we have great difficulty in finding them. What Cope calls cases of physiogenesis are often instances of the direct action of the medium on the constituent cells of an animal instead of direct action on its mechanism as a whole. But the cell is a colloid mechanism and, if the cell receives an impression as a mechanism, the variation resulting from it in its chemical elements is a direct adaptation; it is cellular cinetogenesis, but it is cinetogenesis all the same.

The only instance of real physiogenetic action is that in which the agent determining the vital activity is a dissolved (and not colloid) chemical substance, able to influence directly the chemical elements which enter into the constitution of the protoplasm. Such, for example, is the case of an injection of morphine into a mammal ; and yet an isolated mammal can habituate itself to morphine, which gives the phenomena a Lamarckian look.

This result comes from the reversibility established between the chemical phenomena and the colloid states of the protoplasm - a reversibility which we have already shown from the relations of cause and effect existing between colloid equilibrium properly so called and the chemical equilibrium between the particles of the colloid and their solvent.

A few words will sum up these questions of equilibrium between mechanisms of different dimensions. They will give us a more scientific explanation of individual adaptation than that which is drawn from adaptation, after the fact of Natural Selection, to variations of the smallest independent units. 


\section{CHAPTER XXII}

\section{EQUILIBRIUM AND HABIT}

A MAN, or any higher animal, may be considered to be a mechanism in the third degree, a mechanism of mechanisms of mechanisms.

$\mathrm{He}$ is first of all a mechanism of the same scale as his body-an anatomical mechanism. A man has arms, legs, joints, muscles, sinews, etc.; they are the interworking parts of his anatomical mechanism. Between these different parts exist communications established by the nervous system, which may be compared to a network of electric conductors transmitting orders to set going or stop movement in an industrial machine. Here the anatomic mechanism already ceases to be entirely of the same scale as the man, for nervous transmission is not directly visible and belongs to the order of magnitude of colloid phenomena.

Excepting perhaps the bones which, in the execution of a great number of movements, seem to behave like rigid levers comparable to the rods of a steam-engine, all the other parts of the anatomical mechanism show their activity as parts of the whole only as a synthesis of smaller movements which are of colloid or protoplasmic order. When a muscle contracts, the muscular substance which constitutes it changes its colloid state; these two phenomena-contraction of the muscle and change in the colloid state of its protoplasm-are so closely united by relations of cause and 
effect that we are forced to recognize in them, strictly speaking, one and the same phenomenon studied in two different scales. But with regard to the man, the anatomical phenomenon alone has direct meaning; it constitutes the locomotor manifestation and brings the animal into direct relation with exterior objects of the same scale as man.

"I take my pen, I dip it in the inkstand, I write." Here are phenomena which take place in the scale of man and are capable of purely anatomical description, as here given, in human language.

An observer of the order of magnitude of colloid phenomena would describe the same phenomenon quite differently. He would never speak of pen or inkstand or hand or writing ; the ink alone would still be ink for him. Such an observer would describe the changes in the distribution of the particles which are of his own scale; and the act, so simple for us men, would be for him an incomprehensible story of astronomical chaos.

Yet it would be the same phenomenon, but observed in colloid mechanisms. Only, as its adaptation belongs to man's order of magnitude, this would be lost on a colloid observer too small to get acquainted with human synthesis.

On the contrary, in the case of the struggle of a toxin with a histological element where the phenomenon itself is of colloid order, the colloid observer would describe it in a very simple and clear way; but for us men the fabrication of antitoxic serums is simply a wonder. And yet, thanks especially to the genius of Willard Gibbs, we are now able to speak of phenomena that take place on a scale smaller than our own in the synthetic language of equilibrium. For that matter, we can work out syntheses on a smaller scale than our own more easily than on a larger scale. If there is one being formed of nebulae, just as we are formed 
of colloids, we are just as ill-fitted for the synthetic study of its history as our colloid observer was just now to study us on the human scale.

But for the observer of colloid scale there would still be phenomena so small that he could not describe them in language of his own scale-chemical phenomena which, for the colloid observer, would be what colloids are for us human observers. But in living substances, at least, chemical movements can be attached to colloidal movements (just as we have verified that colloidal movements are attached to anatomical movements) by such strict relations of cause and effect that chemical phenomenon and colloid phenomenon are the expression of but one and the same activity in two different scales.

Here, then, we have three distinct scales, in which the activity of an animal or a man can be considered-the anatomical scale, the colloid scale, the chemical scale. In these three scales we meet with different mechanisms, chemical, colloidal, anatomical ; and we know that relations of cause and effect exist among the activities of these mechanisms of different scale. At least, this is evident for colloidal and anatomical mechanisms; it is less certain for the chemical and the colloidal mechanisms. Perhaps certain movements of the colloid scale, certain modifications of the colloidal state of a protoplasm, may be produced without changing the chemical state of at least some of its chemical constituents. The influence of colloidal variations on chemical compounds is limited to those compounds which, along with the conditions of life, exist in their relations to the colloid state in conditions analogous to those of carbonate of lime above $960^{\circ}$ Centigrade in relation to temperature and pressure. Facts which we shall have to study prove that this is the case oftenest realized. 
The equilibrium of the animal mechanism may therefore be studied in three different scales, chemical, colloid, and anatomical. Clearly, then, we shall meet with three kinds of causes of activity-ruptures of equilibrium-to set the mechanism going. There will be causes of the anatomical order of magnitude, colloid causes and chemical causes.

The anatomical causes are all bodies existing in the surrounding world in our human scale; stones, trees, hindrances to our locomotion, wind, rain, and so on. To these causes we respond by anatomical movements, the adaptation of which to the preservation of life is what we call the instinct of self-preservation. These anatomical movements are naturally accompanied by colloidal and chemical movements, but it is in the anatomical scale that we recognize their utility. They are the anatomical functions, or functions of the mechanism as a whole.

Causes of the colloid order of magnitude are those which act directly on the colloid states of our protoplasms and have no direct relation with our anatomical mechanism. Such are sonorous vibrations, foods (savour), toxins, etc., and perhaps also electric phenomena.

In certain cases the activity resulting from such causes is confined to the colloid field. This takes place, for example, when a toxin or food substance is injected into the interior medium of an animal. The phenomena which follow the injection are colloidal and so is the useful result (fabrication of an antitoxic serum).

In other cases the activity due to a colloid cause influences the mechanism as a whole and determines anatomical movements. The savour of a food moves us to swallow it or to spit it out; a discordant note makes us gnash our teeth or even take to flight.

Causes of the chemical order of magnitude are those able 
to act directly on the chemistry of our constituent substances. Such are chemical solutions, light or heat radiations, etc. Even here we never know whether such causes do not also act directly on the colloid state of our protoplasms. A chemical solution possesses physical properties; for example electric properties, which may act on the colloid state; and the same is true of light or heat radiations. It is a delicate problem to find out which is the really specific action of a given reagent.

For example, an injection of morphine probably owes its particular activity to its chemical nature. It is probable that it acts directly on the chemistry of our protoplasm and, consequently, if a colloid activity results from it, this must take place secondarily - for the reason that the modifications induced in the chemical status of the constituent elements happen to be those which influence the colloid equilibrium. In point of fact, there is no anti-morphinic serum, as may be easily understood. Since the action of morphine is chemical, the habit which results from it must also be of chemical order. Accordingly, we shall have one of two things:

Either the resulting modification has no influence on the colloid mechanism (this would be a case of Cope's physiogenesis) and then it is only natural that the protoplasm habituated to morphine and experiencing no colloid modifications should not influence the colloid state of the serum in which it bathes;

Or else the resulting modification has a rebound in the colloid mechanism of the protoplasm ; and then it is possible for a corresponding modification to be transmitted to the serum by the protoplasm. But this would be a colloid modification and would have no reason to connect itself in any way with the chemical nature of the morphine ; and so it would not be antimorphinic. In the same way the bite of a viper makes 
us writhe with pain and cry aloud-phenomena of anatomical order without result so far as the viper's poison is concerned.

A struggle against any cause of action can be carried on effectively only by means of phenomena of the same scale of magnitude. We struggle against mechanical obstacles by anatomical movements, against colloidal enemies by protoplasmic modifications, against chemical agents by chemical reagents. It may be said that we can struggle against the viper's venom by flight, which is a locomotor movement; but this is not a defence against the venom itself, but against being inoculated with the venom-an anatomical phenomenon. In the same way we avoid asphyxia, a phenomenon chemical in appearance, by getting away from the place where the air is unbreathable.

That which, precisely, is wonderful in present-day animals is the instinct urging them to avoid by means of locomotion colloidal or chemical combats, in which they know they would risk being defeated. This question does not concern us here ; it has to be treated along with that of the origin of species.

To sum up, the animal ${ }^{1}$ being a mechanism in the third degree, may consequently be subjected to causes of action in three dimensions ; in other words, it is in equilibrium with three orders of phenomena which, in nature, may be independent of each other. By means of the connexions which unite these three mechanisms it thus sets up relations of cause and effect among phenomena which otherwise would exist isolated from each other; the animal is one centre of the world. By man's intermediary, the movements of the stars and intramolecular activities may be brought to work

1 I always take animals as examples ; but all that is said is equally true of plants, only with them the anatomical mechanism is generally of very slight importance. 
together in one and the same activity! Lamarck's genius made evident this fact of prime importance-the medium acts on animals through the intermediary of the animal's mechanism. We have just seen how this intermediary mechanism may be considered in three different scales. All the phenomena of biology are reduced to establishing equilibrium, on the one hand between living mechanisms and exterior phenomena of the same scale, on the other hand between the three orders of mechanisms which exist, united by bonds of cause and effect, in one and the same living animal.

In the living animal the phenomenon of equilibrium takes a particular form that is characteristic of life-habit. Habit with living beings, equilibrium pure and simple with not-living beings, are the two factors of what we call the universal harmony. 


\section{PART VI}

Third Point of View-Conservation of Energy 


\section{CHAPTER XXIII}

\section{THE STUDY OF LIFE AND THE CONSERVATION OF ENERGY-NEW PROOF THAT LIFE IS A PHE- NOMENON SUBJECT TO THE LAWS OF MECHANICS}

ONE of the most important conquests of man's science in the nineteenth century was the discovery of principles of equivalents summed up in the general law of the Conservation of Energy. Energy manifests itself in the world in a great number of forms, mechanical motion, heat, electricity, chemical change, etc. In a system of bodies forming one complete whole, that is, in a system which neither borrows from nor gives to its environment, there disappears a certain quantity of the energy known in one or other of the forms enumerated; but, in other possible forms, energy appears in corresponding quantities-so that the sum of all these new quantities multiplied, each on its own account, by a constant coefficient, reproduces integrally the quantity which has disappeared.

The energy lost in a waterfall is integrally found again in the heating of its water and of the stones of its bed and, in a way much easier to verify and measure, in the electric energy of a dynamo driven by it, or in the chemical energy of accumulators charged by the dynamo.

The old ideas about life dated from a time when principles of equivalents were unknown. They are naturally in 
contradiction with the new law of the Conservation of Energy. An incomplete observation of living bodies started up in our ancestors' brains a problem of perpetual movement - a problem, the vanity of which has been demonstrated by modern science. It consisted in finding some means of making an isolated system produce continuous mechanical work without expending any provision whatever of energy.

Nowadays the theorem, Nihil ex nihilo (nothing is made of nothing) is applied to biological facts just as much as to the facts of mechanics, physics and chemistry. Only we must take care not to consider the living being as forming, in the extent of its body taken alone, a complete system of energy.

Every instant, it is true, the individual body occupies a limited extent of space and is capable of circumscription as a whole; but we know that this body A has not within itself all the elements of its activity. The working of the animal as a whole at a given moment must, on the contrary, as we have already seen, be represented by the symbolic formula $(\mathrm{A} \times \mathrm{B})$-a formula in which the term $\mathrm{B}$ represents the sum total of the external conditions intervening to determine the working. It is only in this whole $(\mathrm{A} \times \mathrm{B})$ that we can verify the law of the Conservation of Energy.

Every instant the animal receives from without light, heat, and other radiations and chemical substances like oxygen, and gives out to its environment radiations (radiant heat) and chemical substances (water-vapour, carbonic acid). From this enumeration, as taking place only intermittently in the higher animals, we omit food exchanges which are the most important of all (absorption of food, excrementation). There are also sound vibrations, olfactory impressions, etc. No animal exists without its environment. 
The living being, having three different scales of activity, is a wonderful transformer of energy. In the example of the waterfall charging the accumulator, the dynamo is an intermediary between the mechanical energy of falling water and the chemical energy of the oxidized lead plates. In the same way, in the living being the colloid mechanism is an intermediary between chemical phenomena and mechanical phenomena.

When we compare, from this point of view of the Conservation of Energy, two successive states of a living machine, we have to take into account, not only what it receives from its environment, but also the provisions of mechanical, colloid and chemical energy accumulated in the individual at the two times chosen for the comparison. This is very complicated. When a blacksmith has worked his forge for two hours, it is not easy to measure the quantity of mechanical work he has furnished, the quantity of oxygen, of reserves and foods he has consumed, the quantity of muscle he has constructed by functional assimilation, the quantity of sweat, water-vapour, carbonic acid, etc., which he has lost. And yet all this would be necessary to strike the balance of his profits and losses.

The most difficult of all to measure-I would even say impossible-is the consumption of the reserves localized in the tissues and the fabrication of living substance, such as that of the muscles. By the scales we can only know the total weight of the individual ; but whether inside of him there has been a substitution of living substance for reserve substances we cannot ascertain. So we have to make experiments of very long duration to find out the food value of given substances-a practical side of studies of energy in biology.

Interesting as this problem is, we cannot dwell on it here. 
To prove how difficult it is to solve, we have only to point to the differences of opinion among scholars with regard to alcohol. Is alcohol a food? In other words, is it capable of being transformed within an animal's organism into some other form of energy, chemical as in reserves of living tissues, mechanical as in work? I believe it difficult to answer the question negatively, but it is quite as difficult to bolster up a positive answer with experiments beyond dispute. We can always ask whether the alcohol itself has been used as a food or whether its presence in the animal economy has not determined a partial consumption of reserves, over which. we can exercise no direct supervision.

Another difficulty, perhaps even greater, comes from our inability to measure colloid states and to compare them with each other. A variation in the colloid state of a nervous cell, when not accompanied by chemical phenomena, may without any modification in the weight of the individual change its energetic value. And, when accompanied by chemical phenomena, these will consist in exchanges between the cells and the interior medium-and such exchanges also are accompanied by no modification in the total weight.

To use a rough but striking comparison, variations in the colloid state of protoplasm are like the variations in the tension of a string-unaccompanied by change of weight.

An animal that appears inert, that is, executes no apparent anatomical movements, receives from without through its sense organs impressions of light, sound, smell and so on, so that we might think these inflows of energy from without were extinguished in it without producing any modification to verify the principle of equivalents. But the phenomena of centripetal nervous transmission, when 
not followed by a centrifugal outflow determining anatomical movements, spreads and is lost in the brain substance and modifies along its passage colloid states by tightening the springs. The animal is aware of these modifications of the colloid state produced in its nervous cells; they give it the memory of impressions received-another subject of study.

The brain is, therefore, an accumulator. This accumulator can be discharged in various ways, either in an external and mechanical form (anatomical movements), or in a purely intra-cerebral form by transforming itself into other colloid states or by determining chemical phenomena. In the same way, intra-cerebral chemical phenomena may be accompanied by colloid movements which do not issue forth from the brain. In this case the phenomena are known only by the being in whom they occur; they are translated for him by thoughts. Such thoughts may be followed by mechanical acts (anatomical movements), if the influence of transmission of colloid variations goes beyond the brain; then they become manifest externally. The outside observer, who has no means of knowing either the colloid or chemical phenomena taking place in the brain of the animal and ignorant of the real origin of such anatomical movements, may believe in their spontaneity, that is, may imagine that the animal does work with nothing.

These few considerations are enough to make us understand the origin of the error, still widespread outside of the scientific world, that animals can create movement, whereas they are only wonderful transformers, subject like all transformers to the principles of equivalents.

It is impossible to study directly the transformations produced in totalities so complex as man or mammals. Each of them comprises several trillion cells and these cells react 


\section{THE NATURE AND ORIGIN OF LIFE}

on each other, thus giving extreme complication to the resulting phenomena of the whole. It is more profitable to study the manifestations of life in beings reduced to a single cell-and even among these to choose the more easily analysed. 


\section{BOOK SECOND_-FACTS}

\section{PART VII}

Phenomena of Life compared with Phenomena of Not-Living Matter 


\section{CHAPTER XXIV}

\section{MORPHOLOGY OF THE CELL AND ITS MOVEMENTS}

ALL living beings are made up of cells. Some have but one cell ; these are simplest in point of structure, at least, there are very simple living beings among them. It is of these we have to speak in this Second Book.

The cell, as observed under the microscope, in spite of great diversity in its specific form, can be reduced to a constant type so far as its essential parts are concerned. Fig. 6 gives the diagram of this general type. The living protoplasm may be divided into three essential parts: the nucleus in the centre; around the nucleus the cytoplasm; around the cytoplasm the cell-membrane, which

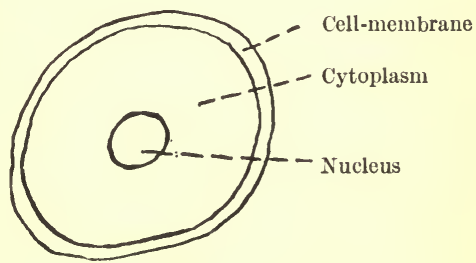

FIG. 6. should often be considered a particular phase of the cytoplasm's periphery in contact with the surrounding medium.

These are the living parts of the cell. Often, within the cytoplasm, there may be observed enclosed foreign matter, either the digestive vacuoles already mentioned or granules of reserve substances.

The cell-membrane is also often encrusted with not-living substances; sometimes even it is surrounded by a more or 
less thick layer of dead matter, often very rigid and highly mineralized. This protective wrapper formed of not-living substances is called the shell.

The cell-membrane as we have described it-a peripheral layer of the cytoplasm-is never wanting. Some authors speak of unicellular beings without the membrane; but they must be understood as meaning only this-the peripheral layer of the cytoplasm, though necessarily existing, does not take from its contact with the environment a very different appearance from that of the cytoplasm itself, not enough to deserve special mention when described.

Oftenest, when greatly magnified, the cytoplasm shows a rather complex structure. We distinguish in it certain more fluid parts filling the meshes of a network formed of the less fluid parts. What we have said of physiological division of labour helps us to understand that this must be so. The cytoplasm has a veritable histology of its own, but it is not easy to study.

Haeckel has described, under the name of monera, cells without a nucleus and reduced to a homogeneous cytoplasm. But progress in our methods of observation hardly seems to warrant faith in these monera.

In other species, on the contrary, there has been described a nucleus so voluminous that it may be considered as filling the whole cell; but here, too, there are remains of a cytoplasm.

On the side of dimensions there are tremendous differences in the various unicellular species. Certain rhizopods are large enough to be seen by the naked eye. There are micrococci so small that, even under the strongest lenses, they are seen only as geometrical points; and even then they have to be strongly coloured by suitable reagents.

For some years back there has even been current men- 
tion of invisible microbes. They are beings so small that, under the greatest magnifying power, they are imperceptible. And yet it has been possible to cultivate them in bouillons where their multiplication can be observed owing to their action on certain colloids or on certain living beings. They are known only by what they do. We are sure they are living because, by mixing a drop of the culture in which they are supposed to be alive with as much bouillon as we please, we can obtain just so much new culture having the same pathogenic or diastasic properties as the first. These very minute beings are, consequently, known only by the fundamental phenomenon of assimilation.

Certain authors think that these mysterious beings, endowed with assimilative powers, are not visible because they have no form or, in other words, because their living substance is soluble in the liquids in which they live. Had it been possible to verify such an assertion, it would have unsettled the whole general notion of cellular structure.

This, it seems, must be given up.

By using filters with pores sufficiently fine it is possible to stop on the way all invisible microbes known and to sterilize their culture. Until we know more, therefore, it must be taken as established that all living beings have a form, even when they are too small to be known directly by microscopic observation.

Nevertheless, the existence of species so extremely small takes all its value from the idea of cellular dimension. There are unicellular rhizopods as large with relation to the invisible microbes as the camel is with relation to a globule of its own blood.

One thing remains of importance in the idea of the cell, after putting aside Haeckel's monera and species too small for their structure to be known. It is the notion of small 
masses living isolated, endowed with assimilative power, and formed of colloid substances which their state enables them to distribute into two groups-nuclear substances in the centre and cytoplasmic substances in the periphery. In every case where their structure is well known, living cells must be considered as formed of two distinct colloid parts, just as an electric couple in the Voltaic pile contains zinc and copper.

Perhaps this comparison is nearer the truth than has hitherto been believed. Perhaps there are differences of electric order between the cytoplasmic and the nuclear protoplasms. The greater affinity of the nucleus for basic aniline colours might even be taken as a proof of these differences of electric order, if we can trust the interesting work of Jean Perrin on electrification by contact and on the nature of the part played by the substances called mordants in dyeing.

Such an approximation may be premature. But we find in nearly all vital phenomena a duality analogous to that of the two electricities, as shown in the sexual manifestations so general through the whole extent of biology.

Meanwhile, this comparison with the electric pile leads us to yet another remark on the dimension of cells. We can make the couples of the pile as large as we wish, with a very considerable surface; we can also make them very small, and we can leave them isolated or combine them. By a combination in quantity we obtain the equivalent of a single large-surface couple ; by combining their tension we obtain a result which no single couple, no matter how large, could produce. In the same way, we are acquainted with very large cells visible to the naked eye. We are acquainted with very small cells, invisible even under the microscope. We also know beings formed by agglomerations of cells and, 
because of this agglomeration, for the most part showing themselves capable of producing effects which no unicellular being ever produces whatever its volume.

The description of different cellular types belongs to treatises of zoology, botany or histology. We suppose the reader to be already acquainted with a certain number of types of living beings, to which, in his own mind, he can refer our abstract reasoning. 


\section{CHAPTER XXV}

\section{GENERAL PLAN OF MULTICELLULAR BEINGS}

Multicellular beings are constructed according to very different types varying from the chestnut-tree to man, not to speak of mushrooms and sea-urchins. Consequently it is difficult to give a morphological plan common to all such beings. In this chapter I have only to point out the consequences of a fact, namely, that we are now considering as entities by themselves, not cells, but agglomerations of cells.

Such an agglomeration has necessarily to be bounded by a contour-a surface separating it from the environment. Whatever is comprised within this contour we call the body of the multicellular being. But we have already seen that its contents are very heterogeneous; they comprise living cells (Fig. 7, P) and

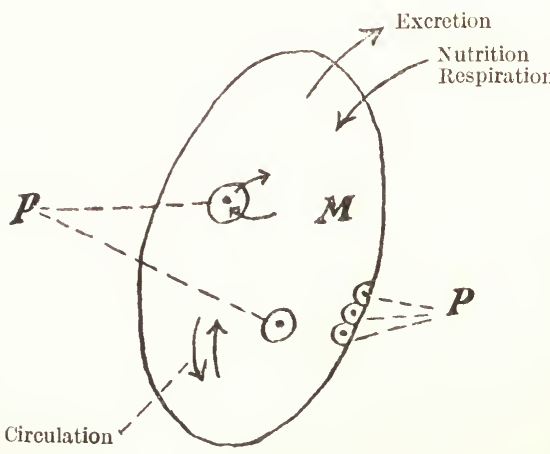

FIG. 7. intervals between living cells (Fig. 7, M).

To all that fills the total interval $\mathrm{M}$ of the living cell we give the $\mathrm{name}$ of interior medium.

Between this interior medium and the cellular bodies $\mathrm{P}$ are produced the exchanges 
which constitute the life of each of these cellular bodies. The collection of living cells draws from this interior medium all its food substances $Q$ and discharges into it all its excrementitial substances $R$. In general, the volume of the interior medium is very restricted with respect to the total volume of the living cells bathing in it. Consequently, the elementary life manifested in the cells could not be much prolonged unless the interior medium were renewed.

Now the result of all these concomitant cellular activities is shown by an activity of the whole multicellular beingan activity as a whole which should be called the life of the multicellular being and has precisely for its effect to renew the interior medium according to the needs of the cells. We may even make of this renewal of the interior medium an objective definition of the life of the multicellular being.

There is thus a double movement represented by the arrows in Fig. 7 :

1. A movement of ingress into the interior medium (nutrition, respiration), a movement of egress from the interior medium to the exterior (excretion), and a brewing movement of the entire interior medium (circulation). All this results from the co-ordination of concomitant activities of the living cells which constitute the multicellular being.

2. Exchange movements between the interior medium thus renewed and the various living cellular bodies P, bathing in this interior medium.

For example, in man oxygen enters into the blood through the lungs and is carried by the blood to all the anatomical elements, while the carbonic acid produced by these elements is brought back to the lungs and excreted. The diagram of Fig. 7 is as valid in the case of man as in that of the taenia or pear-tree. 
For higher animals like man, a remark has to be made with relation to the digestive tube which traverses the body of the animal from end to end (Fig. 8). The body of a man is a sack closed in every

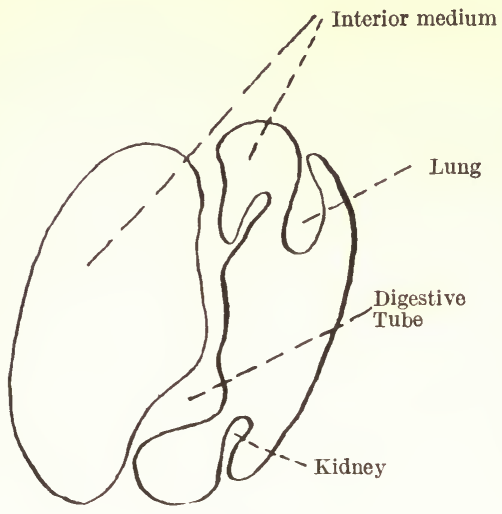

FIG. 8. part; the digestive tube traverses this sack, giving it, as we have said, the general plan of a lady's muff. But we must be careful to notice that the contents of the digestive tube are external to the body. When we eat we do not introduce food into our interior medium, but into an external cavity between which and the interior medium exchanges are set up (absorption, secretions, etc.), analogous to those which go on between the lungs and the blood.

In the interior medium of multicellular beings, particularly in the walls which separate the cells from each other, deposits are made of substances (excrementitial or reserves, according to the case), which are more resisting and rigid than protoplasm. These resisting substances solder themselves together and form a sort of network which is also resistant and encumbers the whole organism, serving as a support to the living parts. This is the skeleton. Its importance increases in proportion as the being grows old and, at each successive instant, it tends to fix the form of the being.

These remarks, however general, are sufficient to make the following chapters understood. 


\section{CHAPTER XXVI}

\section{SPONTANEOUS MOVEMENT}

A WIDESPREAD error consists in the idea that spontaneity of movement essentially characterizes life.

The error is easily understood because of the great part played in man's education by phenomena passing on his own scale. If we observe with no other help than what nature affords all that goes on round about us, it will seem evident that the mouse moves in conditions where a stone of the same dimension and situated in the same place would remain motionless. In other words, where the stone's immobility shows there is no cause of movement (wind, water-current, etc.), a mouse displaces itself spontaneously.

If we reasoned more closely, we should say:

Where the stone's immobility shows there is no displacing cause for the stone, the mouse's movement, on the contrary, should make us think-either the mouse is endowed with spontaneous mobility, or at the point where it is placed there is some cause of movement for the mouse.

To tell the truth, we are accustomed to seeing various objects, animate or inanimate, reacting according to their nature and in their own individual manner in presence of the same external causes. The breeze which flutters a piece of paper does not stir a pebble. Living beings are sensitive to causes which appear insufficient to determine variations in those not-living bodies which observation has 
rendered familiar to us; and, on the contrary, they seem to resist those external causes which are able to set moving inanimate objects. A trout goes up stream, while a cork goes down.

The name of irritability has been given to the property which living substances possess of moving under the influence of causes that seem incapable of stirring inanimate objects. Claude Bernard says that irritability is the property possessed by every anatomical element (that is, by the protoplasm which constitutes it) of being put in activity and of reacting in one certain way under the influence of external stimuli.

The same thing might be said of no matter what inanimate body. Everything is put in activity in a way proper to itself, by this or that external agent which would act differently on any other body. Claude Bernard's definition is therefore harmful, when, as is done in most treatises of physiology, it is pretended that irritability is the characteristic property of life. But it is useful if it means simply that the movements of living substances are not spontaneous, but are provoked by causes of motion. But even in this case it might be thought useless to create a new word; it would be enough to say that living bodies are inert just like others, that is, they are incapable of changing by themselves their state of repose or of movement. The word irritability is borrowed from the psychological history of man and gives the idea of some mysterious property opposed to inertia.

The same thing ought to be said of movement as of other manifestations of vital activity - in a given being A nothing takes place determined by A itself. Every biological phenomenon is the result of two factors-the living body A on the one hand and, on the other, the sum total B of exter- 
nal conditions capable of intervening in the determination of A's activity.

It is difficult enough to demonstrate this fact in an animal so complex as man, whose mechanism as a whole comprises several trillion cells co-ordinated by a highly perfected nervous system. In him, as we have seen, there may exist accumulated provisions of energy, either in the chemical form of reserves or in the physical form of colloid tensions. There are cases where it might be believed that he acted by himself because those portions of the medium with which he reacts are situated in his interior. But even then he still borrows from the exterior. We shall never see a man functioning without heat or oxygen.

The study of the agents of movement is easier in unicellular beings; among the simplest of them the movement provoked by an agent may have direct relation with the situation of that agent. On the contrary, with man the transformation in his brain of impressions received through the sense organs prevents our verifying any relation whatever between the direction of the movement and its causes.

As for that, the causes of movement in unicellular beings are so numerous and varied that, without the help of experimental methods, we should be tempted to believe in the spontaneity of their displacements. When, with our eye at a microscope, we observe a drop of an infusion of hay thick with protozoa and verify that two near neighbours among them are executing movements different from each other, our first idea is that these living corpuscles have the total cause of their activity within themselves.

The heterogeneousness of the drop of hay infusion is not apparent to the observer at the microscope. In the transparent liquid we do not remark the variations in oxygen, in salts of every kind, not to speak of local modifi- 
cations of the colloid state. All this passes unperceived, and yet a moment's reflection is enough to understand that the very fact of numerous living beings existing in this drop of hay infusion and there assimilating and excreting makes it impossible for us to consider identical any two points of the infusion, no matter how close to each other.

In the impossibility of analysing so heterogeneous a medium, the observer who is slothful or disinclined to reason prefers to conclude that the movement of infusoria is spontaneous.

Experimentally it is impossible to banish heterogeneousness from a medium wherein numerous cells are living; but we can arrange things so that one factor of movement, chosen beforehand and easy to direct, will act more energetically than all the others. We shall not thus obtain a movement produced exclusively by our agent, but the mechanical component which this agent introduces may manifest itself, verifiably and always the same, in all individuals of one and the same species so as to prove, with respect to the agent thus experimented on, the non-independence of the movements of the unicellular animals under observation.

\section{Tactisms and Tropisms}

The name of Tactism has been given to a property manifested by certain unicellular species-the property of being influenced in their movements by a given external agent. When the agent is light we have photo-tactism; when it is heat, thermo-tactism; when a chemical substance, chemicotactism, etc. Tactisms are, therefore, the elements into which we decompose the irritability peculiar to a protoplasmic species.

When the sensitive cellular elements are agglomerated in 
considerable masses as with plant cells, a great number of cell-tactisms taken together may result in giving an obvious direction to the growth of a branch; this is called tropism. Examples are heliotropism, the turning toward the sun of the sun-flower or heliotrope ; phototropism, as in a potatoplant sprouting in a cellar and turning toward a hole through which light passes ; geotropism, general in plants whose roots turn toward the centre of the earth, while the stalk mounts in an opposite direction. Tropism is a phenomenon of the same order as tactism. One type of well-made experiments in tactism will be enough for the reader to imagine others for himself.

From every point of view Pfeffer's experiments are a model of scientific exactness. Let us limit ourselves to the one made on the chemico-tactic influence of malic acid on the antherozoids of ferns. Fern antherozoids are small corpuscles extremely mobile in water, where they are observed. Probably their mobility is due to a great number of different causes and, by making one of these causes preponderant, we do not destroy the effects of the others. The movements are still very varied, but the particular development of their composition of forces is due to the action of the factor which has been introduced purposely.

Pfeffer introduced a dosed solution of malic acid into a small capillary tube, which he afterwards closed at one end. The open end of this tube he plunged into an infusion in which fern antherozoids were floating. It is clear that, under such conditions, the malic acid will be diffused through the infusion starting from the orifice $O$ of the capillary tube. The diffusion will naturally take place in such a manner that, at each successive instant, equal values of malic acid will be distributed through the liquid of the infusion in concentric spheres from the centre $O$ (Fig. 9, $\mathrm{S}_{1}, \mathrm{~S}_{2}, \mathrm{~S}_{3}$ ). 
In the liquid thus prepared let us consider a cell P. Between this cell and the medium physical and chemical exchanges will go on just as before. But there will now be something more-the action of the diffused malic acid. Now, along any straight line starting from the point $\mathrm{O}$ and penetrating the cell-body at the two points $a$ and $b$, it is plain that the point $a$ nearest to $\mathrm{O}$ will receive a stronger value of malic acid than the corresponding point $b$. The particu-

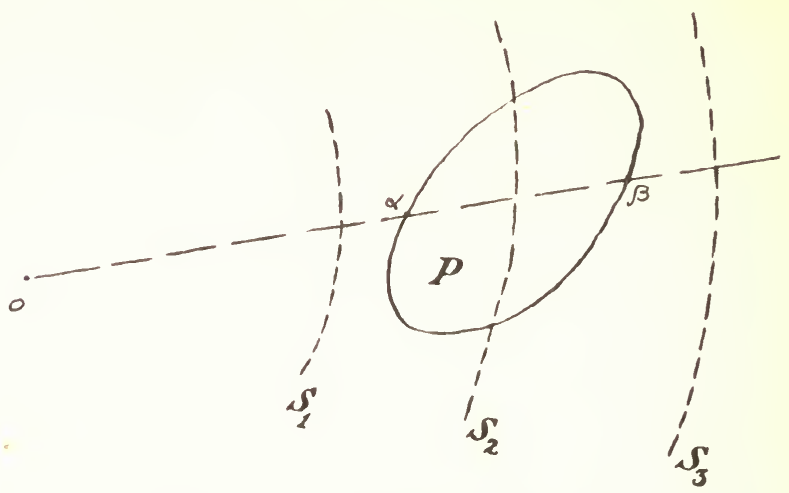

(Lines $S_{1}, S_{2}, S_{3}$, are arcs of circle with $O$ as centre.)

Fig. 9.

lar effect of malic acid on the antherozoids will, therefore, be stronger at $a$ than at $b$; and this will be true for all points $a$ in relation to corresponding points $b$ along all the straight lines starting from $\mathrm{O}$.

Without pushing the geometrical analysis of the phenomenon any further, it will be understood that these differences which are all in the same direction relatively to the point $\mathrm{O}$ form a component passing by that point $O$. In fact, in Pfeffer's experiment the antherozoids, while keeping up their very capricious movements in the liquid, ended-all of them 
-whenever the dose of malic acid was properly arranged, by being drawn into the capillary tube.

Such a capillary tube became a real trap for fern antherozoids.

This was an elegant way of showing the influence of malic acid on the movements of fern antherozoids. By repeating the same experiment with all the chemical products whose heterogeneous distribution through the liquid might influence the antherozoids' movements, and by analogous experiments which are easy and have been done with various physical agents (light and heat radiations and the like), the very complex movement of antherozoids can be decomposed into all its elements. In other words, the irritability peculiar to this cell species can be thus reduced to a sum of perfectly well defined tactisms.

After this nothing remains of the pretended spontaneity of movement in living bodies. An observer conversant with the results of all these experiments in tactisms knows that the movements he observes in living bodies through the microscope are due to the colloid and chemical reactions of the mobile beings and the medium.

Nevertheless, it is impossible to know every instant the heterogeneous conditions realized in the drop of water and, consequently, it will be impossible for the observer to foresee at any given moment the movements which the infusoria are going to perform. And so, if he pleases, he may keep to the old manner of describing vital phenomena and say that infusoria move as they please, for reasons that remain unknown. 


\section{CHAPTER XXVII}

\section{MORPHO-GENESIS IN LIVING AND NOT-LIVING MATTER}

Movement is one of the peculiarities to strike us first in living beings, although some of them are deprived of it and the seeming spontaneity of others is pure illusion. But form is also a most remarkable characteristic for recognizing living species. Generally speaking, we describe species solely by their form; and with a minute description of this quality we are certain to recognize, everywhere and always, the greater part of the living beings catalogued by zoologists and botanists.

In many cases, however, the form of a living being is kept by its corpse ; and we know that the corpse is not the same thing as the living being. It is always with the dead bodies of animals, cut up fine to be observed under the microscope, that we observe their histological structure. For this purpose we take the precaution of killing such beings in a very special way, by means of what are called fuxative reagents rendering the structural characters of the living being permanent in the dead body. This, of course, is very important from the descriptive point of view and is besides the only known process which reveals in detail the histological structure of living beings. But it is clear that such a method stands in the way of our eatching in the act the genesis of forms.

In the inanimate world we are also acquainted with characteristic forms of chemical species-the crystalline forms. 
Except in cases of isomerism, the measure of the diedral angles of the crystal enables us to know its composition. But even this supposes that the crystal has not been moulded hollow in a plastic substance and subsequently filled by a flow of glass or any other fusible substance. Geologists are acquainted with such mouldings in what are called pseudomorphoses sometimes found in nature.

But a crystal is essentially solid; the recent discovery of liquid crystals is really a play upon words like that which speaks of the life of dead substances. The crystal is solid, that is, under the conditions in which we observe it, ${ }^{1}$ it carries its form with it independently of external circumstances. For the environment in which we live we may say that the crystalline form of quartz is a property of quartz, even considered independently of all variations in the environment.

On the contrary, the form of living bodies, under the conditions in which they are observed by us who are also living, is the result of two factors-the living being itself and the medium in which it lives.

This is also true for crystals while they are forming; for, although certain characters remain constant in their geometrical form, there are others which vary according to the conditions of crystallization. On this account one quartz crystal has its prismatic part longer, another its pyramidal part surbased; there are individual characters in crystals of the same species on account of the conditions of their formation. But as soon as they have been formed and are solid, they remain what they are without being subject to the least transformation in any medium where they are observed by us.

${ }^{1}$ This restriction has to be made because, in certain conditions which have been verified experimentally, the most resistant crystals can be liquefied. 


\section{THE NATURE AND ORIGIN OF LIFE}

Living bodies unlike crystals are always, so long as they are living, under way of formation. At each instant of the life of a cell its form is the result of an equilibrium established between its content and the surrounding medium. Only when we have killed a cell by means of a fixative reagent (osmic acid, bichloride of mercury), does its form become definitive and transportable independently of external conditions like a crystal.

Here there is a fundamental difference between the form of living bodies and that of crystals. This difference evidently attaches to the fact that the colloid protoplasm is not solid like the crystal and is not capable of a form independent of the media through which it passes.

And yet, in many cases, living bodies seem to have as fixed a form as the hardest crystal. This comes from the existence of the skeleton.

Let us take protoplasm which for a long time is subjected to constant conditions and in which, consequently, morphogenic factors that also remain the same build up a constant form. Now suppose in the midst of such protoplasm a precipitation of solid substances (reserves, excrementitial substances) which agglomerate with each other to form a resisting cage that imprisons the whole colloid substance. The skeleton, thus built up by the living being itself, will be henceforth carried along with it through all the media which it traverses and will be a new morphogenic factor. Such a factor may even become so important that all others disappear before it and we no longer have an active protoplasm, creator of forms in the environing conditions, but a passive halfliquid clothing a solid skeleton as the waffle-paste covers the waffle-iron or, more simply, as water in a bottle takes the bottle's form. 


\section{CHAPTER XXVIII}

\section{THE MORPHO-BIOLOGICAL THEOREM}

Notwithstanding all that has been said, even in cases where the skeleton either does not exist or is not resistant, even in the case of a soft-bodied species, we recognize the species of a being, unicellular or multicellular, in spite of its displacement through the conditions of the medium.

Although soft-bodied beings are deformed during displacement, there still remains something common to all their forms, to their successive aspects. And on this account, so long as they are living, we can commonly recognize them.

There is one rare exception manifested in bacteria which drag out a precarious existence amid the unfavourable conditions of a culture medium left unrenewed for a long time. In such media deprived of their food substances and loaded with excrement, certain bacterial species take strange forms called involution-forms. A bacterium which in new bouillon would have the form of a straight little stick takes under these unfavourable conditions the form of a tear or spheroid. But if, betore death occurs, we sow such involution-forms in new bouillon again, we obtain a culture of normal bacteria. We can account for the origin of these involution-forms by remarking that, in the unfavourable conditions, the phenomena of our Condition No. 2 carry the day over those of Condition No. 1 (page 61). It is moribund and not living bacteria which we observe. 
Apart from this special case, through all changes of the medium, however considerable, provided they do not diminish the being's vitality, its form changes so little that we can always easily recognize it. But when the conditions are such that the being continues to live, we know that by assimilation pure and simple or by functional assimilation it keeps very nearly its colloid and chemical properties. Here we already have a glimpse of this remarkable result-in conditions of survival, where constructive phenomena triumph over destructive, even a soft-bodied being preserves its personal form with so little change that the eye can always easily recognize it.

On the other hand, we know that the form of a soft-bodied being depends not only on its bodily structure, but also on the mechanical conditions realized in its environment. A drop of not-living viscous substance carried around in whirls of running water takes the form of the whirlpools. On the contrary, a drop of living substance, so long as it continues to live through considerable variations of mechanical conditions, keeps its form with little modification although the form certainly depends on the mechanical conditions which are realized outside of it. The evident conclusion is that, while it lives, a living substance by the act of living creates in the environment around its semi-fluid body whirlpool movements which count for a great deal in the determination of its own form. Thus, just so long as it survives, the living being carries along with it, not only its own substance, but also a certain system of movements which it imposes on the medium in the immediate environment of its body. This is important enough to be verified experimentally.

First of all, we can easily understand the origin of these whirlpool movements. The living mass, plunged in the watery medium, occupies a limited volume in this medium. 
The life of the living mass - a sum of reactions passing between the living body and the medium-is manifested in every point of the protoplasmic substance, thanks to the penetration by osmosis of matters taken from the medium (food substances, the $Q$ substances of our equation of elementary life as manifested), and to the issuing forth, by diffusion, of the $\mathrm{R}$ or excrementitial substances. So there is a double current, ingoing and outflowing (Fig. 10), a current which is invisible under the microscope, but which is necessary for the constant re-establishing of the equilibrium of exchanges constantly destroyed by assimilative reaction. We shall see that the nucleus takes part in all these phenomena of exchanges; for that matter, the nucleus is limited within the cytoplasm just as the cyto-

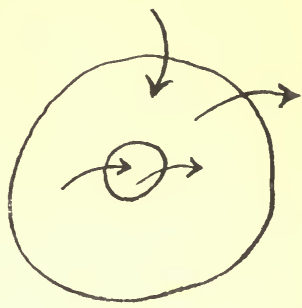

FIG. 10. plasm is limited in the medium, and by whirlpool mavement of the same order.

This exchange movement between nucleus, cytoplasm and medium gives rise to a state of incessant motion whose first result is to limit around the nucleus, which is itself limited, the possible volume of cytoplasm mass in equilibrium. This explains how growth, which is due to assimilation, is necessarily accompanied after a certain time by a fragmentation of the living mass (multiplication of bacteria, cellular division).

The system of movement determined by the exchanges depends on the colloid state of the protoplasm ; and this we can understand without being able to give an $\grave{a}$ priori demonstration, since we cannot define with precision the colloid state. It is enough that we should understand the origin of morphogenic movements ; experiments will teach us their re- 
sults. Current observation proves that as long as phenomena of assimilation have the better of destructive phenomena, that is, just so long as life persists, the protoplasmic form of the animal remains recognizable. Now we have the means of suppressing whenever we wish all phenomena of assimilation without changing any of the conditions of the medium; this means is the suppression of the nucleus. Here we have one of the most certain results of experiments in merotomy.

\section{Merotomy}

Experiments in merotomy consist in cutting the body of a living being in two or in several pieces; we then see if the pieces remain living and verify what is going on in such living pieces.

When the body under experiment is that of a unicellular being (Fig. 11) it is easy to observe that fragments deprived of nucleus immediately cease assimilating ; fragments containing the nucleus or a piece of nucleus, on the contrary, go on assimilating. The important result of the experiment is that the nucleated fragments which continue assimilating, after a short time, no matter how they may have been truncated, recover the normal form of their species. Cytoplasm and nucleus regain that form of equilibrium which they have in an ordinary individual that has not been subjected to merotomy.

On the contrary, fragments of cytoplasm deprived of nucleus, or fragments of nucleus deprived of cytoplasm, are susceptible only of destructive phenomena and are destroyed without resuming their specific form.

Except in the case of a rigid skeleton that hinders, these two results taken together prove that the specific form of equilibrium under normal conditions is a sign that phenomena of assimilation are going on in the being possessed of the 
form ; in other words, that the specific form of equilibrium is one of the consequences of assimilation in the protoplasmic being. I say consequences and not conditions, for the being truncated by merotomy is capable of assimilating if it contains a piece of nucleus, although it has not the normal specific form.

In other words again, the morphogenic function is a consequence of assimilation. At the same time that a living being creates protoplasm it creates protoplasm having a certain form. The morphogenic function is an aspect of assimilation and is not separable from it.

The moment there is assimilation there is determination of the specific form of equilibrium.

For that matter, we understand by "assimilation " two distinct, although inseparable things :

1. The fabrication of specific chemical substances;

2. The attribution to these specific chemical substances of the specific colloid state-the specific protoplasmic state.

This colloid state, of which as yet we are unable to give a detailed definition, has a primordial part in all biological phenomena. We already know it to be inseparable from chemical assimilation, of which it is not only the inevitable consequence, but also the necessary condition, so far as we can judge from the absence of phenomena of assimilation in all non-colloid bodies. But here the colloid state is attached to another phenomenon-the determination of the general form of the body-and yet here the connexion seems less close, for, while the specific form is a consequence of the colloid state of the protoplasm, it is not an indispensable condition for the maintenance of the colloid state. This is proved by the continuing of phenomena of protoplasmic assimilation in truncated beings.

The colloid or protoplasmic state appears, therefore, 
to direct constantly two equally noteworthy phenomena, to which it serves as a bond-chemical assimilation in the domain of molecular dimensions, and morphogenesis of the living body as a whole in the domain of dimensions of living beings. Here, again, we find the three mechanisms which we have spoken of as anatomical, colloid and chemical; but the colloid mechanism takes the leading place and dominates the two others.

This theorem, which experiment proves, of the existence of a bond between the specific form and the chemical constitution of living beings unites mor-

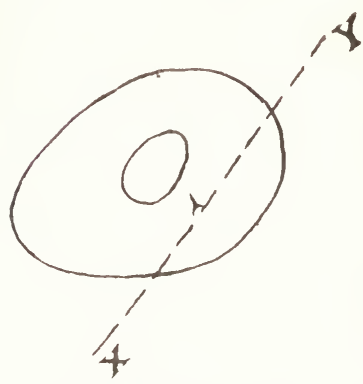

Fig. Il.-Digradi OF MEROTOMY. phology - the science of forms - with physiology, which is the science of activities. For this reason we call it the morpho-biological theorem.

Experiments in merotomy can also be made on multicellular beings as complicated as man and vertebrates. Any body can be cut in pieces and we can see if any of the pieces remain living and what happens to them. Of course, in such cases there is no longer question of nucleus and cytoplasm, but of some mechanism able to continue living, that is, to make sure of the renewal of the interior medium by proper exchanges with the environment. There will be as many different cases as there are experiments-the section of a small artery may involve the death of a man who has survived amputation of the leg. We shall have no general formula allowing us to foresee that the body will continue to live. But we can seek for a law of the phenomena that take place when the body does continue living. Now, at first sight, living beings seem to fall, without any apparent order 
under two categories quite opposite to each other from the point of view of the consequences of experiments in merotomy.

Under the first category fall beings which, having been truncated and yet surviving, regenerate their normal specific form. Such, for example, are the fresh-water hydra, starfish, crab, earth-worm, axolotl, lizard.

Under the second category fall those beings which, on the contrary, having been truncated and surviving remain truncated. Such are the sea-urchin, devil-fish, frog, carp, serpent, and mammals.

Does, then, the protoplasmic state with certain beings give direction to their form as a whole, whereas with others it has no influence on the form? This would seem all the more extraordinary as the animals divided up between the two groups belong to the same zoological classes or branches. The sea-urchin which remains truncated is an echinoderm like the star-fish which regenerates its arms ; the serpent is a reptile like the lizard, the frog an amphibian like the axolotl and triton.

On the other hand, if we make our experiments in merotomy, not on adult animals, but on the larva or very young embryo, we find in no matter what species that the regeneration of the truncated parts takes place up to a certain age, which is sometimes very advanced. The embryo of man himself, if the chances and changes in the matrix at the period when it is composed of two cells cut it in twain, gives a birth of twins, each perfect and complete.

Consequently, that which varies in the different species is not the absence or presence of the faculty of regeneration after being truncated, but only the age up to which the faculty persists. The morpho-biological theorem is general for living beings ; but, in the course of individual development, 


\section{THE NATURE AND ORIGIN OF LIFE}

sooner or later according to the special characters of the species, a new factor appears either veiling or even annulling its effects. A little reflection is sufficient to show us that this factor is the skeleton; and by skeleton, of course, is to be understood not only the bones, but also all resistant and not-living parts which encumber the being taken as a whole and become an important factor of its morphological equilibrium.

From the point of view of possible regeneration, we cannot yet estimate beforehand the value of the skeleton of a given species; we get our information after the fact from experiments in merotomy. In the present state of science, for example, we have no reason to foresee that the frog will remain truncated while the triton regenerates its leg. It is only after observation that we say-human substance clothing a one-armed skeleton will give a one-armed man. But this takes nothing from the value of the morpho-biological theorem when it explains individual development, for the parts fabricating the skeleton first fabricate themselves before they produce the skeleton that fixes their form.

Morphogenic Function and Importance of the Colloid State

Let us go back to unicellular beings. For these it has not been difficult to establish the morpho-biological theorem by experiments in merotomy. Regeneration is the rule for fragments provided with nucleus (with the exception of a species of ciliated infusoria-Paramaecia-which remain truncated just as a man remains one-armed). We conclude that, among the functions into which we can artificially decompose the total activity of protoplasmic being, there exists a morphogenic function inseparable from the rest.

This colloid function naturally does not manifest itself outside of the living body by any diastase diffused through 
the surrounding dead medium; it only extends, during assimilation, to the new parts gained by assimilation. Such a function does not seem transportable out of the living body like functions which are defined by resistance to a toxin. We may call it the function of the struggle for space. But, though not transportable, it is none the less well-defined and inherent in the colloid state of the protoplasm just like the other functions.

Moreover, an extremely important experiment shows that this function is really transportable into a dead body, namely, into the corpse of the living being itself when death has taken place under certain conditions.

When we kill a protozoon with ammonia we are utterly regardless of its colloid state, we dissolve it entirely (except the skeleton when this is resistant enough).

But in most cases its colloid state, on the contrary, survives the protozoon completely enough for us to recognize its corpse during a certain length of time, more or less long. Is the colloid state thus preserved the same as that of the living being from which the corpse has come, although no phenomenon of assimilation now takes place in the dead protoplasm? Evidently, the answer will depend on the manner in which the living being came to its death. Between the osmic acid which fixes the form and the ammonia which leaves not a trace behind, there are evidently intermediate cases of every kind-there are any number of ways of dying. The kind of death has therefore to be specified.

When a culture of microbes is killed by gentle heat, just sufficient to produce death, we do not know any too well what modification has been produced; but we can verify that the gentle heat preserves the form closely enough, even in the case of unicellular beings formed of nude, soft protoplasm. Yet transformations have been realized since life 
has disappeared-probably even there has been colloid transformation. But at least one colloid function has been preserved, the morphogenic function; I would almost say morphogenic diastase, since the function has been transported from the living body into the corpse.

Well! the importance of this morphogenic function is such that, if we inject into an animal the corpses of microbes, we vaccinate the animal against the living microbes themselves.

In other words, an animal digests, physically assimilates, corpses of microbes of the species A and develops in itself the organ productive of the diastase which digests these microbe corpses; and at the same time it becomes capable of digesting henceforward A microbes, even the living ones. In other words again, an A microbe which, in ordinary conditions, would easily live in a given animal and triumph over it in the struggle, cannot live when it meets with the diastase able to destroy the morphogenic part of its colloid state.

On the contrary, in most cases when we vaccinate an animal against one of the transportable diastases of the $\mathrm{A}$ microbe-against one of its toxins, as we say-the animal is not, on that account, vaccinated against the living microbe itself. This shows that the morphogenic function which is inseparable from the protoplasm of the A microbe is more indispensable to the life of the microbe than are the other functions which are transportable into the colloids of the environment.

We have thus taken a further step in the real and effective analysis of the vital activity of the $\mathrm{A}$ microbe by adding its corpse, which possesses its morphogenic or formative diastase, to those other diastases which transport into dead colloids its other partial activities.

Thus more and more the living being appears to us as a superposition of dead things. 
The practical importance of studying the morphogenic diastases of protoplasm is clear to every one willing to consider this fact; -in such morphogenic diastase, stripped as far as possible of other diastases and excrementitial substances, Koch first and Behring later have probably sought the cure of tuberculosis. By triturating the bacillus of tuberculosis, they have tried, as a means of curing man, to deprive it of its formative diastase. 


\section{CHAPTER XXIX}

\section{CONDUCTION PHENOMENA IN LIVING AND NOT-LIVING BODIES}

IN a living being of large dimensions like one of the higher animals or plants, the preservation of cell life in one point of the organism is attached to the renewal of the interior medium. So it cannot be considered independent of the localized cell-lives in distant points of the organism, for the reason that it is the whole of the cell activities taken together which results in the renewal of the interior medium useful to all. The brain cannot remain indifferent when the kidney is ailing nor the leaf when the root is cut.

The name of correlation is given to this community of interests which exists among the different parts of one and the same organism. It is established by the interior medium common to all the cells of the individual. If we introduce a poison at one point of the interior medium, diffusion and especially circulation will spread the poison everywhere; the local phenomenon of introducing the poison will be transformed into a general poisoning.

We can give a general formula of correlation from the fact that each cell has to be in equilibrium with the interior medium common to all. This equilibrium is at the same time of chemical and colloid order, since-and we must not forget it-the interior medium is composed solely of colloids. Now, with regard to serumtherapy and physical 
assimilation in general, we have seen how colloids bathing each other influence each other reciprocally. The circulation brews without stop the interior medium and gives it a certain homogeneousness in the individual as a whole. We ought, therefore, to consider all the cells of the living being-those of the eye as well as those of the toe-as being in equilibrium with one and the same colloid.

This already sets up a noteworthy unity through the being's whole extent; a modification of the colloid state cannot affect one cell without modifying the interior medium and having a consequent rebound on the sum total of the other cells. For this reason, in the individual, there is no local phenomenon.

By the interior medium, therefore, there is established a conduction of the local modifications toward the whole organism. Of course, this conduction will be weak in the case of a variation concerning at the start only a single cell, because of the slight dimension of the cell with relation to the whole body which in man, for example, is sixty trillion times greater. In the same way an electric oscillation, unless it be, very intense, is quickly spent when transmitted through the whole environment in wireless telegraphy. On the contrary, with a conducting wire, a weak oscillation may be transmitted very far without losing much of its intensity, because the quantity of substance set in motion is proportional to the length of the wire instead of being proportional to a power of that length, as in the case of wireless telegraphy.

This comparison with the telegraphy of Morse and of Hertz is apposite in the present case precisely because, in higher animals at least. along with conduction by the interior medium which recalls Hertzian telegraphy there is nerve conduction which recalls that of Morse. 
- Nerve conduction is nothing else than conduction by protoplasmic continuity. It manifests itself in any cell at all, but its effects are more curious when the cell is very long drawn out.

Consider (Fig. 12) a long cell A, B bathing in a different colloid from itself. If you produce at point A a rupture of the colloid equilibrium, this rupture of equilibrium will be transmitted from point to point on to $B$, where we can verify its effects. This does

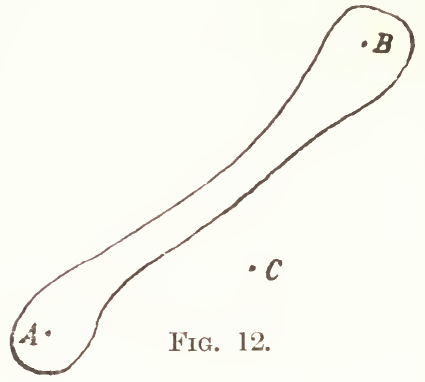
not prevent the rupture of equilibrium at $\mathrm{A}$ from having its rebound also in the colloid surrounding the cell and making itself felt at the external point $\mathrm{C}$; but observation shows that its influence at B is vastly more important from the continuity of homogeneous protoplasm. We verify the fact without trying to explain it, just as we verify in Morse telegraphy with naked wire that the effect transmitted by the conducting wire is much more considerable at the end of the wire than at any point of the surrounding air.

Experiments in merotomy make us understand the importance of protoplasmic continuity. After cutting a cell in two, the fragment deprived of nucleus still exists in the same drop of infusion as the nucleated fragment, but it does not experience in the infusion the beneficent influence of the nucleus. This makes itself felt by protoplasmic continuity only within the nucleated fragment, where assimilation consequently still goes on.

We can only verify the fact, but it does not surprise us. We are accustomed to recognize in physics the great import- 
ance of all heterogeneousness or discontinuity in conduction phenomena.

Even in the vegetable kingdom it is possible that phenomena of direct protoplasmic conduction take place through the whole extent of an individual by means of the little protoplasmic tractus so often described as perforating the separating membranes of two neighbouring cells. Still, on the one hand, the continuity established by the tractus is not sufficient to secure to the cytoplasm the benefit of the nucleary influence from neighbouring cells in experiments of merotomy; and, on the other, even when such continuity is real, it is from one cell to all neighbouring cells and so disperses through the individual as a whole the effect of the rupture of colloid equilibrium, which was produced at one point of the individual, just as in wireless telegraphy. ${ }^{1}$

When wireless telegraphy was discovered men cried out with admiration at the miracle. Later on, learning its grave inconveniences as a system of correspondence, they ended by observing that the real improvement to give it would be a wire, that is, they came back to telegraphy already known which, save in such exceptional cases as balloons and ships, is much handier for us. Just so, in the living animal we find, along with the wireless telegraphy which sets up correlation through the interior medium, telegraphing with a wire producing co-ordination by means of the nervous system.

\section{The Nervous System}

The rudiment of the nervous system consists in a cell

${ }^{1}$ A piece of protoplasm which has been deprived of nucleus in one cell by experimental section of the rest of the cell is fatally condemned to degenerate and die in spite of the protoplasmic relations which unite it with the neighbouring cell that still possesses its nucleus. 
long enough with relation to the individual to transmit at once, by protoplasmic continuity, a rupture of equilibrium produced at a point $\mathrm{A}$ to a remote point $\mathrm{B}$ in the individual.

We find elementary types of such an arrangement in the lower animals. One of the most characteristic examples is found in certain coleoptera described by Hertwig. Fig. 13 gives three samples of such protoplasmic continuity.
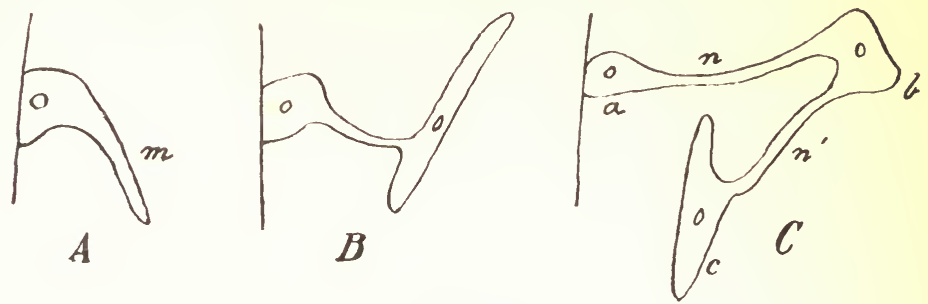

FIG. 13.

In the first type (A) an epithelial cell has but one prolongation $m$, which, on account of the conditions existing in the place where it penetrates, takes a contractile character (remember what we have already said of the physiological division of labour).

In the second type (B) the contractile prolongation has the look of a complete cell provided with a nucleus. It is a veritable muscle united to an epithelium by a nerve.

Finally, in the third type (C) a new cell $b$-an intermediate or nerve cell-appears in the continuous traject which unites the epithelial element $a$ to the muscular element $c$.

In the higher animals an entire network of protoplasmic elements lengthened out plays the conducting part between remote points of the same individual. Some of these elements are more than a metre in length; and we can understand, with respect to co-ordination, the extreme 
importance of these direct protoplasmic communications capable of making felt at once, in one point of the living being, a rupture of equilibrium produced at another distant point of the same individual. This transforms the individual as a whole into a unified mechanism wherein nothing that takes place in one part is indifferent to the other regions of the organism.

Neuron is the name given to a cellular element provided with a considerable protoplasmic prolongation which puts it in relation with remote cells of the body. This protoplasmic prolongation (cylindraxis) is of a very peculiar structure making it especially fit for the rapid transmission of ruptures of colloid equilibrium. According to the law of functional assimilation, it is natural that this cylindraxis, whose function it is to transmit, should acquire the character transmitter in the highest degree.

The cell which possesses this greatly lengthened cylindraxis has also other protoplasmic prolongations less differentiated; they form what are called the hairs of the neuron. In Fig. 14 we see the example of a neuron whose cylindraxis loses itself, by prolonged ramifications, in the protoplasmic substance of an epithelial element belonging to the external surface of the individual.

Let some phenomenon of the environment (of light or sound, colloidal or chemical) be able to make an impression on the protoplasm of the epithelial element and determine in it a rupture of the colloid equilibrium. This rupture of equilibrium will be transmitted from point to point by the cylindraxis to the body of the neuron, in which it will branch out in every direction according to the conditions of colloid equilibrium then existing in it.

The name of nervous influx is given to this protoplasmic transmission of a rupture of colloid equilibrium. It has 


\section{THE NATURE AND ORIGIN OF LIFE}

been possible to measure its velocity in certain cases, but there is no reason to believe it to be the same in all cases.

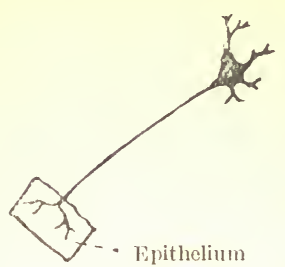

FIG. 14.

If the neuron's hairs represented in Fig. 14 were isolated from every protoplasmic mass, the phenomenon of transmission would be limited to the epithelial element and neuron taken together as a whole; the nervous influx would lose itself in the hairs of the neuron and its energy he transformed into chemical energy or simply into accumulated colloid energy, such as we studied in relation with the energy of life.

But things do not happen in this way.

The hairs of one neuron have a relation of contiguity or of continuity (there are different opinions on this subject and it is not easy to settle the question by methods of histological observation) with the hairs of one or more neighbouring cells (Fig. 15). Be it by real protoplasmic continuity or by contiguity sufficient to allow of a phenomenon of colloid influence, analogous to the phenomenon of electrostatic influence, what we observe is this-the influx is transmitted from neuron to neuron. Evidently the trans-

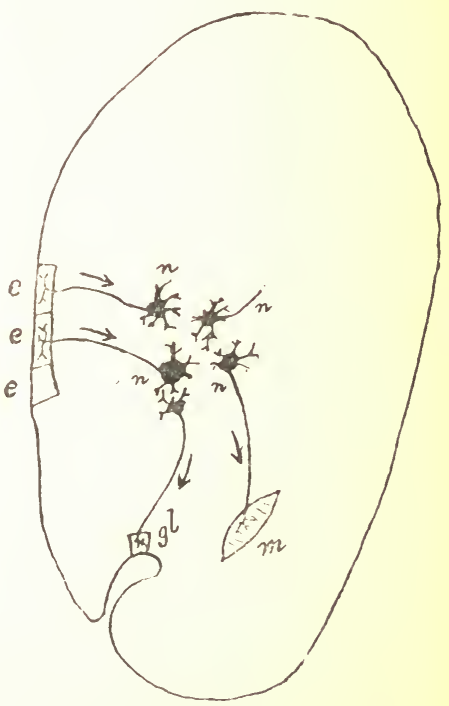

FIG. 15. mission is by the line of least resistance, as the particulars will show us later. 
Now suppose that, among the neurons into which a part of the influx may pass, one through its cylindraxis is in relation with a muscular element; the nervous influx will travel this way to the muscular element and determine in it a rupture of colloid equilibrium ; and this will manifest itself according to the nature of the muscular element, that is, by a modification in the state of contraction of the element.

Thus, by the intermediary of one or more neurons, a relation is set up between a surface impression received by an epithelial cell and a muscular movement which is a part of the functioning of the locomotor apparatus. This phenomenon has received the designation of "reflex"; it is an element of the individual's irritability, a part of the individual's manner of reacting against external stimuli according to its nature.

Consequences beyond measure flow from this disposition of things.

In the higher animals, indeed, it is no exaggeration to say that there is not one epithelial cell going to make up the outer wall of the animal's body (Fig. 15) which is not in relation with the extremity of the cylindraxis of a neuron. Now it is across these epithelial elements forming the body's surface that take place the exchanges between the being's interior medium and the environment-physical, colloid, chemical exchanges.

We see that the organism is well guarded. Nothing can enter in without making itself felt by means of the neurons in elements situated far from the entrance door. It is like some palace whose every opening is fitted with automatic alarms.

It remains for us to understand how all this mechanism is co-ordinated so that the muscular contraction, glandular 
secretions and the rest, which result from reflexes, have a precise utility in the struggle of the organism against the environment, that is, are adapted to avoid causes of destruction and to profit by favourable events. A cat draws back a burnt paw quickly (defensive reflex); the same cat, seeing and smelling a bowl of milk, approaches and laps it up (series of offensive reflexes). Such adaptation is studied by the science of the origin of species.

\section{Sense Organs and Sensorial Surfaces}

In an animal as complicated as the cat or man, the epithelial cells which line the surface of the body are not all alike. They are susceptible to a rupture of their colloid equilibrium from various causes. Causes of rupture from the outside are infinite in number and variety; it is probable that many have no action on the epithelial surfaces of animals. Among those whose activity is a matter of daily observation are light, sonorous vibrations, mechanical shock, heat, chemical or colloid substances.

With the lower animals it is probable that all the surface cells are more or less influenced in their equilibrium by these various agents. With the higher animals the division of labour which results, as we have seen, from functional assimilation, develops in certain cells a sensitiveness to certain particular agents. Thus light acts violently on the visual cells and produces modifications which are scarcely perceptible in the cells that clothe the hand or foot. In the same way, the savour of food (a chemical and colloid peculiarity) impresses itself on the cells of taste and has no action on the surface of the eye-and so on.

The name of sensorial surfaces is applied to those epithelial surfaces which are adapted to receiving impressions from this or that external agent. It is as if, in the palace just 
described, some of the gates were furnished with automatic alarms against certain robbers and gave no sound against others. Only, there is this to reassure the owner of the palace-in presence of robbers for whom the gates ring no alarm, they also do not open or, at least, do not open enough for the safety of the palace to be threatened.

In other words, every agent penetrating into the organism through a sensitive epithelial surface determines in the protoplasm of that surface a rupture of equilibrium which is the starting point of a reflex.

\section{Nerve Centres}

The bodies of the neurons $n$ (Fig. 15) are seldom isolated in the animal. Oftenest they are found associated in greater or less number in masses called ganglions, or nerve-centres. At these centres there arrive in great number the cylindraxes from various epithelial cells; these are called centripetal nerve filets because they bring the influx to a centre. From these centres there also issue a great number of cylindraxes, called centrifugal nerve filets, because they carry the influx starting from a centre either to a muscle or gland or to some other nerve centre.

We can easily understand that, when different parts of the body are in possession of their own special nerve centres, they are more or less independent of each other. In the general co-ordination they are related to each other only by the network of nerves which bring the centres into communication with each other.

In this book we have to occupy ourselves only with that which is general among all living beings. There is no need of lingering over the nervous system, since plants have but a rudiment of it (protoplasmic continuity). But in biology that which interests man the most is man himself, 


\section{THE NATURE AND ORIGIN OF LIFE}

and in man the nervous system is the most wonderful part of his mechanism; and so a few words have to be given to the consequences which flow from the existence of large nerve centres.

An influx arriving at the body of a neuron from a surface cell spreads through the neuron according to the actual distribution of the colloid states of its protoplasm. It is propagated, therefore, with variable intensity in the different prolongations of the neuron hairs. 'Thence it follows the points of least resistance and passes into the other neighbouring neurons to be distributed in their bodies in conformity with the actual distribution of the colloid states of their protoplasm-and so on. To be able to foresee the real distribution of a nerve influx in a centre composed of a great number of neurons, we should need an exact knowledge of the actual colloid state of the whole centre and of the relations of continuity or contiguity existing between the neighbouring neurons at the given moment. Now, all this varies every instant. There is nothing constant either in the relation of neighbouring neuron hairs or in the nature of the colloid equilibrium of each one of them. In fact, the previous influx modified everything where it passed and it will be the same for the following.

It is, therefore, impossible for an outside observer, when he determines in a higher animal a certain stimulus, to foresee how the animal will react to the stimulus. $\mathrm{He}$ knows that a nerve influx will be produced, but he cannot guess how the influx will be distributed in the centres and what motor apparatus it will reach and finally set in motion.

The outside observer's sole possibility of foreseeing is based on the law of habit. If he has remarked that, in given conditions, an animal often reacts in the same way to a certain stimulus, he is obliged to think that, by func- 
tional assimilation, the route followed by the influx several times in succession is more clearly traced in the nerve centres of the animal and that, under the same conditions, the same stimulus will determine the same activity of the animal as a whole.

A fisher with hook and line remarks that the flight of certain insects taken in a certain way along the river's surface makes the trout leap and snap at them; and so he will imitate as closely as possible with his artificial fly the motion of the natural one. If he succeeds, the trout will leap and swallow the hook. But for this it is necessary that the phenomenon should not be mixed up with other factors of action, such as sound vibrations-for example, when the fisher makes a noise-otherwise the observer will be unable to foresee the result of the experiment. $\mathrm{He}$ cannot tell beforehand whether the tendency to flight determined by the perception of sound will not prevail over the appetite determined by the bait.

\section{Trophic Rôle of Nerves}

In animals with highly differentiated tissues, we have seen that functional assimilation takes a particular form. A muscle which contracts and is already as much muscle as it can be, does not become more muscle; but it does grow as muscle by functional assimilation, when the contraction is really the function of muscle.

But we now see that what determines the muscle to contract or the gland to secrete, is the nerve influx arriving and determining a rupture of its colloid equilibrium. We are therefore brought to this interesting conclusion-at the same time that the nerve determines the functioning of a cellular element, its rôle with regard to the element is trophic or nutritive. 
A muscular element will in vain be irrigated by blood charged with food substances; it will not assimilate unless it is contracted under the influence of a nerve; and, not assimilating, it will be slowly destroyed.

In fact, when the nerve which ends in the muscles of a limb is cut, we see these muscles quickly atrophied although blood continues to reach them. We can sometimes obviate this partially by artificial contractions-electrostatic, for example-which bring the muscular elements into a state of artificial contraction.

Accordingly, the histological elements which are governed by nerves are, from the side of assimilation, incomplete elements. This explains the disappearance of useless parts in organisms and the development of those much used. From this point of view the history of the heart is interesting. In a normal adult the heart keeps a constant volume. During the periods of repose (diastole) it makes losses which exactly counterbalance its gains in the period of work (systole). But let any trouble in the general circulation prolong the systole with relation to the diastole and assimilation will become greater than destruction and we shall have a case of hypertrophy of the heart. 


\section{PART VIII}

Evolution in Living and Not-Living Matter 
a 


\section{CHAPTER XXX}

\section{HEREDITY IN THE WIDE SENSE AND INDIVIDUAL EVOLUTION}

WE have established a law-a living body behaves as it does at each separate instant under the influence of two factorsand these are easily distinguished in words. This law is equally true for not-living bodies. The word "functioning" applied to living beings is not ordinarily used in the history of inorganic bodies. We say rather "reaction" when there is question of a chemically-defined body transforming itself under the influence of new conditions, or "action" simply when no chemical transformation takes place. But, apart from this difference of the words we use, the verification of the law is general :-

A definite body $\mathrm{A}$, in a sum of circumstances $\mathrm{B}$, acts in a manner which at once depends on the structure of $\mathrm{A}$ and the nature of the factors of $\mathrm{B}$, in a manner which consequently admits of symbolic representation by the formula $(\mathrm{A} \times \mathrm{B})$. And the body which was called $\mathrm{A}$ before this action $(A \times B)$ becomes afterwards $A_{1}$ - a new body integrally defined by what $\mathrm{A}$ was and what the reaction $(\mathrm{A} \times \mathrm{B})$ has been.

One of the principal difficulties in generalizing this formula of evolution for not-living bodies is that, contrary to living bodies which continue living and preserve an individuality more or less easily defined, the not-living body is often destroyed in acting and always destroys itself in react- 


\section{THE NATURE AND ORIGIN OF LIFE}

ing chemically, so much so that it would be an illusion to seek the body $A_{1}$, into which the body $A$ has transformed itself. For example, if I pass a piece of coal A through circumstances B containing oxygen and a flame, I shall obtain carbonic acid, which may as rightfully be considered as coming from the oxygen as from the coal. The definition of individuality in not-living bodies is too fantastic for us to be able to follow such a body through successive transformations as we do for a living body, which remains recognizable, even though it undergoes profound modifications while remaining living.

In general, when there is question of not-living bodies alone, we ought to say: The body A existing under circumstances $\mathrm{B}$ results by action $(\mathrm{A} \times \mathrm{B})$ in a new state of things $\mathrm{C}$ defined by $\mathrm{A}, \mathrm{B}$, and $(\mathrm{A} \times \mathrm{B})$.

In this whole C, I may afterwards, as my fancy pleases, describe a body $\mathrm{A}$, and a sum total of circumstances B; but I might do this any number of ways. However, when we have solid bodies which undergo transformations slow enough for the continuity of their existence as solid bodies to remain obvious, we use the individualist language of living bodies and, doing so, commit the same abuse. That is, we preserve for the body we thus follow out the same name which it had at the beginning, in spite of the transformations it has undergone. For example, we treat a stick of chalk with phosphorus under suitable conditions; we obtain calcium phosphide, but the stick preserves its form as a parallelopiped, only it has turned chocolate colour. We say our chalk stick has been browned; it is a current manner of speaking, but it is erroneous. In the case of not-living bodies it is always preferable to speak of the sum total $\mathrm{C}$ of the results of a reaction; but, with living bodies, we cannot prevent ourselves from considering more par- 
ticularly what has become of the living body itself, while neglecting the environment or sum total B of the factors. We even always keep the same denomination for the living body after it has acted; and this is the origin of a great number of errors in reasoning, for we easily believe in the identity of two objects bearing the same designation.

The language we have so far used is exact: the living body A, executing under circumstances $\mathrm{B}$ the operation $(\mathrm{A} \times \mathrm{B})$ becomes $\mathrm{A}_{1}$. But so long as $\mathrm{A}$ remains living, $A_{1}$ sufficiently resembles $A$ (except in the case of metamorphoses which we have to study) for us to be struck by the resemblances which unite them rather than by the differences which separate them.

The term heredity in a wide sense is given to the sum total of characters which a body A carries with itself through the changes of its environment. When the animal is adult its heredity as thus defined does not seem to change much; the modifications which it undergoes, and which it can henceforth carry with it, are light enough to pass unperceived-and yet they are not, they cannot be null. Each instant the body A subjected to conditions B and executing the function $(\mathrm{A} \times \mathrm{B})$ becomes ipso facto a new body $\mathrm{A}_{1}$, determined by $\mathrm{A}$ and by $(\mathrm{A} \times \mathrm{B})$.

We give the name of individual evolution to the series of forms $A_{1}, A_{2}, A_{3}, \ldots A_{n}$, taken by the given being A from birth to death through the sums total of circumstances $\mathrm{B}_{1}, \mathrm{~B}_{2}, \mathrm{~B}_{3}, \mathrm{~B}_{n}$. These transformations, as we have seen, are such that the form $A_{2}$ is determined by the form $A_{1}$ and by the function $\left(A_{1} \times B_{1}\right)$, which may be expressed by the symbolic formulae-

$$
\begin{aligned}
& \mathrm{A}_{1}+\left(\mathrm{A}_{1} \times \mathrm{B}_{1}\right)=\mathrm{A}_{2} \\
& \mathrm{~A}_{2}+\left(\mathrm{A}_{2} \times \mathrm{B}_{2}\right)=\mathrm{A}_{3} \\
& \mathrm{~A}_{n-1}+\left(\mathrm{A}_{n-1} \times \mathrm{B}_{n-1}\right)=\mathrm{A}_{n}
\end{aligned}
$$


In current language these symbolic formulae signify that the being, considered at a given moment of its existence, comes from what it was at a previous moment and from all it has done in the interval.

The term education in a wide sense we apply to the series $\mathrm{B}_{1}, \mathrm{~B}_{2}, \mathrm{~B}_{3} \ldots \mathrm{B}_{n}$, representing the sums total of circumstances traversed by the living being from the point of its existence as $A_{1}$ on to $A_{n}$.

Taking into account the previous definitions, we can give a new expression to our symbolic formulae. The structure $A_{n}$ of a living being at a given moment of its existence-or, what comes to the same thing, its heredity at this given moment-is integrally determined when we know one of its previous heredities $A_{1}$ and the education $\left(\mathrm{B}_{1}, \mathrm{~B}_{2}, \mathrm{~B}_{3} \ldots \mathrm{B}_{n}\right.$ ) which separates the state $\mathrm{A}_{1}$ from the state $\mathrm{A}_{n}$.

We are accustomed to take as a starting point the initial heredity - the heredity of the egg - and to begin with the egg the series of terms B which constitute the education. In this case we say more briefly:

Every living being is a product of its heredity and its education.

This very simple formula has the great advantage of recalling at every moment that the life of a being does not dwell in the being itself, but is always the result of the struggle between two factors-the animal's body (heredity) and the sum total of surrounding circumstances (education).

\section{Assimilation and Morpho-Genesis}

Individual evolution as we have outlined its details may thus be considered from two points of view-that of chemical assimilation or fabrication of substances like those of 
the body which acts, and that of morphogenesis or fabrication of the successive forms of the individual. The morphobiological theorem, as we have established it, prevents these two points of view from being separable. There is indeed a relation of cause and effect between the fabrication of living substance and the corresponding construction of individual forms. Reciprocally, since the form of the individual at the point of its existence $A_{n}$ is an evident element in determining the functioning $\left(\mathrm{A}_{n}^{*} \times \mathrm{B}_{n}\right)$, the law of functional assimilation proves that the assimilation produced in the body $\mathrm{A}_{n}$ is not independent of the form of this body considered as a mechanism.

What has already been said of functional assimilation in cases where the division of physiological labour produces cellular differentiation dispenses us from further developing this subject.

The really important question relative to the individual evolution of living beings is to know how far, when the heredity of the being is known, we can foresee what its evolution is to be. Indeed, that which determines $A_{n}$ is not only $A_{1}$, but also the whole educative series, $B_{1}, B_{2}$, $B_{3} \ldots B_{n-1}$. Now, while we are? acquainted with $A_{1}$, we do not know a single term of the series B. Strictly speaking, therefore, we are obliged to think that it will be impossible for us to foresee anything at all relatively to $A_{n}$. Sera-t-il Dieu, table ou cuvette?

We do not know. The larva which we see may be eaten by a bird, crushed by a stone, transformed into a mummy in formic aldehyde. There are any number of "Perhapses," among which we are unable to choose beforehand.

But not all these cases concern the biologist. The problem of individual evolution does not consist in foreseeing what a living being will become under any and every con- 
dition, but rather in conditions such that the being continues to live. This singularly limits future possibilities. In fact, observation proves that the possible variations in a living being cannot go beyond certain bounds under pain of death. A young fox will become an adult fox and certainly not a herring or a horse. But this has nothing surprising in connexion with what we already know. A fox that continues to live can do nothing but "fox" and, while foxing, it builds up a fox by functional assimilation.

In other words, the conservation of life or renewal of the interior medium can be carried on only by the play of the animal's pre-existing organs, by the functioning of the tools which constitute its body. These tools, while functioning, develop themselves by functional assimilation. Under certain circumstances, one of them may develop itself more than another, which introduces into the economy of the individual quantitative variations. But even such quantitative variations are limited by the general co-ordination ; the hypertrophy of one organ and the atrophy of another cannot pass certain limits without involving the animal's death.

This is why we have the right to say that the heredity of the egg determines in a certain measure the future of the being which comes from it. We cannot foresee if the being will live or die ; but we can assert that, if it lives, its variations will be comprised within certain limits. In particular, we can assert that the being will belong by its morphological characters to the species which furnished the egg from which it issues.

In proportion as the being grows old, at least in species with a persistent skeleton like the human species, the variations allowed to it become more and more restricted. The skeleton which fixes all the parts of the body makes of it 
more and more a very exact and very exacting mechanism. This does not prevent an old man, for example, from becoming one-armed.

Moreover, we should remark that, even in the first periods of life, the conditions under which a young embryo develops itself are little variable. Without speaking of mammals or birds, in which the conditions of development are wonderfully constant, what variations can we observe in the sea water where an urchin's or herring's egg is growing ? There are, at most, a few variations in temperature or saltness ; and besides, when these variations pass certain limits, the embryo dies.

Thus it is generally said, with a degree of exactness which previous considerations allow us to estimate, that the animal is determined in the egg. We know beforehand that the animal which issues from the egg will resemble the animal which gave the egg; and so the current meaning of the word heredity is not different from that which we have given by strict definition.

In general, the term heredity is applied to the fact that children resemble their parents (leave aside for the present the difficulties arising from sexual reproduction, which causes the child to issue from two parents). Now this resemblance-heredity results from the structure-heredity of the egg, since this structure-heredity of the egg determines within certain limits the form of the child. But we must at once take notice that structure-heredity is simply a definition. What we call the heredity of the egg is its physicochemical structure, whereas resemblance-heredity is a biological theorem which daily observation demonstrates, namely, that the structure-heredity of the egg furnished by a being determines in the child of that being a morphological constitution resembling that of the parent, provided death 
does not step in. Now, the egg is generally very small and of a structure relatively simple in appearance (which only means that with our optical processes we can see nothing in it). The fact that so small an egg reproduces an animal endowed with so wonderful a constitution has always been considered one of the most marvellous phenomena of nature.

\section{The Structure of the Egg's Heredity}

The synthetic language which we have been using dispenses us from a knowledge of the structure of the egg's heredity or, at least, consoles us for not knowing it in the present state of science. The series of our symbolic formulae :

$$
\begin{aligned}
& \mathrm{A}_{1}+\left(\mathrm{A}_{1} \times \mathrm{B}_{1}\right)=\mathrm{A}_{2} \\
& \mathrm{~A}_{2}+\left(\mathrm{A}_{2} \times \mathrm{B}_{2}\right)=\mathrm{A}_{3} \\
& \mathrm{~A}_{n-1}+\left(\mathrm{A}_{n-1} \times \mathrm{B}_{n-1}\right)=\mathrm{A}_{n}
\end{aligned}
$$

decomposes the entire individual evolution of an individual into a series of successive functional assimilations, each of which separately is relatively simple. With the very wide definition already given of functioning, it is not difficult to use this language of functional assimilation as well for the chicken still imprisoned within the egg, as for the chicken that walks and eats. The functions are different, but they are always defined by the synthetic formula $(\mathrm{A} \times \mathrm{B})$ where $B$ represents all the external conditions of any importance in the determining of individual functioning. Accordingly, if we wish to explain how the chicken came from the egg, we have to go back from the chicken to the egg by decomposing its whole existence into the series of its successive functionings. Each period of the evolution will be explained by the period immediately preceding and the functioning that separates these two periods. This intermediate functioning depends on the factor $\mathrm{B}$, which is not comprised 
within the chicken. But, the moment the chicken continued to live, such functioning must have comprised, along with variable and fantastic parts the renewal of the interior medium fatally developing by functional assimilation certain parts of a chicken; and these, precisely, are the essential parts of its organism. Thus, through all the functionings that support life, the chicken-character is preserved.

This chicken-character already existed in the egg, without our being able to define it otherwise. This gallinity ("gallina," Latin for chicken) in the egg's heredity determines under pain of death development in the direction chicken. When we study under the microscope the structure of a hen's egg, we cannot distinguish gallinity in it. We define the gallinity either by the origin of the egg, or by the result of its development, when it exists in the conditions required for not dying.

This is exactly the same case as what we related of diastases. We can define them only by their origin, or their results ; and for the most part a complete definition would oblige us to know at once the origin and result. Now an egg is much more complicated than a diastase; in particular, it is constituted by a great number of superposed diastases which can sometimes be experimentally separated from each other. For the present, therefore, we do not pretend to be able to recognize the species of an egg otherwise than by its mother or its product.

Nevertheless, many naturalists long since proposed to explain by some hypothetic structure of the egg, the marvellous fact that the egg almost completely determines the evolution of the living animal that comes from it. They had not made the reasonings which we have made; they had not decomposed individual evolution into a series of 
functional assimilations, all comprised in the same general scheme.

The first and simplest opinion is this : there exists in the egg some very minute little being, similar to the adult and having only to grow. Observation has shown this hypothesis to be inexact; it left untouched the difficulty of successive functional assimilations, replacing them only by simple assimilations at each point of the persistent organism-something which excluded all possibility of variation. And yet it has been generally found so attractive that, when the microscope proved its falsity, scholars like Darwin and Weismann tried to revive it under an equivalent form-that of representative particles.

I have already (Chap. VI) argued against this artificial decomposition of an adult individual into characters chosen at random, such as would have to be represented in the egg by the invisible particles. Evidently the chicken is determined in the egg, since every time the egg goes right it brings forth a chicken. But the question is how it is determined; in other words, what are the chicken characters which exist in the egg, just as they exist in the chicken and in the adult hen? Every à priori hypothesis in such a matter can be only sterile. It is far better to use general expressions, so as not to compromise the future, and in order to narrate facts with as few implicit hypotheses as possible.

I propose the name of hereditary patrimony for that something which is common to the egg and the successive stages of the animal which issues from it. This hereditary patrimony is but a handy expression with a view to suppressing all such odd specific appellations as "gallinity," "herringhood," etc. It may be susceptible of decomposition into distinct elements of multiplication and destruction so inde- 
pendent that, in the course of the individual life, certain quantitative variations of this hereditary patrimony become possible under the influence of functional assimilation. These distinct elements are perhaps diastases, perhaps chemically-defined substances, perhaps both at the same time. When we wish to reason without compromising the future, we had best represent them by letters without pretending to know anything more about them.

We represent by our symbols $A_{1}, A_{2}, \ldots A_{n}$, the successive forms of an individual under development. If the development takes place under conditions favourable to the species, in conditions in which the species has lived for long generations and to which it is habituated, we may consider that the hereditary patrimony $a$ of the egg is transmitted integrally to the individuals $A_{1}, A_{2}, \ldots A_{n}$. But it is more likely, and more of a generalization, to suppose that the hereditary patrimony $a$ of the egg undergoes in the course of successive functional assimilations quantitative variations. Accordingly we say that, to the successive forms $A_{1}, A_{2}, A_{3} \ldots A_{n}$, there correspond hereditary patrimonies $a_{1}, a_{2} \ldots a_{n}$. In this way we shall not separate the question of heredity in the strict and rigorous sense from the far vaster and more general question of the heredity of acquired characters. Heredity without any modification of the hereditary patrimony transmitted from parent to child is only a particular and restricted case of the really general heredity of acquired characters. 


\section{CHAPTER XXXI}

\section{HEREDITY OF ACQUIRED CHARACTERS}

In the course of successive functional assimilations the hereditary patrimony of the egg is transmitted to all new parts which are added to the body of the growing individual. This hereditary patrimony is accompanied, in the various parts of the individual, by local or topographic charactersspecial colloid characters which make of this protoplasm a muscle and of that a neuron and of yet another an epithelium.

The child resembles the parent in virtue of the morphobiological theorem. If, therefore, there should exist at any point of the parent an element capable of living by itself, capable of withdrawing itself without dying from the general co-ordination-a reproductive element, in a word-there would be found an analogous element in a corresponding point of the child. (I again neglect the difficulty arising from sexual reproduction, which is to be studied in a chapter by itself.)

This reproductive element which appears in the child will have its hereditary patrimony from the egg which gave birth to the child through the successive variations determined by functional assimilation. The successive variations are always limited by the needs of conservation of life; and so we can foresee that the hereditary patrimony of the child-egg will differ very little from that of the parent-egg. We have even seen that when the 
animal lives under conditions to which its species has long been habituated," the "variations of the hereditary patrimony may be" considered null. Without appreciable error we may admit that the hereditary patrimony of the child-egg is identical with that of the parent-egg.

But when important modifications are produced in the life conditions of the species, some new adaptation will be necessary under pain of death. The animal, therefore, if it does not die, will vary by functional assimilation, that is, certain of its constituent parts will be developed more than others and these, on the contrary, will grow less. These variations in the general structure of a being are commonly called acquired characters.

In reality, in strictness of speech, we ought to say that every character is an acquired character, for, no matter how small the variations in the educative factors B may be, they are never null. We must not forget our symbolic formula-

$$
A_{n-1}+\left(A_{n-1} \times \mathrm{B}_{n-1}\right)=A_{n} .
$$

But, under conditions considered normal for a species because of prolonged habit, the variations may be neglected, since the children are always, at any determined age, very nearly what their parents were at the same age. In other words, the series of the factors $\mathrm{B}_{1}, \mathrm{~B}_{2}, \ldots \mathrm{B}_{n}$, is very nearly the same for the children as it was for the parents.

In cases where new factors $B^{1}$ are introduced into the education of the children, these, if they do not die, will adapt themselves by functional assimilation; and by such adaptation they become different from their parents.

The problem of the heredity of acquired characters is the following :

1 These B factors may be anything at all, sickness, mutilation, passage from land life to aquatic life, etc. Our reasoning is general. 
1. When a being, subjected to a variation under the influence of a factor $\mathrm{B}$, to which its species is unaccustomed, acquires thereby a character $\mathrm{C}$, is this character represented in the hereditary patrimony of the being?

2. Is the modification introduced into the hereditary patrimony such that the acquired character $\mathrm{C}$ will be inevitably reproduced in the child of the being thus modified, even when the factor $B$ which determined the father's variation at a certain age is not found at the same age in the education of the son? This question has commonly been understood in a quite different manner from that suggested by all our previous considerations. We, in fact, know that we ought not to consider the character $\mathrm{C}$ as a local modification analogous to what is produced by a stain on a statue. The living organism is a whole in equilibrium, and every rupture of the equilibrium realized at one point of the body is transmitted to the whole individual, either by the interior medium or by protoplasmic continuity. We have, therefore, a necessary answer to the first of these questions : ${ }^{1}$

The variation produced by the factor B most certainly had its rebound in the hereditary patrimony.

It is a more delicate matter to answer the second question.

Here the question is whether the variation, which inevitably reaches the hereditary patrimony by influencing in the patrimony the body's general state of equilibrium, remains fixed in the hereditary patrimony even after the original rebound is over. A spring keeps its tension as long as you press on it; but loses it when you let go.

1 The Neo-Darwinians, having adopted the static system of Weismann, naturally deny all possibility of transmission of acquired characters. 
For many very temporary modifications it is probable that the organism behaves like a spring. A man bent beneath the weight of a burden stands up when he has laid it down and takes his normal form; but if every day, during several hours, he remains bent, he will finally take the bend. The general equilibrium of the body certainly feels this; but we wish to know whether the egg, which shares in this modified equilibrium, is strongly enough impressed to carry with it, outside of the body which gave the impression, the corresponding modification; or whether it, too, will not relax its tension like a spring and cease being subject to the cause of variation.

So the problem is two-fold;

1. Has the cause of action B acted long enough for the body of the animal to keep the trace of it ?

2. If the animal preserves the trace, has the egg remained long enough in relation of equilibrium with the animal for it to keep the impression when it issues from the animal ?

Evidently, only in this latter case can the acquired character become hereditary. There is always the dovetailing of our mechanisms : the anatomic mechanism of the animal is moved by the conditions of the environment; it influences secondarily the equilibrium of the animal's colloid mechanism; and this, thirdly, may influence in its turn the chemical mechanism. Then the character will be very profoundly acquired, and, in such a case, the reversibility of chemico-physical phenomena on one side and the morpho-biological theorem on the other will enable us to make the following assertion:

"The modification transmitted to the hereditary patrimony of the egg will manifest itself in the next generation by a descriptive character of the same order as that which had been directly produced in the parent. Thus, in terms 
of equilibrium, this mysterious question of the heredity of acquired characters takes an extremely simple aspect.

Observation in general proves that for a character to be fixed definitively in the heredity of a race it has to be acquired successively during a very great number of generations.

\section{Rudimentary Organs}

When a character has been acquired by a race during a great number of generations under the influence of a factor $\mathrm{B}$, it is transmitted along the whole line of descent. There it is preserved and there it develops so much the more as the factor $\mathrm{B}$ persists the longer among the life-conditions of the species.

But if, at the end of a certain time, the factor B disappears from the education of the adult, the character, really and profoundly acquired, will not itself disappear on that account. It always manifests itself in the development of the embryo under the influence of conditions which are different from those which would make it function in the adult. Only, since it is no longer kept up by functional assimilation, it preserves at the end of embryo development the volume which it had acquired during this first portion of life and even begins a regression. But it is very difficult for it to disappear completely in the descent of the species because, since the conditions of embryo-development are altogether different from those of the functioning of the adult, the fact that the organ does not function in the adult will not prevent it from functioning for another reason in the embryo.

Thus the appendix of our coecum is developed in the intestine during our sojourn in our mother's womb, after which, not being used in our adult mechanism, it per- 
sists as a useless rudiment. But this disagreeable and dangerous rudiment may be long preserved in the species unless the epidemic of appendicitis, which has surprised the world for twenty years back, should be nothing other than the outcome of the introduction into our life-conditions of some unknown factor B which, after some generations, may end by unmaking in us that which in other days some other factor B had made.

\section{The Denial of the Heredity of Acquired Characters}

One thing may surprise us after all these considerations. In sum, they show us that strictly speaking there is never any hereditary transmission except of acquired characters. Yet a whole school of naturalists for several years has tried to deny the possibility of such transmission. The denial is the logical consequence of blind acceptance of the fanciful system of Weismann, in which the characters of an individual, instead of being considered as actual elements of a state of equilibrium, are taken to be static entities with an independent existence.

The Neo-Darwinian school has thus been led to consider that, in nature, no new characters ever make their appearance, but that there is only a re-arrangement and juxtaposition in variable order of characters which have existed from the dawn of life. It is enough to recall the fanciful defining of the characters of individuals for us to guess what the scientific value of such a system really is.

This wilful belief in static entities which do not and cannot exist is enough to condemn beforehand a theory which, moreover, obliges us to attribute a providential value to pure chance.

It is cited here only by way of mention, although it is still almost universally adopted-which may be explained 


\section{THE NATURE AND ORIGIN OF LIFE}

by the little knowledge of the exact sciences generally possessed by professional biologists. On the contrary, explanations based on equilibrium are very satisfying when we study the adaptive evolution of living species. A few pages will show this presently; but we must first occupy ourselves with a question that complicates in most cases that of heredity so-called. I mean the sexual phenomena so widespread in the animal and vegetable kingdoms. 


\section{PART IX}

Bipolarity in Living and Not-Living Matter 



\section{CHAPTER XXXII}

\section{SEXUALITY}

ONE of the oldest known facts of natural history is the necessity - at least, among the higher animals - of the intervention of two progenitors for the production of a new individual. Rabbit, crow, or lizard, though living and endowed with assimilation, can never reproduce itself when alone. In other words, there does not exist in these animals one single histological element which, separated from the parent body, can assimilate in the surrounding medium and build up, in virtue of the morpho-biological theorem, a new being like to the preceding.

In such beings the elements called reproductive or genital are impotent of themselves, incapable of assimilation. But there exist in every higher species two types generally very distinct externally, man and woman, stag and doe, cock and hen-in one word, male and female, such that the genital element of the male is the complement of that of the female. The fusion of these two elements of opposite sex-elements either of which is incapable of assimilationgives an egg capable of assimilation, the starting point of a new individual. And this fusion, which is the essential factor in fecundity, is facilitated by the very general fact that the female element attracts to itself the male element.

In animals of lower organization like the snail, leech, earth worm, the same individual produces at the same time 
incomplete elements of the two complementary types. Such an individual is called hermaphrodite and the notion of individual sex, which arises from the study of the higher animals, gives way to the notion of histological or genital sex. There are no longer a male and a female animal, but male and female reproductive elements produced by one and the same animal.

The further our investigations go the more we verify the generality of the sexual process of reproduction. A prudent biologist nowadays would never dare to assert that there is a single animal or vegetable species in which the sexual processus is wanting. Now such a generality of sexual processus makes us think that it must have some relation with a fundamental particularity of living substance.

There are, however, many cases in which we see the multiplication of an isolated cell coming from a cellular agglomeration that has to be considered as a perfect individual. But even where such parthenogenesis is possible, ${ }^{1}$ we generally see the sexual processus properly so called put in an appearance from time to time. An example is found in certain plant-lice which, throughout the fair season, reproduce by parthenogenesis and, when cold weather comes, take up again normal sexual reproduction.

The possibility of the two modes of reproduction, sexual and parthenogenetic, along with the extraordinary generality of the sexual processus, naturally gives rise to the idea that sexuality - a fundamental particularity of living substancemust exist in all vital acts, while manifesting itself only in certain cases in the form of cellular sexuality.

In other words, the notion of sexuality, first derived from observing the higher animals and then from the study of the reproductive elements of living beings, might be trans-

1 Parthenogenesis, from the Greek for virginal conception. 
ported to a third scale still smaller than the cellular scale : there might be intraprotoplasmic sexual phenomena, that is, intraprotoplasmic phenomena in which the intervention of two distinct and complementary elements would be indispensable to assimilation.

Remark at once that we already have an example of the necessity of two intracellular elements for the elementary life manifested by protozoa. Experiments in merotomy (Chap. XXVII) show that assimilation is produced neither in a cytoplasm deprived of nucleus nor in a nucleus without cytoplasm.

Suppose that, in certain species of protozoa, there should take place from time to time phenomena such that one cell would be reduced to its cytoplasm and another to its nucleus. Here we should have the two incomplete and complementary elements which would be called sexual. The hypothesis, moreover, is not beyond verification. Max Verworn has succeeded in extracting the nucleus from certain large marine Radiolaria and transplanting them into the cytoplasm of other individuals of the same species, thus realizing the equivalent of sexual maturation and fecundation. This experiment of Verworn reminds us of an electric pile, from which the zinc would be taken; it would no longer be an electric pile, for it is that only by the simultaneous presence of the two positive and negative poles. In other words, the pile is a bipolar apparatus; and so is the living cell since it needs a cytoplasm and a nucleus.

From the fact that the necessity of cytoplasm and nucleus proves clearly cellular bipolarity, it does not follow that cell elements cannot become incomplete otherwise than by the separation of cytoplasm and nucleus. The electric activity of a pile might be suspended by suppressing, not the zinc, but either the acidulated water or the conducting 


\section{THE NATURE AND ORIGIN OF LIFE}

wire; and yet you would separate the pile in two parts, of which the reunion would be indispensable to its functioning. In the same way, when the strange phenomenon of sexual maturation transforms the repro-

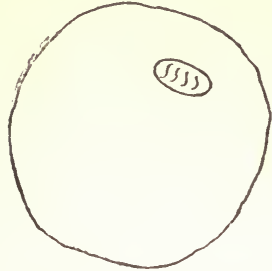

Fig. 16.-NATURe Ovule. ductive elements into incomplete cells, it is not by destroying the nucleus of the female and the cytoplasm of the male. At least, things pass otherwise morphologically.

There remains in the ovule or mature female element a body called nucleus, or more accurately female pronucleus (Fig. 16), drowned in a very considerable mass called ovulary cytoplasm.

In the same way, in the spermatozoid or mature male element there remains, beside the nucleus or male pronucleus, a very small quantity of substance often called male cytoplasm, or spermocentre (Fig. 17). Thus maturation is a less simple phenomenon, at least from the morphological side, than would be the separation of one single nucleus and one single cytoplasm. We have to resign ourselves to the idea that, as with many very important phenomena of biology, maturation is not susceptible of direct optical study because it

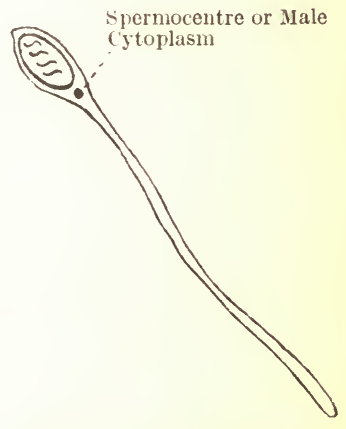

Fig. 17.-A Spermatozoid. does not consist in a production or destruction of figured elements of the cell. There is a nuclear maturation and a cytoplasmic maturation, thąt is, maturation transforms a complete nucleus into an incomplete pronucleus, male or female, and a complete cytoplasm into an incom- 
plete cytoplasm, male or female. Consequently, in order that the male or female maturation can be produced in the nucleus or the cytoplasm, it is necessary that the nucleus as well as the cytoplasm of a complete cell should contain the two sexes. Then maturation is explained by the disappearance in cytoplasm and nucleus of all elements of the sex opposed to that of the mature element finally obtained.

This being the case, without making any hypothesis as to the nature of the elements of opposite sex existing in either nuclear or cytoplasmic protoplasm, and without asking if these elements are chemical or colloid, we can represent the plan of a complete cell as an agglomeration of bipolar elements (Fig. 18, A). Male maturation should
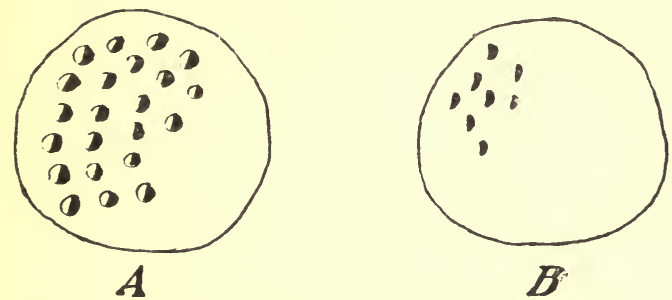

Fig. 18.-Diagram of Sexual Maturation.

be due to the disappearance of all the female half-elements and female maturation to the disappearance of all the male half-elements (Fig. 18, B).

What is the cause of maturation, male or female? We shall know only when we have discovered the nature itself of the differences which exist, in each bipolar element of protoplasm, between the male pole and the female pole.

\section{The Artificial Parthenogenesis of J. Loeb}

Meanwhile, without having discovered as much as this, J. Loeb has found an experimental means of suspending the 
maturation begun in the ovules of a sea urchin or star fish. For this he uses different processes, of which the earliest was the temporary immersion of the ovule under maturation in certain saline solutions. After the immersion, the ovule which was we know not how under way of maturing, showed an arrest in the phenomena of maturation begun within it and so found itself a complete cell and consequently capable of developing itself without fecundation by a spermatozoid. Probably, by such experiments, we may some day discover the very nature of phenomena of maturation.

Nature affords us examples of incomplete maturation. The bee's egg always remains in an intermediate stage between the complete cell formed solely of bipolar elements (Fig. 18, A) and the real female ovule formed solely of female poles (Fig. 18, B). The egg of the bee would be represented by a juxtaposition of bipolar elements and of elements reduced to their female pole. Consequently, such an egg is capable either of developing by itself or of being fecundated by a spermatozoid.

\section{Fecundation}

Fecundation is the operation in which the spermatozoid, introduced by sexual attraction into the ovule, completes by means of its male poles the female poles of the ovule's elements, which are incomplete.

While the optical study of mature sexual elements gives us no information about the nature itself of maturation, the observation of fecundation is, on the contrary, full of instruction inasmuch as it shows us with evidence various sexual attractions between corresponding parts of the elements that enter into fusion. Thus the male pronucleus is attracted toward the female pronucleus (Fig. 19) and directs itself toward it by pivoting around the male cytoplasm, 
which is adherent to it. During this time the male cytoplasm, localized in a very small region called the spermo-centre (Fig. 17), exercises its attraction on all the corresponding elements of the female cytoplasm, tracing in its substance radiating streaks which produce the figure called aster.

The movement of the male pronucleus toward the female pronucleus, on the one hand, and the formation of the cytoplasmic aster around the spermo-centre, on the other, show there is reciprocal attraction of the pronuclei among themselves and of the cytoplasms among themselves. There is, therefore a nucleary fecundation and a cytoplasmic fecundation, which proves that the diagram in Fig. 18 is
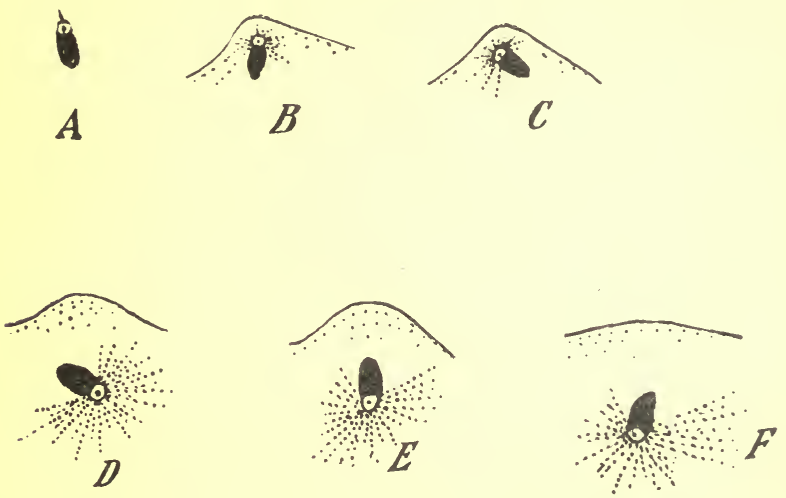

Fig. 19.-Sea Urchin's Spermatozoid entering Ovule.

exact, for the two fecundations show that maturation is at once intranuclear and intra-cytoplasmic, or, in one word, intraprotoplasmic.

We need not insist further on figured phenomena of fecundation. From our point of view their interest consists in showing with evidence that bipolarity is a phenomenon of smaller order than cellular magnitude. In other words, 
the ultimate sexual elements are smaller than cells; protoplasm is made up of particles, concerning the nature of which we make no hypothesis, but they have the two sexes. The two poles of these particles must coexist in the act of assimilation: assimilation is a bipolar phenomenon.

Here we have a result of prime importance with relation to the nature of vital phenomena.

Sex does not manifest itself to us in a manner easy to follow optically, except in the case where the phenomenon of sexual maturation (still unknown to us in its essence) reaches entirely the complete cellular elements so as to make of them sexual elements. In this case, in fact, we verify that there is cellular sex properly so-called-a cellular sex resulting, as we have just seen, from the total disappearance of one of the intracellular sexes in each of the intraprotoplasmic bipolar elements.

Thus, even in sexual phenomena, the ultimate element is not the cell, but is smaller than the cell. We came to this same conclusion for all ultimate elements of elementary life when we studied the Lamarckian or auto-adaptive variations of unicellular beings. On this account it is irrational to divide up a being into cells in the study of heredity as we have seen Darwin and Weismann doing.

Two phenomena, as general as they are mysterious, throw singular light on this notion of intraprotoplasmic bipolarity. They are alternating generation and karyokinesis. 


\section{CHAPTER XXXIII}

\section{ALTERNATING GENERATION}

As we have previously observed, we cannot define analytically the colloid state of living substances; and yet we can verify the importance of variations in this colloid state, particularly in histological differentiation. In every vertebrate animal, for example, we have for protoplasm the muscle state, the nerve state, the bone state, the epithelium state: these various tissue states correspond for so large a group as the vertebrates, and their number is considerable.

We never observe sexual maturation in these differentiated protoplasms, but we are obliged to say that, since bipolarity effectively exists in all the constituent elements of these various protoplasms, there must be in each type of the colloid state variations corresponding to modifications in bipolarity. An explanation may be taken from a hypothetical concrete example.

The two poles of one and the same bipolar element attract each other; but they are likewise in relation of attraction, or at least of cohesion, with the neighbouring elements. I suppose two states of equilibrium to be possible: one in which the two poles of each bipolar element touch, having come by attraction into contact with each other ; the other in which, under the influence of surrounding actions, the two poles of each bipolar element remain separated from each other as if by a tense spring. We may call one of 
them the associated, the other the dissociated state. It seems likely that, as well from the morphogenic as from other points of view, two such states will differ profoundly.

It must also be remarked that, according to the conditions, these two states may be derived hereditarily one from the other. In fact, it would be enough that any cause whatever should loosen the spring (cohesion, etc.) which kept apart the two poles of each bipolar element; and, because of the different morphogenic properties of the two states, we should have a being $B$ very different from a being $\mathrm{A}$ and yet coming from it by direct heredity.

Now such a phenomenon, precisely, is very general and the two states which we have imagined-the associated and the dissociated state-if they do not give us the real explanation, at least furnish a possible model of the phenomenon of alternating generation.

Alternating generation is found in its simplest form in ferns.

A fern with leaves, such as we are accustomed to finding along the wayside when autumn comes, has under its leaves little brownish grains of living substance in a state of latent life. 'They are spores or reproductive elements which have not undergone sexual maturation. But, while they have not undergone such maturation, they have at least experienced a modification in their state ; for they germinate and produce, not a leafy fern like that which gave birth to them, but a formless cellular mass resembling a green seaweed and called prothallus.

In the prothallus later on there appear sexual elements properly so-called, male and female, whose union gives eggs which reproduce the leafy fern. This closes the cycle of an obligatory alternating generation.

It is plain that such alternating generation is related with 
the bipolarity of living elements, since one of its forms-the prothallus-is always derived from an asexual reproduction, while the other-the fern-is always derived from sexual reproduction. Our provisional hypothesis enables us to class these two forms-fern and prothallus-in the two states which we have defined as associated and dissociated.

Besides this, there is a character which distinguishes the state of the prothallus from the state of the fern. It is that all karyokineses, or cellular divisions, are produced in the fern along with the appearance of $2 n$ chromosomes, whereas in the prothallus they are produced with an appearance of $n$ chromosomes only. (In the following chapter we shall see what chromosomes and karyokinesis are, but the logical order of thought leads us to place first the history of alternating generation.) Accordingly, we can substitute for prothallus state the denomination state of $n$ chromosomes.

Thus defined, the law of the two states becomes general. In all animals a generation of $n$ chromosomes alternates with a generation of $2 n$ chromosomes ; but, for the most part, the generation of $n$ chromosomes is included in the other as a parasite. This generation of $n$ chromosomes, or prothallus generation, constitutes the genital organs of the species under consideration. It is solely in the generation of $n$ chromosomes that sexual maturation manifests itselfwhich proves once again that the two successive states of living substance in alternating generation are related to the bipolarity of the living element. Until something new is known, we may admit-at least as a model for the fixing of our ideas-the hypothesis that the prothallus state cor responds with the associated state of bipolar elements, while the state of $2 n$ chromosomes answers to the dissociated state of these same elements. 


\section{CHAPTER XXXIV}

\section{KARYOKINESIS, OR INDIRECT CELLULAR DIVISION}

The progress of histological investigation has brought about the discovery, in cell division, of morphological manifestations which are a further proof in favour of intraprotoplasmic bipolarity. Professor Angel Gallardo, of Buenos Ayres, was the first to propose a bipolar theory of the karyokinetic phenomenon.

In karyokinesis there are nucleary manifestations and cytoplasmic manifestations.

The former have chiefly attracted the attention of students. They principally consist in a modification of the distribution of intranuclear chromatic substances, that is, of the parts of the nuclear protoplasm which are most avid of basic aniline colours. These chromatic substances, which are at first distributed in the form of an irregular network (Fig. 20, A), take the form of a long winding filament, or spireme (Fig. 20, B). Next, this winding filament is cut into a fixed nu mber of pieces, or chromosomes $(n$ in the prothallus state, $2 n$ in the fern state), each of which divides longitundinally into two parallel bead-strings. To these chromosomes has been attributed particular value in phenomena of the hereditary transmission of characters; but this is an hypothesis which nothing verifies.

In any case, this new distribution of the chromatic substance shows profound transformation in the equilibrium of 
the nuclear protoplasms ; and this transformation is further manifested by the disappearance of the nucleus membrane, that is, of the surface separating the nucleus from the cytoplasm. From this moment the substances of the nucleus are, so far as their equilibrium goes, subjected to certain actions coming from the cytoplasm. It is precisely these
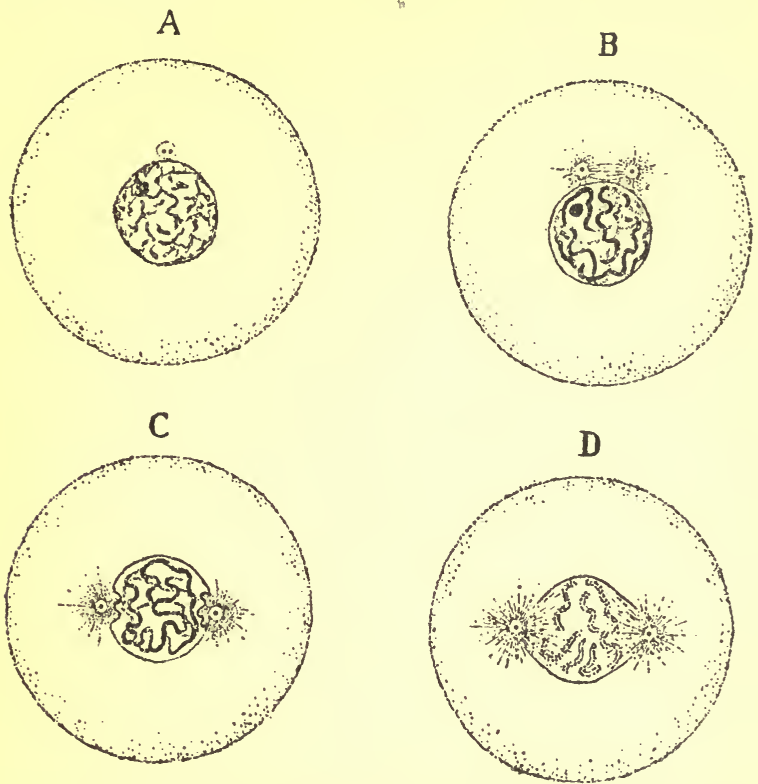

Fig. 20.-First Phenomena of Karyokinesis (from Wilson).

cytoplasmic actions which present an interesting bipolar character.

At the beginning of karyokinesis there appears in the cytoplasm, close to the surface of the nucleus, a little granule surrounded by a light aureole; this granule is called centrosome and almost at once divides in two (Fig. 20, A). Then the two centrosomes separate one from another, following the outer surface of the nucleus (at this stage the sur- 
face has not yet disappeared) and surround themselves with an aster identical with that which we saw appear around the spermo-centre in fecundation. These two centrosomes surrounded by their asters stop at diametrically opposite points of the nucleus (Fig. 20, D) and thenceforward the nucleus membrane disappears while the two centrosomes become centres of attraction for all the intraprotoplasmic substances, cytoplasmic or nuclear.
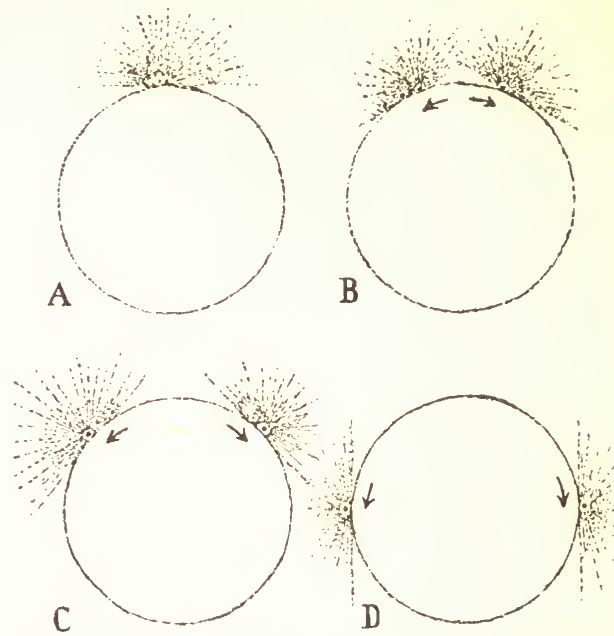

Fig. 21.-Diagram of Movement of Centrosomes around Nucleus in Karyokinesis.

They also become the starting point of two new cells, constituted like the mother-cell by a series of phenomena whose description may be found in all treatises on the cell (especially in Wilson's excellent The Cell in Development and Inheritance, which is everywhere known).

The only point concerning our study of the nature of living substance is this new demonstration of intraprotoplasmic bipolarity drawn from observations on centrosomes and 
asters. They form a true case of intracellular sexuality. The centrosome is a sexual element which, we know not why, has undergone sexual maturation in one direction, while the cytoplasmic substances have undergone maturation in an opposite direction. The asters are the image of a true intraprotoplasmic fecundation.

The movement of the centrosomes to the surface of the nucleus (Fig. 20, from state A to state C) is especially interesting and demonstrative of the attractions figured by the asters. It is possible that the two centrosomes, which have the same name, repel each other; but if we attend to the maturation in contrary directions of the cytoplasm as a whole, it is evident that the resultant attractions must finally, with the nucleus as a screen, bring the two centrosomes to positions diametrically opposed with relation to the nucleus. (Fig. 21, representing the movement of centrosomes around the nucleus, offers a diagram of these attractions and the resultant movement.)

Karyokinesis is thus a new proof of intraprotoplasmic bipolarity, or sexuality. It is a result of incomplete maturation, or rather of two maturations in contrary directions which, localized in the same cell, are able to correct each other. On the contrary, when maturation in a given direction takes possession of the whole cell, it gives a genital element which can no longer be completed except by an element that has matured in a contrary direction: these are cases of total cellular sexuality studied two chapters back.

The study of the phenomena of partial or total sexuality has increased our knowledge of the nature of life by a new and very important concept-the bipolarity of the elementary vital phenomenon. We have now to see the consequences of sexual reproduction in questions of heredity. 


\section{CHAPTER XXXV}

\section{HEREDITARY PATRIMONIES IN FECUNDATION}

Is our eighth part we studied phenomena of heredity, supposing the children to have a single parent, from which they receive directly the hereditary patrimony. This is verified in cases of parthenogenesis and also in reproduction by spores (in the latter case, however, heredity is often masked, at least morphologically, by the passage from the fern state to the prothallus state).

Things are otherwise in the most frequent cases. The egg comes from the fusion of a spermatozoid and an ovule taken from two different parents who consequently have different hereditary patrimonies. Clearly, the idea we have of the formation of the new hereditary patrimony in the act of fecundation will depend on the idea we have first adopted concerning the nature of sexual maturation and then of the manner in which fecundation is effected in detail. Whatever theory we adopt, there is one thing common to all, namely, that what is common to father and mother is transmitted integrally to the child in the act of fecundation. Discussion arises only concerning characters which differ in the progenitors. For example, two beings of the same species and race always have children of the same species and race as themselves. As to the characters which differ in father and mother-individual characters-biological schools sustain contradictory theses. 
Neo-Darwinians, with Weismann, deny the heredity of acquired characters and find no other causes of variation than the mixing of sexes or amphimixis. Consequently they consider successive fecundations as bringing into the living world a series of fortuitous variations, among which natural selection chooses.

Neo-Lamarckians, with Herbert Spencer, on the contrary, consider that sexual reproduction makes chance variations disappear and, consequently, maintains the average type of the species. Observation seems to verify the theory of Herbert Spencer ; on the other hand, a moment's reflection is enough to understand that, in our way of conceiving sexual phenomena, amphimixis takes an evident part as guardian of the average type of the species. ${ }^{1}$ Consequently, the rôle of sexual reproduction in the formation of species appears to us as a simple regulator of adaptive evolution.

In our next part, however, we shall see that amphimixis may realize, in certain cases, the sudden variations which De Vries has so thoroughly studied under the name of mutations, also called sportive variations. But the part played by mutations in the formation of species does not seem to have the importance now attributed to it by the Neo-Darwinian school.

\section{Secondary Sexual Characters}

In this matter of sex, it is necessary to point out the notable differences existing between the male and female of certain animals in which sex is separated, like man and other mammals. Experiments in castration prove that the genital organ plays a morphogenic part in the production of such differences. But we must also admit that, while the male

1 See development of this in author's Traité de Biologie. 


\section{THE NATURE AND ORIGIN OF LIFE}

genital parasite ${ }^{1}$ has an influence on the body which it inhabits, the body, on its side, has an influence on the genital parasite by determining the sexual direction in which it matures. The question of the nature and determination of the body's sex is much disputed. We apply the term "secondary sexual characters" to all characters appearing in the body under the influence of the genital organ and made to disappear by castration, when this takes place at a sufficiently precocious stage.

1 I speak of a genital parasite on account of what has previously been said-that the genital organ may be compared with the prothallus. 


\section{PART $X$}

Formation of Species and Appearance of Life 


\section{CHAPTER XXXVI}

\section{CONTINUOUS AND DISCONTINUOUS EVOLUTION}

The necessary limitation of the body of living individuals causes the evolution of life on the earth's surface to be represented by a succession of fragmentary masses, instead of being the history of one continuous mass growing indefinitely.

One result of this fragmentation is the opening of a wider field to variation. When an individual is once developed its anatomy, in fact, is more or less fixed by its skeleton which limits narrowly possible morphological variations. The reproductive element, being separated from the body, reproduces in all liberty a new individual and new skeleton, while the hereditary patrimony which it carries is able to conform itself better to external conditions.

A certain number of animals show a similar phenomenon in their individual existence. Crabs and lobsters, for example, are subject to periodical moulting. These animals are enclosed in a very resistant calcified crust, which opposes itself to their growth as well as to any variations whatever. By the phenomenon of moulting they are relieved of the hard part of their skeleton and, thus reduced to soft substance, take the form of equilibrium which agrees with them. All variation, all modification is limited in such animals to this phenomenon of moulting.

From a certain point of view, we can compare the specific 
evolution that takes place in a lineage of individuals with the individual evolution of an eternal individual which, from time to time, should undergo a moulting yet more complete than that of these crustaceous animals and should periodically become a plastic mass capable of fabricating for itself an entirely new equilibrium.

Thus the evolution of a line of individuals inevitably receives, if not a definitely discontinuous character, at least the aspect of an ascent of steps, of which each individual represents one, according to the happy expression of Professor Giard.

Even when the transformations which touch the hereditary patrimony are entirely continuous, the difference between two individual adults following each other in the lineage must be "finite." Consequently, the question whether variation has been slow or sudden in the series of ancestral forms of present species loses its seeming character of precision. That which mathematically would have had an exact meaning if specific evolution had been continuous or discontinuous, is no longer in question, but only whether the discontinuities which fatally exist between two successive individuals-finite discontinuities, as we have seen -are small or great. As the words small or great have no absolute meaning the interest of the question is lost.

In the individual evolution of certain living beings the last moult is accompanied by so wonderful a transformation that the individual issues from it quite unrecognizable. From a caterpillar it becomes a butterfly-surely a noteworthy variation. For this the name of metamorphosis has been reserved. Such a phenomenon is easily explained when we think how the genital organ, a morphogenic parasite, evolves at the same time as the host within which it is lodged and that a transformation in the state 
of this parasite (sexual maturation, for example) may show itself in a very important transformation of its host if the total transformation of the genital parasite has taken place in the interval between two moults. This probably happens to the species said to have complete metamorphosis, such as butterflies and beetles.

On the contrary, with other insects like grasshoppers, the transformation of the genital parasite is effected in the duration itself of several successive moults, at each of which a relatively slight modification is produced in the host. Thus the series of forms of the grasshopper, while it has the character of an ascent of steps, has a more continuous aspect than the corresponding series for the butterfly. It is as if you substituted several steps for the sudden leap from caterpillar to winged butterfly. We should not, however, be right in saying that evolution is continuous in the grasshopper and discontinuous in the butterfly. It is discontinuous in both cases, but in the first case a series of small discontinuities takes the place of the enormous discontinuity of the second.

Yet another reason introduces between individual and individual a stronger discontinuity than that which results from the fabrication of a new skeleton subjected to the rebound of variations acquired during the course of an individual existence. It is amphimixis.

Although we have no very certain knowledge of the manner in which the hereditary patrimony of the egg is constituted at the expense of the hereditary patrimonies of the two parents, yet we can see with the utmost evidence that in the phenomenon of fecundation there is a certain discontinuity. The egg is something new. It has an hereditary patrimony which is neither that of the father nor that of the mother, which constitutes itself in an instant 
at the moment of fecundation and introduces into the series of hereditary patrimonies an inevitable discontinuity. The only thing open to dispute relatively to a lineage propagated by sexual generation is not the continuity or discontinuity of its variations-a question without meaningbut the importance of the discontinuities produced at each generation.

\section{Mutations}

For some years great noise has been made about a discovery of De Vries which, according to many authors, would bring into question all Transformism. According to it, there would be a sudden appearance of new species. Instead of verifying that patient adaptation which Lamarck placed in the foremost rank of modes of variation, we should see appear from time to time, without known reason, new species capable henceforth of reproducing themselves indefinitely and differing by very clear-cut characters from the parent species whence they are derived.

In a bed of several thousand Oenothera plants there are found, for example, three or four individuals differing notably enough from the primitive species to merit a new denomination; and these individuals, kept apart, reproduce themselves in their own likeness. Here then, say the Neo-Darwinians, we have a new species obtained per saltum by sudden variation or mutation. It is well to reflect a little before we accept, on the faith of such observation, that all new species have thus appeared suddenly and by chance.

In the first place, what is a species? By what do we recognize that we have the right to call new species these aberrant types of Oenothera which chance to exist among the thousands of plants sowed by De Vries? We shall 
presently see how difficult it is to come to an agreement as to the definition of species.

On the other hand, the chances of amphimixis or fecundation are so great that, out of a number as large as you please of children coming from the same couple, there are never two identical. What is there astonishing in the fact that, out of the thousands of discontinuities separating the individuals coming from a sowing of seed, some should be greater than others? Such discontinuities should be still more striking when they are accompanied by a difference in colloid state, like that which separates muscle from nerve or, perhaps, the fern from the prothallus. It is enough to suppose that the specific protoplasm of Oenothera has a certain number of physical states equally stable to conceive how, influenced by the chances of amphimixis, certain plants are led to jump the steps which separate one of these states from the neighbouring state. As A. Giard has remarked in his Discourse at the St. Louis Exposition, it is possible that the following is what has happened. The protoplasms of the parents were grazing the step which separates two stable physical states, without actually landing at it. Then a chance variation due to amphimixis, without being actually greater than others, acquired special morphogenic importance by passing the bound which separated the two states.

Finally, yet another hypothesis is possible. More and more frequently, particularly in the vegetable kingdom, we find associations or symbioses between different species. Lichens result from the association of seaweed and mushroom; orchids are complete only when, at the beginning of their evolution, they are associated with mushrooms of the genus Fusarium. Possibly in many other plants there exist symbiotic microbes, too minute perhaps to 
be easily discovered under the microscope, and yet the sudden disappearance or introduction of one of these microbes in an individual might determine a considerable and hereditary morphological variation. In this hypothesis a mutation would not be the sudden appearance of a species, but the formation or destruction of a new association of two or more species already known.

In the present state of the question, we have no reason to adopt one explanation rather than another. The explanation by parasites is related with a very frequent phenomenon in the vegetable world-the formation of galls, of which the best-known is that of the oak or nutgall.

A gall is a local deformity of the plant, produced by the local action of an animal or vegetable parasite (in the case of the oak the nut-gall is produced by the development of eggs laid by an insect). This local deformity has great importance in biology, as may be seen from what has been said concerning morphogenic or formative diastases in connexion with the morpho-biological theorem. Here we see how one or more diastases emanating from the parasite are added, in the surrounding vegetable tissues, to the morphogenic diastase of such tissues and determine a new morphology in the infected region. The gall thus produced has a double specific quality, that is, it derives its characters at once from the species of the loost and the species of the parasite. Two different insects produce in the same plant two different galls. One and the same insect, when it is capable of developing in two different plants, also produces in these different plants different excrescences. There is here a whole chapter of biology, the descriptive part of which has even been given a special name-Cecidiology, or the description of galls. 


\section{CHAPTER XXXVII}

\section{THE SPECIES}

THE word species is in current use and its meaning seems clear enough not to need definition. We know the species man, horse, trout, viper, pear-tree ; and, so long as there is question only of such forms remote from each other, there is no difficulty. No one has any doubt in presence of such types. The difficulty begins with forms having great affinities with each other, such as the horse and ass, hare and rabbit, bulldog and poodle. Among such neighbouring forms we hesitate and essay an estimate of the differences which separate the two types, so as to come to a reasonable conclusion. In some cases the answer may be disconcerting to people not prepared for it. For example, the horse and ass are said to be of different species, while Dane and King Charles are ranked in the same species dog; and yet it is plain that the descriptive differences between the two dogs are far more considerable than those which we detect between the horse and the ass.

At bottom, under all this question about species, there lies an error of method.

For a very long time men had an idea that species were entities created separately and existing each on its own hook. In other words, it was believed that there was some absolute definition of species. The belief persisted even after the discovery of the transformation of species and 
men still feel the need of cataloguing, in strictly limited pigeon-holes, all these highly variable bodies called living beings. Evidently, such classification is indispensable in the narration of facts; but it is good enough for the purpose when it is clear and easy to handle. There is no necessity of asking whether our catalogue pigeon-holes are separated off by some principle of higher philosophy or some absolute definition of species already existing before men undertook to classify.

Yet even nowadays men ask what species is, instead of using their liberty to define the groups in which, for their own personal convenience, they shut up all living beings.

Cuvier's definition of species is still currently taught"The collection of all the organized beings descending from each other or from common parents and of those resembling them as much as they resemble each other."

This is a definition after the fact and not $\grave{a}$ priori, such as ought to be made when we have a catalogue classification on hand. In reality, it is not a definition at all, but a biological theorem and not strictly true at that. If we dissect Cuvier's proposition we find-first, our intuitive idea of beings of the same species; then a theorem that children are of the same species as their parents.

It goes without saying that Cuvier's definition, or rather the theorem hidden in it, is the denial of Transformism. If we accept in its strict sense the proposition- " children are of the same species as their parents"-we shall have to reason, step by step, to the necessary conclusion that the animals of the present day are of the same species as their remotest ancestors. Unluckily, people do not generally look so close in natural sciences and, in all courses of zoology, pupils are still taught together Transformism and Cuvier's definition ! 
To give absolute value to the idea of species, it would be necessary that the group called species should be separated from neighbouring groups by finite discontinuities; but it would also be necessary that there should exist perfect continuity among the different individual types constituting the species.

Now this is not the case.

We have seen how inevitable it is that finite differences should exist between any two living individuals. It is the existence of the step from any being whatever to every other being. Accordingly, when there is a step from one species to its neighbour, the discontinuity will differ only in dimension from that which separates two individuals of the same species.

The definition of species becomes a question of appreciation-it is no longer a strict and absolute definition.

Before Transformism, belief in the possibility of an absolute definition of species was possible and necessary. The step separating individuals of the same species might be passed over by the variations accompanying individual multiplication; but the discontinuities limiting species were impassable. This was the basis of an absolute definition-the variation resulting from adaptation and reproduction could not step over the limits of species. The species had, therefore, to be defined after the fact, as a result of prolonged observations. On the contrary, in the Transformist theory we are obliged to define species $\grave{a}$ prior $i$ for convenience in classification and without preoccupying ourselves with metaphysics.

I propose to define species-the sum-total of individuals among which only quantitative differences exist. Yet it is easy to see that this would be simply conventional and would even need other conventions, for we no longer believe 
in essential qualities. To say that two species differed qualitatively represents something which may be translated into quantitative language.

When De Vries showed us sudden mutations produced in Oenothera, many scholars gravely discussed the question whether the step passed over is a limit of species, whether, strictly speaking, we ought to consider the mutation to have passed over the limits of the species. There might have been sense in this before Transformism, but the definition of species has no longer any philosophical value.

We can take many points of view to estimate the discontinuity separating two different species. There are evidently as many as there are characters susceptible of separate description in the two species and of comparison as corresponding with each other. Now, as we have seen, the choice of such chsaacters is absolutely a matter of fancy.

Yet there is one phenomenon which enables us to compare at the same time all the characters of one species with c.ll the characters of another-fecundation. In this act we can put in presence of each other the hereditary patrimony of a male element of species A and the hereditary patrimony of a female element of species B. If fecundation ensues, it warrants us in defining a neighbourhood of the given species. Such a definition is worth more than one based on the consideration of some single character taken at random. Therefore the act of fecundation has always been chosen as a criterion by those who seek to give an absolute definition of species. 


\section{CHAPTER XXXVIII}

\section{GENEALOGY OF PRESENT-DAY SPECIES}

We shall pay no more attention to the definition of species. We ask one question only-how beings of the present day are derived from beings of other days. We know that the differences are great between the starting-point and the present; but we do not seek to find just when the passing of a step deserves to be called a change of species.

Beings of the present day are extremely numerous and most varied in form ; but there is nothing in their number or their variation to surprise us. We observe that not-living bodies are also very numerous and very varied. That which strikes us most in living beings is their extremely exact and co-ordinated mechanism, thanks to which each animal at each instant does exactly what it ought to do in order to continue living under the conditions in which it exists. ${ }^{1}$ In other words, an animal which has long lived in a certain environment is adapted to the environment.

Adaptation is that which the two great evolutionist schools set out to explain-both Lamarckian and Darwinian.

Lamarck undertook to explain adaptation directly and succeeded by means of the law of habit and the law of heredity of acquired characters. A defect of Lamarck's explanation was that, instead of considering en bloc the

1 I take an animal rather than a plant, because we find in animals the most wonderful co-ordinations. 
adaptation of individuals to circumstances, he decomposed the adaptation into factitious elements just as each of us decomposes his own actions by taking account of the psychological language with which we are familiar. In fact, Lamarck expressed adaptation by bringing in an intermediary stage of need. Such an animal, under such circumstances, experiences such needs, which determine the animal to act in such and such a way; and only for this second part of adaptation - the act resulting from the need -did he establish the law "the function creates the organ."

Darwin, not understanding the importance of Lamarck's work, imagined the language of natural selection after the fact. In Darwin's system all variations are fortuitous and not adaptive. This enables us to explain a certain number of things, especially in cases where the mechanism is not too complicated; and for this reason Darwinians oftenest choose their examples in the vegetable kingdom. With animals it is impossible to dispense with Lamarckian explanation; and I have already shown a bond of union which may be established between the Lamarckian and the Darwinian theory by applying the language of natural selection after the fact, not to animals nor even to the cells of animals, but to the smallest units susceptible of independent variations. Yet even this is but a subterfuge, and it would be better to take Lamarckism frankly as a result of the direct and reversible influence of the colloid mechanism on the chemical mechanism on one side, and on the anatomical mechanism on the other.

However this may be, by using now Lamarck's language and now Darwin's, we are able, not to recount in detail the progressive evolution which has led up to each present species, but at least not to suffer amazement in presence of the wonders of animal co-ordination. 


\section{Fritz Müller's Principle}

For the sake of recounting the origin of species, we shall not take up again the line of consideration followed out in recounting the individual evolution of a being. Our comparison between reproduction and a moult that suppresses the persistent skeleton is enough to show that the same language would be applicable. We can even transform our symbolic formulae by comprising the result of an entire individual life in a single equation and repeating for the transmission of acquired characters what we have said of the acquisition itself.

Let $a_{1}, a_{2}, \ldots a_{n}$ be the initial hereditary patrimonies of successive generations; and let $b_{1}, b_{2}, \ldots b_{n}$ be the total educations of each individual life. We can then represent by the symbolic formula $\left(a_{n} \times b_{n}\right)$ the whole vital functioning taken all together of the individual who is the $n$th term of the series; and we shall have this series of symbolic equations :

$$
\begin{aligned}
& a_{1}+\left(a_{1} \times b_{1}\right)=a_{2} \\
& a_{2}+\left(a_{2} \times b_{2}\right)=a_{3} \\
& a_{n-1}+\left(a_{n-1} \times b_{n-1}\right)=a_{n}
\end{aligned}
$$

The series of these symbolic equations represents specific evolution just as the corresponding series represented individual evolution.

A complication arises from the fact that, for the most part, each child is the issue of two parents; and so the hereditary patrimony of the $n$th generation will not be $a_{n}$, but a compromise between two different patrimonies belonging to two different individuals ; and this may be represented symbolically by

$$
\left(\frac{a_{n}+a^{\prime}}{2}{ }^{\prime}\right)
$$


In any case, whatever is common to $a_{n}$ and $a_{n}^{\prime}$ will be transmitted to the egg resulting from the union between the two parents and will inevitably comprise the characters acquired by the adaptation of individuals of the same species to identical conditions. Accordingly, with sufficient approximation we are able to neglect the complication of sexual reproduction in recounting the Lamarckian history of the progressive evolution of animals.

The series of symbolic equations of specific evolution has the same form as that of individual evolution. This is expressed, in figured language, by the principle of Fritz Müller :

"The embryology of an animal reproduces its genealogy." In other words, the series of individual characters appears in the same order as the series of corresponding ancestral characters. It is a fact of current observation that, when a character has appeared in the parent at a certain age, the same character appears in the child at a corresponding age, that is, after other characters whose pre-existence in the parent concurred in forming the given character. This simple remark comprises the entire principle of Fritz Müller.

There may, however, be a simplification in the series of embryonary forms. Instead of commencing at the stage $a_{1}$ it may begin at a later stage $a_{m}$ and will then comprise only the stages intermediary between $a_{m}$ and $a_{n}$. This takes place when, instead of leading a free life from the beginning as its ancestors did, the embryo passes the first stages of its existence inside an egg-shell or a matrix plentifully supplied with food material. Then it issues forth at the stage $a_{m}$, and the stages previous to $a_{m}$, in their conditions of parasitic life, take on a new form which is generally much simpler as mechanism than the free forms $a_{1}, a_{2}, \ldots$ $a_{r m}$. It is then said that there has been embroygenic fore- 
shortening. It is as if a crustacean should come out at the fourth or fifth moult instead of the first. The morpho-biological theorem enables us to understand this phenomenon, which proves once more that heredity is stronger than the education which, indeed, it directs within certain limits under pain of death. 


\section{CHAPTER XXXIX}

\section{APPEARANCE OF LIFE}

Succinct as they are, these previous considerations are sufficient for understanding the phenomenon of the progressive evolution of living organisms.

The study of the fossil forms discovered in geological strata-forms quite different from those of the present day-shows that animals of high organization appeared at comparatively recent dates. This gives us to understand that these higher species have been formed by a progressive accumulation of acquired characters resulting from individual adaptations to conditions that varied unceasingly. If we possessed the complete collection of ancestors of some present-day individual, we should find in them the history of the formation of its species and of all the adventures through which the species passed without ever dying. For the capital point of the evolutionary history of a species as much as that of an individual is that the evolution has never been interrupted by death. This enabled Darwin to assert that beings of the present day are an élite, and so on back from each preceding generation, since all their ancestors lived at least to the age of reproduction.

Just as the living being comes from an egg which, anatomically, is far more simple, so species of the present come from ancestral species of the past which, from every point of view, may have been enormously less complicated. Thus 
the Transformist theory, accepted as it is without dispute by men of science, goes back, not to that assuredly marvellous phenomenon which was the appearance of man on the earth's surface, but to the infinitely simpler phenomenon of the appearance of a living protoplasm reduced to the minimum sum total of hereditary characters.

Archimedes said, in a symbolic proposition which taken literally is absurd: "Give me a support for a lever and I will move the world." Just so the Transformist of to-day has the right to say: "Give me a living protoplasm and I will re-make the whole animal and vegetable kingdoms."

Of course, this is only a manner of speaking, for the remaking of animals and plants as they exist now would require not only the initial protoplasm, but also all the changes of circumstances which have so enriched it with varied and multiple heredity, that is, the evolution of the world itself ever since the appearance of life. For the evolution of life is bound up indissolubly with the evolution of the whole world.

The philosophic interest attaching to the question of the appearance of life on the earth's surface is easily understood. Moreover, the conditions of heat required for the existence of life prove that life did appear, for there was a time when temperature was too high for life to exist on the earth's surface. Lord Kelvin has made the supposition that a living cell may have been transported hither by a meteorite, but this only displaces the problem. All astronomic bodies have had an evolution analogous to that of the earth and life had always to make a first appearance. Consequently, it is simpler to accept the fact that it appeared on the earth's surface under circumstances which men of science are endeavouring to reproduce in their laboratories.

It is often said that Pasteur demonstrated the uselessness 
of such efforts. It is a mistake. Pasteur showed only this : By taking certain precautions we can keep from all invasion on the part of living species actually existing certain substances which might serve them as food. And that is all.

The problem of protoplasm synthesis remains what it was.

Our acquaintance with colloids is still so recent and rudimentary that we ought not to count on any speedy success in the efforts to fabricate a living cell. But the time will come when methodic analysis will allow of a reasoned synthesis. We have seen that scholars already begin to know how to decompose the activity of the living substance into transportable elements called diastases. It is probably along this way that we shall find the solution of the problem.

Perhaps, too, the solution will be hit on by chance. The learned world of to-day is so prepared for the discovery, that a premature announcement of spontaneous generation realized in gelatine subjected to the action of radium surprised nobody. When the effective synthesis is obtained, it will have no surprises in it-and it will be utterly useless. With the new knowledge acquired by science, the enlightened mind no longer needs to see the fabrication of protoplasm in order to be convinced of the absence of all essential difference and all absolute discontinuity between living and not-living matter. 


\title{
THE NEW KNOWLEDGE
}

\section{By PROFESSOR ROBERT KENNEDY DUNCAN.}

\author{
Illustrated. 8vo, Cloth, 6s. net.
}

"Professor Duncan has written a most lucid and interesting book for those who wish to know something of the birth of matter, the decay of matter, the nature of matter, of the nature of electricity and the relation of electricity to matter, of the nature of the sun and the sun's rays, of the possible cause of gravitation, the cause of clouds and rain, and the reasonable solution of many another mystery. His book shows an admirable power of exposition... . we have as yet seen no book so well adapted to explain to the layman the meaning and the bearing of the latest researches into the mystery of the universe."-Spectator.

" The man in the street, by the time he has finished the two hundred and fifty pages of clear type here presented to him, will have a much more lucid idea of the recent advance in physics and the conclusions to which it has given rise than he can have at the present moment. The book is a great improvement upon all previous attempts at its popularization that we have seen. . . . The best of its kind we have read."-Athenoum.

"There is at the present no book on the márket with the same aim which gives the required information so well as does 'The New Knowledge.' . . . The book is really extremely well done, and a book of this kind was badly wanted. . . . The reader will rise from a perusal of these pages with a very good idea of radio-activity, and of the theories to which its discovery has given rise."-Westminster Gazette."

"Let nobody be discouraged by the title of 'The New Knowledge' which Robert Kennedy Duncan has given to a volume which sets out in language which is plain-spoken and easily understood a good many of the new views in chemistry and physics that the lately imagined anatomy of the atom has created. His volume is one which can confidently be recommended to that vast army of inquirers, who, not themselves being scientific students of physics, are yet possessed of trained intelligence, and who want a good book on the whole subject."-Knowledge.

"In a long experience of scientific exposition, both professional and amateur, we have come across few more daring efforts than that of Professor Duncan in the present volume. He has attempted a popular account of the new physics and the new chemistry in their relation to the new theory of matter. It was a bold venture. But Professor Duncan has succeeded, and told as much of the new knowledge and its potentiality on the coming thought as anv one not a professed scientist may desire to know.'.-Pall Mall Gazette.

\section{LONDON : HODDER AND STOUGHTON.}




\section{THE ASCENT OF MAN.}

BY PROFESSOR HENRY DRUMMOND.

Crown- 8vo, Cloth, 3s. 6d. net.

A SHORT HISTORY OF MEDIEVAL PEOPLES. By ROBINSON SOUTTAR, M.A., D.C.L.

Royal $8 v o$, i2s.

\section{A SHORT HISTORY OF ANCIENT PEOPLES.} BY THE SAME AUTHOR.

Royal 8vo, I2s.

RELICS OF PRIMEVAL LIFE.

BY SIR J. WILLIAM DAIISON.

Crown Svo, Cloth, 6s.

THE ORJGIN OF THE WORLD.

BY THE SAME AITHOR.

Crimen 8vo, Cloth, 7s. 6d.

THE STORI OF EARTH AND MAN.

BI THE SAME AUTHOR.

Crown 8vo, Cloth, 7s. 6a.

THE BRAIN AND PERSONALITY.

BY W. H. THOMSON, M.D., LL.D.

Crown 8vo, Cloth.

THE UNCONSCIOUS MIND.

BY A. T. SCHOFIELD, M.D.

Crown 8vo, Cloth, 7s. 6d.

LONDON : HODDER AND STOUGHTON. 


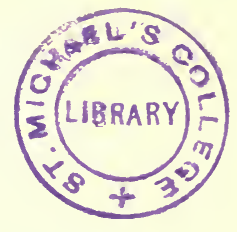




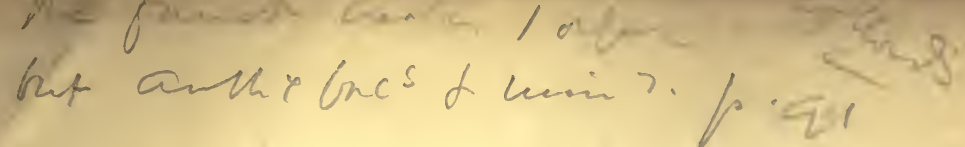


Gepee. Vitichocr

4. Cluncenatir. bur!

11. Sunbryoles

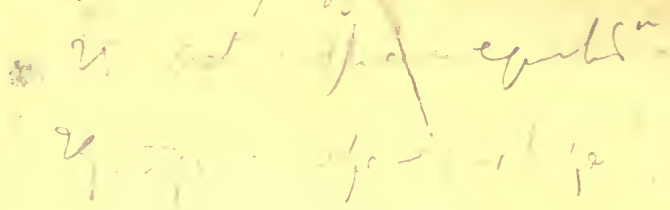

2,

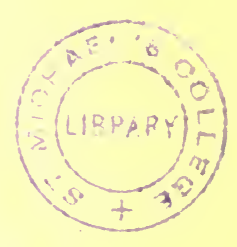

10

1

2

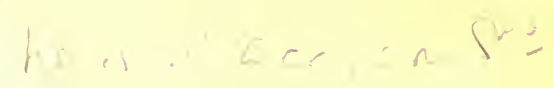


IE DANTEC, '́.A.

24

325

The nature and origin of life.I4 
